\title{
Ward identities and Chiral anomaly in the Luttinger liquid
}

\author{
G. Benfatto, V. Mastropietro \\ Dipartimento di Matematica, Università di Roma "Tor Vergata" \\ Via della Ricerca Scientifica, I-00133, Roma
}

\begin{abstract}
Systems of interacting non relativistic fermions in $d=1$, as well as spin chains or interacting bidimensional Ising models, verify an hidden approximate Gauge invariance which can be used to derive suitable Ward identities. Despite the presence of corrections and anomalies, such Ward identities can be implemented in a Renormalization Group approach and used to exploit nontrivial cancellations which allow to control the flow of the running coupling constants; in particular this is achieved combining Ward identities, Dyson equations and suitable correction identities for the extra terms appearing in the Ward identities, due to the presence of cutoffs breaking the local gauge symmetry. The correlations can be computed and show a Luttinger liquid behavior characterized by non universal critical indices, so that the general Luttinger liquid construction for one dimensional systems is completed without any use of exact solutions. The ultraviolet cutoff can be removed and a Quantum Field Theory corresponding to the Thirring model is also constructed.
\end{abstract}

\section{Introduction}

\subsection{Luttinger liquids.}

A key notion in solid state physics is the one of Fermi liquids, used to describe systems of interacting electrons which, in spite of the interaction, have a physical behavior qualitatively similar to the one of the free Fermi gas. In analogy with Fermi liquids, the notion of Luttinger liquids has been more recently introduced, to describe systems behaving qualitatively as the Luttinger model, see for instance $[\mathrm{A}]$ or $[\mathrm{Af}]$; their correlations have an anomalous behavior described in terms of non universal (i.e. nontrivial functions of the coupling) critical indices.

A large number of models, for which an exact solution is lacking (at least for the correlation functions) are indeed believed to be in the same class of universality of the Luttinger model or of its massive version, and indeed in the last decade, starting from [BG], it has been possible to substantiate this assumption on a large class of models, by a quantitative analysis based on Renormalization Group techniques, which at the end allow us to write the correlations and the critical indices as convergent series in the coupling. We mention the Schwinger functions of interacting non relativistic fermions in $d=1$ (modelling the electronic properties of metals so anisotropic to be considered as one dimensional), in the spinless [BGPS], in the spinning case with repulsive interaction $[\mathrm{BoM}]$, or with external periodic or quasi-periodic potentials $[\mathrm{M}]$; the spin-spin correlations of the Heisenberg $X Y Z$ spin chain [BM1], [BM2]; the thermodynamic functions of classical Ising systems on a bidimensional lattice with quartic interactions like the Eight-vertex or the Ashkin-Teller models [M1]; and many others, see for instance the review [GM].

In all such models the observables are written as Grassmannian integrals, and a naive evaluation of them in terms of a series expansion in the perturbative parameter does not work; it is however possible, by a multiscale analysis based on Renormalization Group, to write the Grassmannian integrals as series of suitable finitely many parameters, called running coupling constants, and this expansion is convergent if the running coupling constants are small. The running coupling constants obey a complicated set of recursive equations, whose right hand side is called, as usual, the Beta function. The Beta function can be written as sum of two terms; one, which we call principal part of the Beta function, is common to all such models while the other one is model dependent. It 
turns out that, if the principal part of the beta function is asymptotically vanishing, than the flow of the running coupling constants in all such models can be controlled just by dimensional bounds, and the expansion is really convergent; the observables are then expressed by explicit convergent series from which the physical information can be extracted. On the other hand the principal part of the Beta function coincides with the Beta function of a model, which we call reference model, describing two kind of fermions with a linear "relativistic" dispersion relation and with momenta restricted by infrared and ultraviolet cutoffs, interacting by a local quartic potential.

In order to prove the vanishing of the Beta function of the reference model (in the form of the bound (3.3) below), one can use an indirect argument, based on the fact that the reference model is close to the Luttinger model, and then use the Luttinger model exact solution of [ML], see [BGPS], [BM3].

In this paper we show that indeed the vanishing of the principal part of the Beta function can be proved without any use of the exact solution, by using only Ward identities based on the approximate chiral gauge invariance of the reference model.

As exact solutions are quite rare and generally peculiar to $d=1$, while RG analysis and Ward identities are general methods working in any dimension, our results might become relevant for the theory of non-Fermi liquids in $d>1$. We remember that there is experimental evidence for non Fermi liquid (probably Luttinger) behavior in high $T_{c}$ superconductors $[\mathrm{A}]$, which are essentially planar systems.

Ward identities play a crucial role in Quantum Field Theory and Statistical Mechanics, as they allow to prove cancellations in a non perturbative way. The advantage of reducing the analysis of quantum spin chains or interacting Ising models to the reference model is that such model, formally neglecting the cutoffs, verifies many symmetries which were not verified by the spin chain or spin models; in particular it verifies a total gauge invariance symmetry $\psi_{\mathbf{x}, \omega}^{ \pm} \rightarrow e^{ \pm i \alpha_{\mathbf{x}}} \psi_{\mathbf{x}, \omega}^{ \pm}$and chiral gauge invariance $\psi_{\mathbf{x}, \omega}^{ \pm} \rightarrow e^{ \pm i \alpha_{\mathbf{x}, \omega}} \psi_{\mathbf{x}, \omega}^{ \pm}$. Such symmetries are hidden in Ising or spin chain models, as they do not verify chiral gauge invariance, even if they are "close", in an RG sense, to a model formally verifying them.

\subsection{The reference model.}

The reference model is not Hamiltonian and is defined in terms of Grassmannian variables. It describes a system of two kinds of fermions with linear dispersion relation interacting with a local potential; the presence of an ultraviolet and an infrared cutoff makes the model not solvable. Given the interval $[0, L]$, the inverse temperature $\beta$ and the (large) integer $N$, we introduce in $\Lambda=[0, L] \times[0, \beta]$ a lattice $\Lambda_{N}$, whose sites are given by the space-time points $\mathbf{x}=\left(x, x_{0}\right)=$ $\left(n a, n_{0} a_{0}\right), a=L / N, a_{0}=\beta / N, n, n_{0}=0,1, \ldots, N-1$. We also consider the set $\mathcal{D}$ of space-time momenta $\mathbf{k}=\left(k, k_{0}\right)$, with $k=\frac{2 \pi}{L}\left(n+\frac{1}{2}\right)$ and $k_{0}=\frac{2 \pi}{\beta}\left(n_{0}+\frac{1}{2}\right), n, n_{0}=0,1, \ldots, N-1$. With each $\mathbf{k} \in \mathcal{D}$ we associate four Grassmannian variables $\hat{\psi}_{\mathbf{k}, \omega}^{[h, 0] \sigma}, \sigma, \omega \in\{+,-\}$. Then we define the functional integration $\int \mathcal{D} \psi^{[h, 0]}$ as the linear functional on the Grassmann algebra generated by the variables $\hat{\psi}_{\mathbf{k}, \omega}^{[h, 0] \sigma}$, such that, given a monomial $Q(\hat{\psi})$ in the variables $\hat{\psi}_{\mathbf{k}, \omega}^{[h, 0] \sigma}$, its value is 0 , except in the case $Q(\hat{\psi})=\prod_{\mathbf{k} \in \mathcal{D}, \omega= \pm} \hat{\psi}_{\mathbf{k}, \omega}^{[h, 0]-} \hat{\psi}_{\mathbf{k}, \omega}^{[h, 0]+}$, up to a permutation of the variables. In this case the value of the functional is determined, by using the anticommuting properties of the variables, by $\int \mathcal{D} \psi^{[h, 0]} Q(\hat{\psi})=1$.

The lattice $\Lambda_{N}$ is introduced only to allow us to perform a non formal treatment of the Grassmannian integrals, as the number of Grassmannian variables is finite, and eventually the limit $N \rightarrow \infty$ is taken, see [BM1].

We also define the Grassmannian field on the lattice $\Lambda_{N}$ as

$$
\psi_{\mathbf{x}, \omega}^{[h, 0] \sigma}=\frac{1}{L \beta} \sum_{\mathbf{k} \in \mathcal{D}} e^{i \sigma \mathbf{k x}} \hat{\psi}_{\mathbf{k}, \omega}^{[h, 0] \sigma}, \quad \mathbf{x} \in \Lambda_{N} .
$$

Note that $\psi_{\mathbf{x}, \omega}^{[h, 0] \sigma}$ is antiperiodic both in time and space variables.

$11 /$ ottobre/2018; $1: 16$ 
We define

$$
V\left(\psi^{[h, 0]}\right)=\lambda \int d \mathbf{x} \psi_{\mathbf{x},+}^{[h, 0]+} \psi_{\mathbf{x},+}^{[h, 0]-} \psi_{\mathbf{x},-}^{[h, 0]+} \psi_{\mathbf{x},-}^{[h, 0]-}
$$

and

$$
P\left(d \psi^{[h, 0]}\right)=\mathcal{N}^{-1} \mathcal{D} \psi^{[h, 0]} \cdot \exp \left\{-\frac{1}{L \beta} \sum_{\omega= \pm 1} \sum_{\mathbf{k} \in \mathcal{D}} C_{h, 0}(\mathbf{k})\left(-i k_{0}+\omega k\right) \hat{\psi}_{\mathbf{k}, \omega}^{[h, 0]+} \hat{\psi}_{\mathbf{k}, \omega}^{[h, 0]-}\right\}
$$

with $\mathcal{N}=\prod_{\mathbf{k} \in \mathcal{D}}\left[(L \beta)^{-2}\left(-k_{0}^{2}-k^{2}\right) C_{h, 0}(\mathbf{k})^{2}\right]$ and $\int d \mathbf{x}$ is a shorthand for " $a a_{0} \sum_{\mathbf{x} \in \Lambda_{N}}$ ". The function $C_{h, 0}(\mathbf{k})$ acts as an ultraviolet and infrared cutoff and it is defined in the following way. We introduce a positive number $\gamma>1$ and a positive function $\chi_{0}(t) \in C^{\infty}\left(\mathbb{R}_{+}\right)$such that

$$
\chi_{0}(t)= \begin{cases}1 & \text { if } 0 \leq t \leq 1 \\ 0 & \text { if } t \geq \gamma_{0}, \quad 1<\gamma_{0} \leq \gamma\end{cases}
$$

and we define, for any integer $j \leq 0, f_{j}(\mathbf{k})=\chi_{0}\left(\gamma^{-j}|\mathbf{k}|\right)-\chi_{0}\left(\gamma^{-j+1}|\mathbf{k}|\right)$. Finally we define $\chi_{h, 0}(\mathbf{k})=\left[C_{h, 0}(\mathbf{k})\right]^{-1}=\sum_{j=h}^{0} f_{j}(\mathbf{k})$ so that $\left[C_{h, 0}(\mathbf{k})\right]^{-1}$ is a smooth function with support in the interval $\left\{\gamma^{h-1} \leq|\mathbf{k}| \leq \gamma\right\}$, equal to 1 in the interval $\left\{\gamma^{h} \leq|\mathbf{k}| \leq 1\right\}$.

We call $\psi^{[h, 0]}$ simply $\psi$ and we introduce the generating functional

$$
\mathcal{W}(\phi, J)=\log \int P(d \psi) e^{-V(\psi)+\sum_{\omega} \int d \mathbf{x}\left[J_{\mathbf{x}, \omega} \psi_{\mathbf{x}, \omega}^{+} \psi_{\mathbf{x}, \omega}^{-}+\phi_{\mathbf{x}, \omega}^{+} \psi_{\mathbf{x}, \omega}^{-}+\psi_{\mathbf{x}, \omega}^{+} \phi_{\mathbf{x}, \omega}^{-}\right]} .
$$
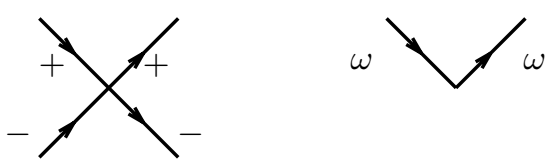

Fig. 1: Graphical representation of the interaction $V(\psi)$ and the density $\psi_{\mathbf{x}, \omega}^{+} \psi_{\mathbf{x}, \omega}^{-}$

The Grassmannian variables $\phi_{\mathbf{x}, \omega}^{\sigma}$ are antiperiodic in $x_{0}$ and $x$ and anticommuting with themselves and $\psi_{\mathbf{x}, \omega}^{\sigma}$, while the variables $J_{\mathbf{x}, \omega}$ are periodic and commuting with themselves and all the other variables. The Schwinger functions can be obtained by functional derivatives of (1.5); for instance

$$
\begin{gathered}
G_{\omega}^{2,1}(\mathbf{x} ; \mathbf{y}, \mathbf{z})=\left.\frac{\partial}{\partial J_{\mathbf{x}, \omega}} \frac{\partial^{2}}{\partial \phi_{\mathbf{y},+}^{+} \partial \phi_{\mathbf{z},+}^{-}} \mathcal{W}(\phi, J)\right|_{\phi=J=0}, \\
G_{\omega}^{4,1}\left(\mathbf{x} ; \mathbf{x}_{1}, \mathbf{x}_{2}, \mathbf{x}_{3}, \mathbf{x}_{4}\right)=\left.\frac{\partial}{\partial J_{\mathbf{x}, \omega}} \frac{\partial^{2}}{\partial \phi_{\mathbf{x}_{1}, \omega}^{+} \partial \phi_{\mathbf{x}_{2}, \omega}^{-}} \frac{\partial^{2}}{\partial \phi_{\mathbf{x}_{3},-\omega}^{+} \partial \phi_{\mathbf{x}_{4},-\omega}^{-}} \mathcal{W}(\phi, J)\right|_{\phi=J=0}, \\
G_{\omega}^{4}\left(\mathbf{x}_{1}, \mathbf{x}_{2}, \mathbf{x}_{3}, \mathbf{x}_{4}\right)=\left.\frac{\partial^{2}}{\partial \phi_{\mathbf{x}_{1}, \omega}^{+} \partial \phi_{\mathbf{x}_{2}, \omega}^{-}} \frac{\partial^{2}}{\partial \phi_{\mathbf{x}_{3},-\omega}^{+} \partial \phi_{\mathbf{x}_{4},-\omega}^{-}} \mathcal{W}(\phi, J)\right|_{\phi=J=0}, \\
G_{\omega}^{2}(\mathbf{y}, \mathbf{z})=\left.\frac{\partial^{2}}{\partial \phi_{\mathbf{y}, \omega}^{+} \partial \phi_{\mathbf{z}, \omega}^{-}} \mathcal{W}(\phi, J)\right|_{\phi=J=0} .
\end{gathered}
$$



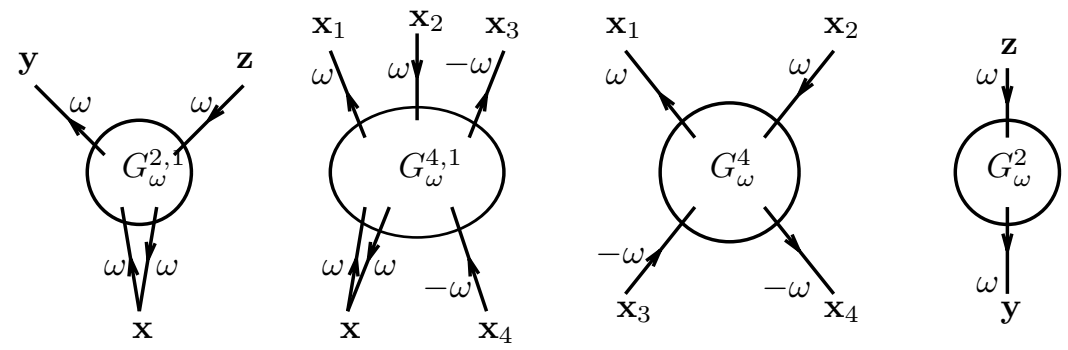

Fig. 2: Graphical representation of the Schwinger functions $G_{\omega}^{2,1}, G_{\omega}^{4,1}, G_{\omega}^{4}, G_{\omega}^{2}$

If $Q(\psi)$ is a monomial in the Grassmannian variables, it is easy to see that $\int P(d \psi)$ is given by the anticommutative Wick rule; the corresponding propagator

$$
\hat{g}_{\omega}(\mathbf{k})=\frac{\chi_{h, 0}(\mathbf{k})}{-i k_{0}+\omega k}
$$

is singular as $L, \beta \rightarrow \infty$ at $\mathbf{k}=0$. The simplest way of computing the Grassmannian integral (1.5) is to expand in power series of $\lambda$ the exponential, obtaining many Grassmannian integrals of monomials, which can be computed by the anticommutative Wick rule. This procedure makes it possible to write series expansions for the Schwinger functions, for example those defined in (1.6)-(1.9), and to prove that they are absolutely convergent, in the limit $L, \beta \rightarrow \infty$, for $|\lambda| \leq \varepsilon_{h}$, with $\varepsilon_{h} \rightarrow 0$ as $h \rightarrow-\infty$; in other words, the estimated radius of convergence vanishes as the infrared cutoff is removed. In this paper we will show that it is possible to modify the expansions, through a resummation of the power series in $\lambda$, so that it is possible to prove that they are well defined and convergent even when the infrared cutoff is removed $(h \rightarrow-\infty)$; as a corollary we prove vanishing of the Beta function in the form of the bound (3.3) below.

It is also possible to remove the ultraviolet cutoff, so constructing a relativistic quantum field theory, the massless Thirring model [T]; this is shown in the Appendix. Note finally that the reference model, if both the infrared and ultraviolet cutoffs are removed and the local potential is replaced by a short-ranged one, coincides with the Luttinger model, which was solved in [ML]; the presence of cutoffs makes however the model (1.5) not solvable.

\subsection{Sketch of the proof: the Dyson equation.}

This paper is the conclusion of our construction of $d=1$ Luttinger liquids with no use of exact solutions, started in [BM1], [BM2], [BM3], whose results will be used here.

The analysis starts by expressing the Grassmann integration in (1.5) as the product of many independent integrations, each of them "describing the theory at a certain momentum scale $\gamma^{j}$ ", with $j$ an integer such that $h \leq j \leq 0$ and $\gamma>1$. This allows us to perform the overall functional integration by iteratively integrating the Grassmanian variables of decreasing momentum scale. After $|j|$ integration steps one gets a Grassmannian integral still similar to (1.5), the main differences being that the Grassmannian fields acquire a wave function renormalization $Z_{j}$, the local terms quartic in the $\psi$ fields in the interaction have coupling $\lambda_{j}$ (the effective interaction strength at momentum scale $\gamma^{j}$ ) and the local terms $J \psi_{\omega}^{+} \psi_{\omega}^{-}$have coupling $Z_{j}^{(2)}$ (the density renormalization). This iterative procedure allows us to get an expansion, resumed in $\S 2$, for the Schwinger functions; they are written as series in the set of parameters $\lambda_{j}, j=0,-1, \ldots h$, called running coupling constants. It was proved in [BM1] that, if the running coupling constants are small enough, such expansions are convergent, as a consequence of suitable cancellations due to the anticommutativity of fermions.

However, the property that the running coupling constants are small is not trivial at all; it is related to very complex and intricate cancellations at all orders in the perturbative series, eventually 
implying that the effective interaction strength $\lambda_{j}$ stays close to its initial value $\lambda$ for any $j$; while one can easily check that cancellations are present at lowest orders by an explicit computation, to prove directly that the cancellations are present at every order seems essentially impossible. In order to prove that $\lambda_{j}$ remains close to $\lambda$ for any $j$, we use the fact that the Schwinger functions, even if they are expressed by apparently very different series expansion, are indeed related by remarkable identities; on the other hand the Fourier transform of Schwinger functions computed at the cut-off scale are related to the running coupling constants or to the renormalization constants at the cutoff scale (see Theorem 2.1), so that identities between Schwinger functions imply identities between coupling or renormalization constants.

As $\hat{G}^{4}$ computed at the cutoff scale is proportional to $\lambda_{h}$, see $(2.37)$ below, it is natural to write (see $\S 3$ ) a Dyson equation for $\hat{G}^{4}$ :

$$
\begin{aligned}
-\hat{G}_{+}^{4}\left(\mathbf{k}_{1}, \mathbf{k}_{2}, \mathbf{k}_{3}, \mathbf{k}_{4}\right) & =\lambda \hat{g}_{-}\left(\mathbf{k}_{4}\right)\left[\hat{G}_{-}^{2}\left(\mathbf{k}_{3}\right) \hat{G}_{+}^{2,1}\left(\mathbf{k}_{1}-\mathbf{k}_{2}, \mathbf{k}_{1}, \mathbf{k}_{2}\right)+\right. \\
& \left.+\frac{1}{L \beta} \sum_{\mathbf{p}} G_{+}^{4,1}\left(\mathbf{p} ; \mathbf{k}_{1}, \mathbf{k}_{2}, \mathbf{k}_{3}, \mathbf{k}_{4}-\mathbf{p}\right)\right]
\end{aligned}
$$

relating the correlations in (1.6),(1.7),(1.8),(1.9); see Fig. 3.

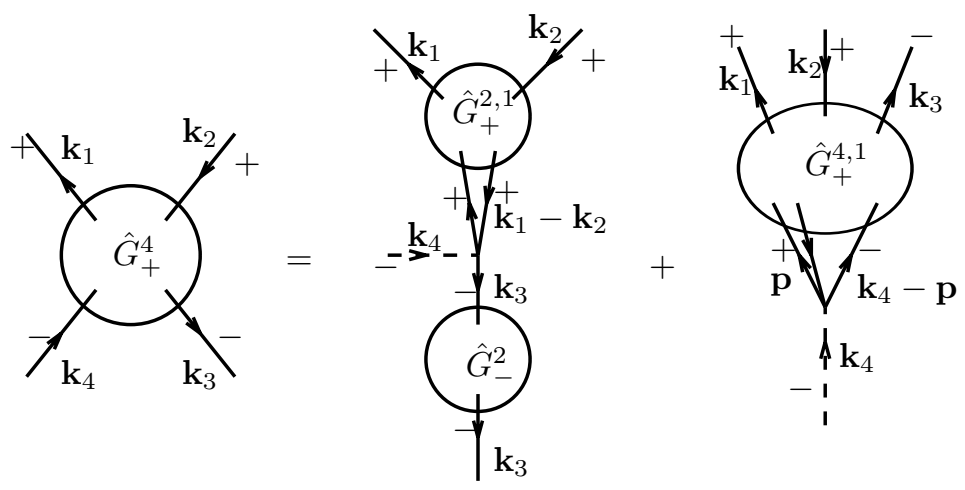

Fig. 3: Graphical representation of the Dyson equation (1.11); the dotted line represents the "bare" propagator $g\left(\mathbf{k}_{4}\right)$

The 1.h.s. of the Dyson equation computed at the cutoff scale is indeed proportional to the effective interaction $\lambda_{h}$ (see (2.37) below), while the r.h.s. is proportional to $\lambda$. If one does not take into account cancellations in (1.11), this equation only allows us to prove that $\left|\lambda_{h}\right| \leq C_{h}|\lambda|$, with $C_{h}$ diverging as $h \rightarrow-\infty$. However, inspired by the analysis in the physical literature, see [DL], [S], [MD], we can try to express $\hat{G}_{\omega}^{2,1}$ and $\hat{G}_{\omega}^{4,1}$, in the r.h.s. of (1.11), in terms of $\hat{G}_{\omega}^{2}$ and $\hat{G}_{\omega}^{4}$ by suitable Ward identities and correction identities.

\subsection{Ward identities and the first addend of (1.11).}

To begin with, we consider the first addend in the r.h.s. of the Dyson equation (1.11). A remarkable identity relating $\hat{G}_{+}^{2,1}$ to $\hat{G}_{+}^{2}$ can be obtained by the chiral Gauge transformation $\psi_{\mathbf{x},+}^{ \pm} \rightarrow$ $e^{ \pm i \alpha_{\mathbf{x}}} \psi_{\mathbf{x},+}^{ \pm}, \psi_{\mathbf{x},-}^{ \pm} \rightarrow \psi_{\mathbf{x},-}^{ \pm}$in the generating functional (1.5); one obtains the identity (3.14) below, represented pictorially in Fig. 4, with $D_{\omega}(\mathbf{p})=-i p_{0}+\omega p$. 


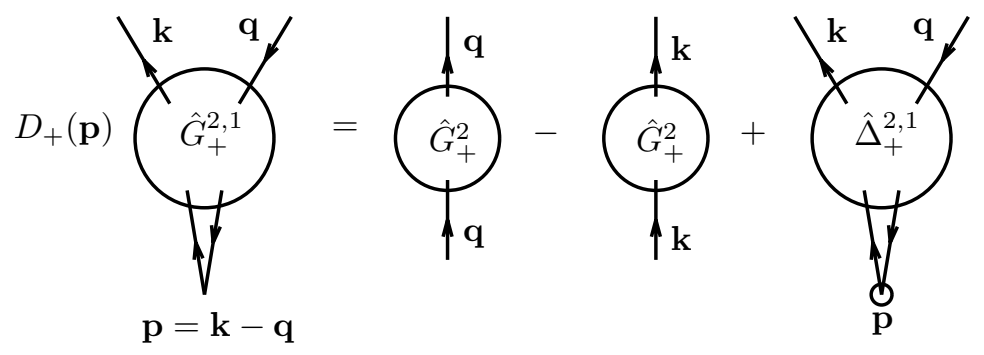

Fig. 4: Graphical representation of the Ward identity (3.14); the small circle in $\hat{\Delta}_{+}^{2,1}$ represents the function $C_{+}$of $(3.16)$

The above Ward identity provides a relation between $\hat{G}_{+}^{2,1}, \hat{G}_{+}^{2}$ and a correction term $\hat{\Delta}_{+}^{2,1}$, which can be obtained, through the analogue of (1.6), from a functional integral very similar to (1.5), with the difference that $\sum_{\omega} \int d \mathbf{p} d \mathbf{k} \hat{J}_{\mathbf{p}, \omega} \psi_{\mathbf{k}, \omega}^{+} \psi_{\mathbf{k}-\mathbf{p}, \omega}^{-}$is replaced by $\int d \mathbf{p} d \mathbf{k} C_{+}(\mathbf{k}, \mathbf{k}-\mathbf{p}) \hat{J}_{\mathbf{p}} \psi_{\mathbf{k},+}^{+} \psi_{\mathbf{k}-\mathbf{p},+}^{-} ;$ the function $C_{+}(\mathbf{k}, \mathbf{k}-\mathbf{p})$, defined in (3.16) below and represented by the small circle in Fig. 4, is vanishing (like the term $\hat{\Delta}_{+}^{2,1}$ itself) if $C_{h, 0}^{-1}=1$, that is the correction term would vanish if no cutoffs (which break Gauge invariance) were present in the model.

Remark. The above Ward identity is usually stated in the physical literature by neglecting the correction term $\hat{\Delta}_{+}^{2,1}$; we shall call in general formal Ward identities the Ward identities one obtains by putting equal to 0 the correction terms. The formal Ward identities are generally derived, see [DL], [S], [MD], by neglecting the cutoffs, so that the propagator becomes simply $D_{\omega}(\mathbf{k})^{-1}$ and one can use the following relation

$$
D_{\omega}(\mathbf{p})^{-1}\left(D_{\omega}(\mathbf{k})^{-1}-D_{\omega}(\mathbf{k}+\mathbf{p})^{-1}\right)=D_{\omega}(\mathbf{k})^{-1} D_{\omega}(\mathbf{k}+\mathbf{p})^{-1} .
$$

After the derivation of the formal Ward identities, the cutoffs are introduced in order to have non diverging quantities; this approximation leads however to some well known inconsistencies, see [G].

The use of Ward identities is to provide relations between Schwinger functions, but the correction terms (due to the cutoffs) substantially affect the Ward identities and apparently spoil them of their utility. However there are other remarkable relations connecting the correction terms to the Schwinger functions; such correction identities can be proved by performing a careful analysis of the renormalized expansion for the correction terms, and come out of the peculiar properties of the function $C_{+}(\mathbf{k}, \mathbf{k}-\mathbf{p})$, see [BM2] and section 4. For example, the analysis of [BM2] implies that $\hat{\Delta}_{+}^{2,1}$ verifies the following correction identity, see Fig.5

$$
\hat{\Delta}_{+}^{2,1}(\mathbf{p}, \mathbf{k}, \mathbf{q})=\nu_{+} D_{+}(\mathbf{p}) \hat{G}_{+}^{2,1}(\mathbf{p}, \mathbf{k}, \mathbf{q})+\nu_{-} D_{-}(\mathbf{p}) \hat{G}_{-}^{2,1}(\mathbf{p}, \mathbf{k}, \mathbf{q})+\hat{H}_{+}^{2,1}(\mathbf{p}, \mathbf{k}, \mathbf{q})
$$

where $\nu_{+}, \nu_{-}$are $O(\lambda)$ and weakly dependent on $h$, once we prove that $\lambda_{j}$ is small enough for $j \geq h$, and $\hat{H}_{+}^{2,1}(\mathbf{p}, \mathbf{k}, \mathbf{q})$ can be obtained through the analogue of (1.6), from a functional integral very similar to (1.5), with the difference that $\sum_{\omega} \int d \mathbf{p} d \mathbf{k} \hat{J}_{\mathbf{p}, \omega} \psi_{\mathbf{k}, \omega}^{+} \psi_{\mathbf{k}-\mathbf{p}, \omega}^{-}$is replaced by

$$
\int d \mathbf{p} d \mathbf{k} C_{+}(\mathbf{k}, \mathbf{k}-\mathbf{p}) \hat{J}_{\mathbf{p}} \psi_{\mathbf{k},+}^{+} \psi_{\mathbf{k}-\mathbf{p},+}^{-}-\sum_{\omega} \nu_{\omega} \int d \mathbf{p} d \mathbf{k} \hat{J}_{\mathbf{p}} D_{\omega}(\mathbf{p}) \psi_{\mathbf{k}, \omega}^{+} \psi_{\mathbf{k}-\mathbf{p}, \omega}^{+}
$$

The crucial point is that $\hat{H}_{+}^{2,1}$, when computed for momenta at the cut-off scale, is $O\left(\gamma^{\theta h}\right)$ smaller, with $0<\theta<1$ a positive constant, with respect to the first two addends of the r.h.s. of (1.13). In other words the correction identity (1.13) says that the correction term $\hat{\Delta}_{+}^{2,1}$, which is usually 
neglected, can be written in terms of the Schwinger functions $\hat{G}_{+}^{2,1}$ and $\hat{G}_{-}^{2,1}$ up to the exponentially smaller term $\hat{H}_{+}^{2,1}$.

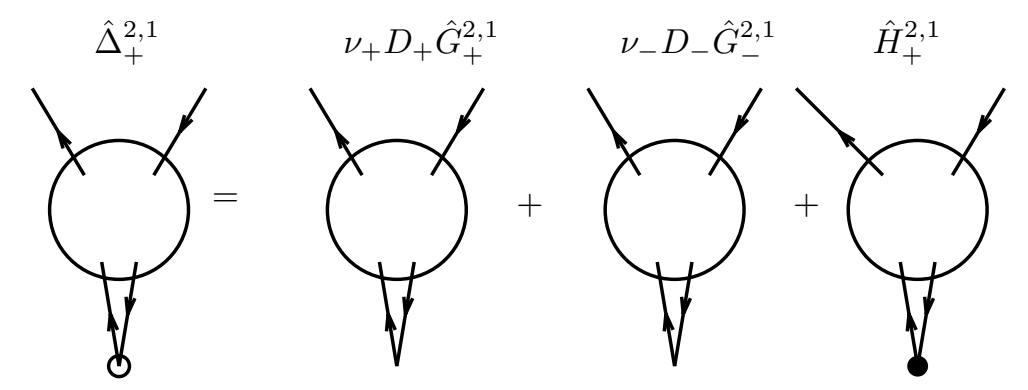

Fig. 5: Graphical representation of the correction identity (1.13); the filled point in the last term represents (1.14)

Note that (1.13) was not explicitly stated in [BM2], but its proof, which we omit here too, is implicitly contained in the proof of Theorem 4 of that paper. One has to use a strategy similar (but much simpler) to that used in $\S 4.3$ below.

Inserting the correction identity (1.13) in the Ward identity (3.14), we obtain the new identity

$$
\left(1-\nu_{+}\right) D_{+}(\mathbf{p}) \hat{G}_{+}^{2,1}(\mathbf{p}, \mathbf{k}, \mathbf{q})-\nu_{-} D_{-}(\mathbf{p}) \hat{G}_{-}^{2,1}(\mathbf{p}, \mathbf{k}, \mathbf{q})=\hat{G}_{+}^{2}(\mathbf{q})-\hat{G}_{+}^{2}(\mathbf{k})+\hat{H}_{+}^{2,1}(\mathbf{p}, \mathbf{k}, \mathbf{q})
$$

In the same way one can show that the formal Ward identity $D_{-}(\mathbf{p}) \hat{G}_{-}^{2,1}(\mathbf{p}, \mathbf{k}, \mathbf{q})=0$ becomes, if the cutoffs are taken into account

$$
\left(1-\nu_{-}^{\prime}\right) D_{-}(\mathbf{p}) \hat{G}_{-}^{2,1}(\mathbf{p}, \mathbf{k}, \mathbf{q})-\nu_{+}^{\prime} D_{+}(\mathbf{p}) \hat{G}_{+}^{2,1}(\mathbf{p}, \mathbf{k}, \mathbf{q})=\hat{H}_{-}^{2,1}(\mathbf{p}, \mathbf{k}, \mathbf{q})
$$

where, by symmetry reasons, $\nu_{ \pm}^{\prime}=\nu_{\mp}$ and $H_{-}^{2,1}$ satisfying a bound similar to that of $H_{+}^{2,1}$, when computed for momenta at the cutoff scale.

Remark. By removing both the ultraviolet and the infrared cutoff, see the Appendix, the functions $\hat{H}_{ \pm}^{2,1}$ vanishes, if we fix the momenta to some cutoff independent values. Hence the formal Ward identities are not true, even after the removal of the infrared and ultraviolet cutoff, but must be replaced by (1.15) and (1.16), with $\hat{H}_{ \pm}^{2,1}=0$. In other words, the presence of cutoffs produces modifications to the formal Ward identities, which persist when the cutoffs are removed, a phenomenon known as anomaly; in the case of Ward identities based on chiral gauge transformations, one speaks of chiral anomaly, see [Z].

The identities (1.15) and (1.16) allow us to write $\hat{G}_{+}^{2,1}$ in terms of $\hat{G}_{2}$ and $\hat{H}_{ \pm}^{2,1}$. If we put the expression so obtained in the first addend of (1.11), we can prove that it is indeed proportional to $\lambda$ with the "right" proportionality constant, see (3.30), (3.31) below. Note that in (3.31) we make reference to a bound explicitly proved in [BM2], which is expressed directly in terms of the function $\Delta_{+}^{2,1}$. In the following section we shall explain how a similar strategy can be applied to the second addend of (1.11).

1.5 Ward identities and the second addend of (1.11).

The analysis of the second addend of (1.11) is more complex, the reason being that $\mathbf{p}$ is integrated instead of being fixed at the infrared cutoff scale, as it was the case for the first addend. If we simply compute $\hat{G}_{\omega}^{4,1}$ by our series expansion and we insert it in the second addend of (1.11), we get a "bad" bound, just by dimensional reasons. We can however derive a Ward identity for $\hat{G}^{4,1}$, in the form of (3.17) below, see Fig. 6. 


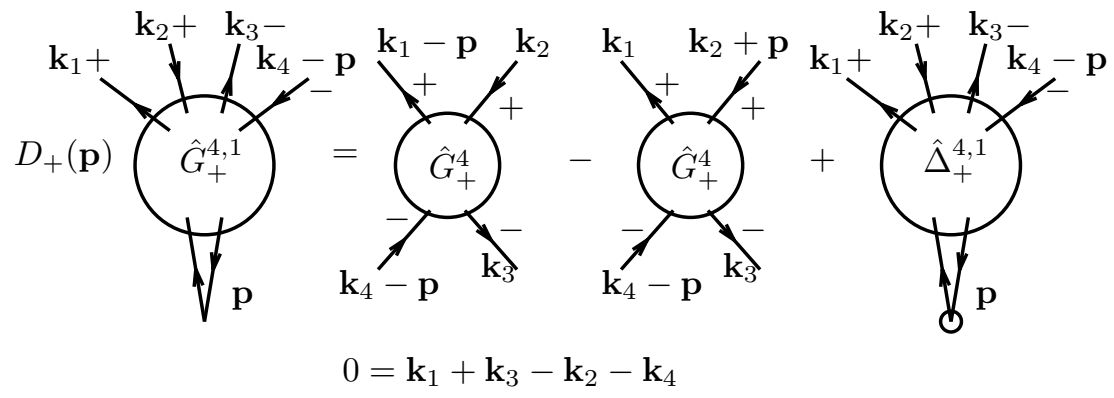

Fig. 6: Graphical representation of the Ward identity (3.17)

If we insert the above identity in the second addend of the Dyson equation, we get three terms (all multiplied by $\lambda$ ); two of them, the ones involving the $\hat{G}_{+}^{4}$ functions (which are of the form $\left.g\left(\mathbf{k}_{4}\right)(L \beta)^{-1} \sum_{\mathbf{p}} \hat{G}_{+}^{4} D_{+}(\mathbf{p})^{-1}\right)$, admit good bounds, see (3.33) below. On the contrary, the third term, which is of the form $g\left(\mathbf{k}_{4}\right)(L \beta)^{-1} \sum_{\mathbf{p}} \hat{\Delta}_{+}^{4,1} D_{+}(\mathbf{p})^{-1}$, has a "bad" bound, see [BM3]. This is not surprising, as also $\hat{\Delta}_{+}^{4,1}$ verifies a correction identity, which represents it in terms of $\hat{G}_{+}^{4,1}$ and $\hat{G}_{-}^{4,1}$; see (3.23) below and Fig. 7 .

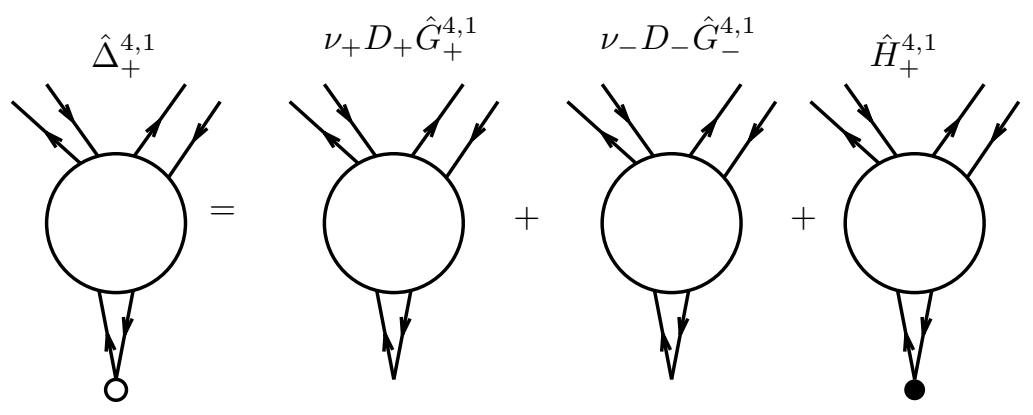

Fig. 7: The correction identity for $\hat{\Delta}_{\omega}^{4,1}$; the filled point in the last term represents (1.14)

By combining the above equation and the Ward identities for $\hat{G}_{+}^{4,1}$ and $\hat{G}_{-}^{4,1}$ we obtain, after some algebra, an equation relating $\hat{G}_{+}^{4,1}$ to $\hat{G}_{+}^{4}$ and functions $\hat{H}_{+}^{4,1}$ and $\hat{H}_{-}^{4,1}$; see (3.24) below. Inserting this expression in the second addend of the r.h.s. of the Dyson equation, we get our final expression, see (3.26) below, for the Dyson equation, containing several terms; among them the ones still requiring a further analysis are the ones involving the functions $\hat{H}_{ \pm}^{4,1}$, namely $g\left(\mathbf{k}_{4}\right)(L \beta)^{-1} \sum_{\mathbf{p}} \hat{H}_{ \pm}^{4,1} D_{+}(\mathbf{p})^{-1}$, represented as in Fig. 8. 


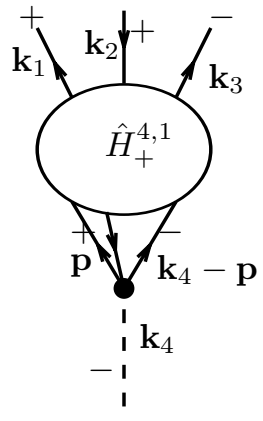

Fig. 8: Graphical representation of the term containing $\hat{H}_{ \pm}^{4,1}$

The analysis of such terms is done in $\S 4$; we again start by writing for them a Dyson equation similar to (1.11), in which the analogous of the first addend in the l.h.s. vanishes; this Dyson equation allows to write this term in terms of a function $\tilde{G}_{4}$ similar to $G_{4}$.
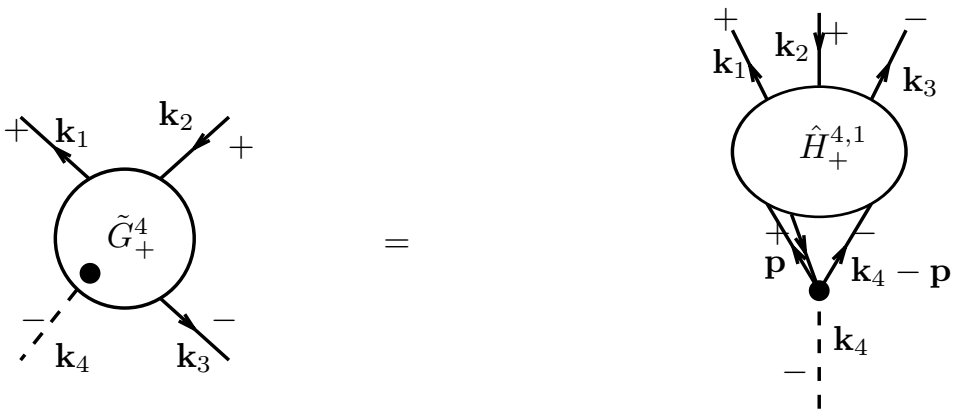

Fig. 9: Graphical representation of the Dyson equation for the correction.

We can study $\tilde{G}_{4}$ by a multiscale analysis very similar to the one for $\hat{G}^{4}$; the presence of a "special" vertex (the one associated to the filled point in Fig 9) has however the effect that a new running coupling appears, associated with the local part of the terms with four external lines among which one is the dotted line in Fig. 9, to which the bare propagator $\hat{g}\left(\mathbf{k}_{4}\right)$ is associated; we will call this new running coupling constant $\tilde{\lambda}_{j}$. It would seem that we have a problem more difficult than our initial one, since we have now to control the flow of two running coupling constants, $\lambda_{j}$ and $\tilde{\lambda}_{j}$, instead of one. However, it turns out, see Lemma 4.2 , that $\lambda_{j}$ and $\tilde{\lambda}_{j}$ are not independent but are essentially proportional; this follows from a careful analysis of the expansion for $\tilde{\lambda}_{j}$, based on the properties of the function $C_{\omega}(\mathbf{k}, \mathbf{k}-\mathbf{p})$. One then gets for $\tilde{G}_{4}$ a bound very similar to the one for $\hat{G}_{4}$, except that $\lambda_{h}$ is replaced by $\tilde{\lambda}_{h}$ (but $\tilde{\lambda}_{h}$ and $\lambda_{h}$ are proportional) and there is no wave function renormalization associated to the external line with momentum $\mathbf{k}_{4}$ (to such line is associated a "bare" instead of a "dressed" propagator, like in $\hat{G}_{4}$ ). We can however identify two class of terms in the expansion of $\tilde{G}_{4}$, see Fig. 10, and summing them has the effect that also the external line with momentum $\mathbf{k}_{4}$ is dressed by the interaction, and this allows us to complete the proof that $\lambda_{h}=\lambda+O\left(\lambda^{2}\right)$ for any $h$. 


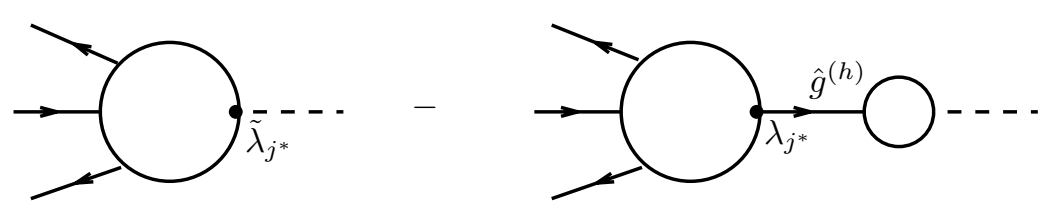

Fig. 10: The last resummation

Finally we show in the Appendix that a simple extension of our analysis implies that also the ultraviolet cutoff can be removed, that is we can construct a QFT corresponding to the Thirring model.

\section{Renormalization Group analysis}

2.1 Multiscale integration.

We resume the Renormalization Group analysis in [BM1] for the generating function (1.5). The functional integration of (1.5) can be performed iteratively in the following way. We prove by induction that, for any negative $j$, there are a constant $E_{j}$, a positive function $\tilde{Z}_{j}(\mathbf{k})$ and functionals $\mathcal{V}^{(j)}$ and $\mathcal{B}^{(j)}$ such that

$$
e^{\mathcal{W}(\phi, J)}=e^{-L \beta E_{j}} \int P_{\tilde{Z}_{j}, C_{h, j}}\left(d \psi^{[h, j]}\right) e^{-\mathcal{V}^{(j)}\left(\sqrt{Z_{j}} \psi^{[h, j]}\right)+\mathcal{B}^{(j)}\left(\sqrt{Z_{j}} \psi^{[h, j]}, \phi, J\right)},
$$

where:

1) $P_{\tilde{Z}_{j}, C_{h, j}}\left(d \psi^{[h, j]}\right)$ is the effective Grassmannian measure at scale $j$, equal to, if $Z_{j}=\max _{\mathbf{k}} \tilde{Z}_{j}(\mathbf{k})$,

$$
\begin{aligned}
& P_{\tilde{Z}_{j}, C_{h, j}}\left(d \psi^{[h, j]}\right)= \prod_{\mathbf{k}: C_{h, j}(\mathbf{k})>0} \prod_{\omega= \pm 1} \frac{d \hat{\psi}_{\mathbf{k}, \omega}^{[h, j])+} d \hat{\psi}_{\mathbf{k}, \omega}^{[h, j]-}}{\mathcal{N}_{j}(\mathbf{k})} \\
& \cdot \exp \left\{-\frac{1}{L \beta} \sum_{\mathbf{k}} C_{h, j}(\mathbf{k}) \tilde{Z}_{j}(\mathbf{k}) \sum_{\omega \pm 1} \hat{\psi}_{\omega}^{[h, j]+} D_{\omega}(\mathbf{k}) \hat{\psi}_{\mathbf{k}, \omega}^{[h, j]-}\right\} \\
& \mathcal{N}_{j}(\mathbf{k})=(L \beta)^{-1} C_{h, j}(\mathbf{k}) \tilde{Z}_{j}(\mathbf{k})\left[-k_{0}^{2}-k^{2}\right]^{1 / 2} \\
& C_{h, j}(\mathbf{k})^{-1}=\sum_{r=h}^{j} f_{r}(\mathbf{k}) \equiv \chi_{h, j}(\mathbf{k}) \quad, \quad D_{\omega}(\mathbf{k})=-i k_{0}+\omega k
\end{aligned}
$$

2) the effective potential on scale $j, \mathcal{V}^{(j)}(\psi)$, is a sum of monomial of Grassmannian variables multiplied by suitable kernels. i.e. it is of the form

$$
\mathcal{V}^{(j)}(\psi)=\sum_{n=1}^{\infty} \frac{1}{(L \beta)^{2 n}} \sum_{\substack{\mathbf{k}_{1}, \ldots, \mathbf{k}_{2 n} \\ \omega_{1}, \ldots, \omega_{2 n}}} \prod_{i=1}^{2 n} \hat{\psi}_{\mathbf{k}_{i}, \omega_{i}}^{\sigma_{i}} \hat{W}_{2 n, \underline{\omega}}^{(j)}\left(\mathbf{k}_{1}, \ldots, \mathbf{k}_{2 n-1}\right) \delta\left(\sum_{i=1}^{2 n} \sigma_{i} \mathbf{k}_{i}\right)
$$

where $\sigma_{i}=+$ for $i=1, \ldots, n, \sigma_{i}=-$ for $i=n+1, \ldots, 2 n$ and $\underline{\omega}=\left(\omega_{1}, \ldots, \omega_{2 n}\right)$;

3) the effective source term at scale $j, \mathcal{B}^{(j)}\left(\sqrt{Z_{j}} \psi, \phi, J\right)$, is a sum of monomials of Grassmannian variables and $\phi^{ \pm}, J$ field, with at least one $\phi^{ \pm}$or one $J$ field; we shall write it in the form

$$
\mathcal{B}^{(j)}\left(\sqrt{Z_{j}} \psi, \phi, J\right)=\mathcal{B}_{\phi}^{(j)}\left(\sqrt{Z_{j}} \psi\right)+\mathcal{B}_{J}^{(j)}\left(\sqrt{Z_{j}} \psi\right)+W_{R}^{(j)}\left(\sqrt{Z_{j}} \psi, \phi, J\right)
$$


where $\mathcal{B}_{\phi}^{(j)}(\psi)$ and $\mathcal{B}_{J}^{(j)}(\psi)$ denote the sums over the terms containing only one $\phi$ or $J$ field, respectively.

Of course (2.1) is true for $j=0$, with

$$
\begin{array}{ll}
\tilde{Z}_{0}(\mathbf{k})=1, \quad E_{0}=0, \quad \mathcal{V}^{(0)}(\psi)=V(\psi), & W_{R}^{(0)}=0 \\
\mathcal{B}_{\phi}^{(0)}(\psi)=\sum_{\omega} \int d \mathbf{x}\left[\phi_{\mathbf{x}, \omega}^{+} \psi_{\mathbf{x}, \omega}^{-}+\psi_{\mathbf{x}, \omega}^{+} \phi_{\mathbf{x}, \omega}^{-}\right], & \mathcal{B}_{J}^{(0)}(\psi)=\sum_{\omega} \int d \mathbf{x} J_{\mathbf{x}, \omega} \psi_{\mathbf{x}, \omega}^{+} \psi_{\mathbf{x}, \omega}^{-} .
\end{array}
$$

Let us now assume that (2.1) is satisfied for a certain $j \leq 0$ and let us show that it holds also with $j-1$ in place of $j$.

In order to perform the integration corresponding to $\psi^{(j)}$, we write the effective potential and the effective source as sum of two terms, according to the following rules.

We split the effective potential $\mathcal{V}^{(j)}$ as $\mathcal{L} \mathcal{V}^{(j)}+\mathcal{R} \mathcal{V}^{(j)}$, where $\mathcal{R}=1-\mathcal{L}$ and $\mathcal{L}$, the localization operator, is a linear operator on functions of the form (2.5), defined in the following way by its action on the kernels $\hat{W}_{2 n, \underline{\omega}}^{(j)}$.

1) If $2 n=4$, then

$$
\mathcal{L} \hat{W}_{4, \underline{\omega}}^{(j)}\left(\mathbf{k}_{1}, \mathbf{k}_{2}, \mathbf{k}_{3}\right)=\hat{W}_{4, \underline{\omega}}^{(j)}\left(\overline{\mathbf{k}}_{++}, \overline{\mathbf{k}}_{++}, \overline{\mathbf{k}}_{++}\right),
$$

where $\overline{\mathbf{k}}_{\eta \eta^{\prime}}=\left(\eta \pi L^{-1}, \eta^{\prime} \pi \beta^{-1}\right)$. Note that $\mathcal{L} \hat{W}_{4, \underline{\omega}}^{(j)}\left(\mathbf{k}_{1}, \mathbf{k}_{2}, \mathbf{k}_{3}\right)=0$, if $\sum_{i=1}^{4} \omega_{i} \neq 0$, by simple symmetry considerations.

2) If $2 n=2$ (in this case there is a non zero contribution only if $\omega_{1}=\omega_{2}$ )

$$
\mathcal{L} \hat{W}_{2, \underline{\omega}}^{(j)}(\mathbf{k})=\frac{1}{4} \sum_{\eta, \eta^{\prime}= \pm 1} \hat{W}_{2, \underline{\omega}}^{(j)}\left(\overline{\mathbf{k}}_{\eta \eta^{\prime}}\right)\left\{1+\eta \frac{L}{\pi}+\eta^{\prime} \frac{\beta}{\pi} k_{0}\right\}
$$

3) In all the other cases

$$
\mathcal{L} \hat{W}_{2 n, \underline{\omega}}^{(j)}\left(\mathbf{k}_{1}, \ldots, \mathbf{k}_{2 n-1}\right)=0
$$

These definitions are such that $\mathcal{L}^{2}=\mathcal{L}$, a property which plays an important role in the analysis of [BM1]. Moreover, by using the symmetries of the model, it is easy to see that

$$
\mathcal{L V}^{(j)}\left(\psi^{[h, j]}\right)=z_{j} F_{\zeta}^{[h, j]}+a_{j} F_{\alpha}^{[h, j]}+l_{j} F_{\lambda}^{[h, j]},
$$

where $z_{j}, a_{j}$ and $l_{j}$ are real numbers and

$$
\begin{aligned}
& F_{\alpha}^{[h, j]}=\sum_{\omega} \frac{\omega}{(L \beta)} \sum_{\mathbf{k}: C_{h, j}^{-1}(\mathbf{k})>0} k \hat{\psi}_{\mathbf{k}, \omega}^{[h, j]+} \hat{\psi}_{\mathbf{k}, \omega}^{[h, j]-}=\sum_{\omega} i \omega \int_{\Lambda} d \mathbf{x} \psi_{\mathbf{x}, \omega}^{[h, j]+} \partial_{x} \psi_{\mathbf{x}, \omega}^{[h, j]-}, \\
& F_{\zeta}^{[h, j]}=\sum_{\omega} \frac{1}{(L \beta)} \sum_{\mathbf{k}: C_{h, j}^{-1}(\mathbf{k})>0}\left(-i k_{0}\right) \hat{\psi}_{\mathbf{k}, \omega}^{[h, j]+} \hat{\psi}_{\mathbf{k}^{\prime}, \omega}^{[h, j]-}=-\sum_{\omega} \int_{\Lambda} d \mathbf{x} \psi_{\mathbf{x}, \omega}^{[h, j]+} \partial_{0} \psi_{\mathbf{x}, \omega}^{[h, j]-}, \\
& F_{\lambda}^{[h, j]}=\frac{1}{(L \beta)^{4}} \sum_{\mathbf{k}_{1}, \ldots, \mathbf{k}_{4}: C_{h, j}^{-1}\left(\mathbf{k}_{i}\right)>0} \hat{\psi}_{\mathbf{k}_{1},+}^{[h, j]+} \hat{\psi}_{\mathbf{k}_{2},+}^{[h, j]-} \hat{\psi}_{\mathbf{k}_{3},-}^{[h, j]+} \hat{\psi}_{\mathbf{k}_{4},-}^{[h, j]-} \delta\left(\mathbf{k}_{1}-\mathbf{k}_{2}+\mathbf{k}_{3}-\mathbf{k}_{4}\right)
\end{aligned}
$$

$\partial_{x}$ and $\partial_{0}$ are defined in an obvious way, so that the second equality in (2.12) and (2.13) is satisfied; if $N=\infty$ they are simply the partial derivative with respect to $x$ and $x_{0}$. Note that $\mathcal{L} \mathcal{V}^{(0)}=\mathcal{V}^{(0)}$, hence $l_{0}=\lambda, a_{0}=z_{0}=0$.

In the limit $L=\beta=\infty$ one has $a_{j}=z_{j}$ as a trivial consequence of the symmetries of the propagator. If $L, \beta$ are finite this is not true and by dimensional arguments it follows that $z_{j}-a_{j}$ is of order $\gamma^{-j} \max \left\{L^{-1}, \beta^{-1}\right\}$. 
Analogously we write $\mathcal{B}^{(j)}=\mathcal{L B}^{(j)}+\mathcal{R} \mathcal{B}^{(j)}, \mathcal{R}=1-\mathcal{L}$, according to the following definition. First of all, we put $\mathcal{L} W_{R}^{(j)}=W_{R}^{(j)}$. Let us consider now $\mathcal{B}_{J}^{(j)}\left(\sqrt{Z_{j}} \psi\right)$. It is easy to see that the field $J$ is equivalent, from the point of view of dimensional considerations, to two $\psi$ fields. Hence, the only terms which need to be renormalized are those of second order in $\psi$, which are indeed marginal. We shall use for them the definition

$$
\begin{aligned}
\mathcal{B}_{J}^{(j, 2)}\left(\sqrt{Z_{j}} \psi\right) & =\sum_{\omega, \tilde{\omega}} \int d \mathbf{x} d \mathbf{y} d \mathbf{z} B_{\omega, \tilde{\omega}}(\mathbf{x}, \mathbf{y}, \mathbf{z}) J_{\mathbf{x}, \omega}\left(\sqrt{Z_{j}} \psi_{\mathbf{y}, \tilde{\omega}}^{+}\right)\left(\sqrt{Z_{j}} \psi_{\mathbf{z}, \tilde{\omega}}^{-}\right)= \\
& =\sum_{\omega, \tilde{\omega}} \int \frac{d \mathbf{p}}{(2 \pi)^{2}} \frac{d \mathbf{k}}{(2 \pi)^{2}} \hat{B}_{\omega, \tilde{\omega}}(\mathbf{p}, \mathbf{k}) \hat{J}(\mathbf{p})\left(\sqrt{Z_{j}} \hat{\psi}_{\mathbf{p}+\mathbf{k}, \tilde{\omega}}^{+}\right)\left(\sqrt{Z_{j}} \hat{\psi}_{\mathbf{k}, \tilde{\omega}}^{-}\right) .
\end{aligned}
$$

We regularize $\mathcal{B}_{J}^{(j, 2)}\left(\sqrt{Z_{j}} \psi\right)$, in analogy to what we did for the effective potential, by decomposing it as the sum of $\mathcal{L B}_{J}^{(j, 2)}\left(\sqrt{Z_{j}} \psi\right)$ and $\mathcal{R B}_{J}^{(j, 2)}\left(\sqrt{Z_{j}} \psi\right)$, where $\mathcal{L}$ is defined through its action on $\hat{B}_{\omega}(\mathbf{p}, \mathbf{k})$ in the following way:

$$
\mathcal{L} \hat{B}_{\omega, \tilde{\omega}}(\mathbf{p}, \mathbf{k})=\frac{1}{4} \sum_{\eta, \eta^{\prime}= \pm 1} \hat{B}_{\omega, \tilde{\omega}}\left(\bar{p}_{\eta}, \bar{k}_{\eta, \eta^{\prime}}\right)
$$

where $\bar{k}_{\eta, \eta^{\prime}}$ was defined above and $\bar{p}_{\eta}=\left(0,2 \pi \eta^{\prime} / \beta\right)$. In the limit $L=\beta=\infty$ it reduces simply to $\mathcal{L} \hat{B}_{\omega, \tilde{\omega}}(\mathbf{p}, \mathbf{k})=\hat{B}_{\omega, \tilde{\omega}}(0,0)$.

This definition apparently implies that we have to introduce two new renormalization constants. However, one can easily show that, in the limit $L, \beta \rightarrow \infty, \hat{B}_{\omega,-\omega}(0,0)=0$, while, at finite $L$ and $\beta, \mathcal{L} B_{\omega,-\omega}$ behaves as an irrelevant term, see [BM1].

The previous considerations imply that we can write

$$
\mathcal{L B}_{J}^{(j, 2)}\left(\sqrt{Z_{j}} \psi\right)=\sum_{\omega} \frac{Z_{j}^{(2)}}{Z_{j}} \int d \mathbf{x} J_{\mathbf{x}, \omega}\left(\sqrt{Z_{j}} \psi_{\mathbf{x}, \omega}^{+}\right)\left(\sqrt{Z_{j}} \psi_{\mathbf{x}, \omega}^{-}\right)
$$

which defines the renormalization constant $Z_{j}^{(2)}$.

Finally we have to define $\mathcal{L}$ for $\mathcal{B}_{\phi}^{(j)}\left(\sqrt{Z_{j}} \psi\right)$; we want to show that, by a suitable choice of the localization procedure, if $j \leq-1$, it can be written in the form

$$
\begin{gathered}
\mathcal{B}_{\phi}^{(j)}\left(\sqrt{Z_{j}} \psi\right)=\sum_{\omega} \sum_{i=j+1}^{0} \int d \mathbf{x} d \mathbf{y} \cdot \\
\cdot\left[\phi_{\mathbf{x}, \omega}^{+} g_{\omega}^{Q,(i)}(\mathbf{x}-\mathbf{y}) \frac{\partial}{\partial \psi_{\mathbf{y} \omega}^{+}} \mathcal{V}^{(j)}\left(\sqrt{Z_{j}} \psi\right)+\frac{\partial}{\partial \psi_{\mathbf{y}, \omega}^{-}} \mathcal{V}^{(j)}\left(\sqrt{Z_{j}} \psi\right) g_{\omega}^{Q,(i)}(\mathbf{y}-\mathbf{x}) \phi_{\mathbf{x}, \omega}^{-}\right]+ \\
+\sum_{\omega} \int \frac{d \mathbf{k}}{(2 \pi)^{2}}\left[\hat{\psi}_{\mathbf{k}, \omega}^{h, j]+} \hat{Q}_{\omega}^{(j+1)}(\mathbf{k}) \hat{\phi}_{\mathbf{k}, \omega}^{-}+\hat{\phi}_{\mathbf{k}, \omega}^{+} \hat{Q}_{\omega}^{(j+1)}(\mathbf{k}) \hat{\psi}_{\mathbf{k}, \omega}^{[h, j]-}\right]
\end{gathered}
$$

where $\hat{g}_{\omega}^{Q,(i)}(\mathbf{k})=\hat{g}_{\omega}^{(i)}(\mathbf{k}) \hat{Q}_{\omega}^{(i)}(\mathbf{k})$, with

$$
\hat{g}_{\omega}^{(j)}(\mathbf{k})=\frac{1}{Z_{j-1}} \frac{\tilde{f}_{j}(\mathbf{k})}{D_{\omega}(\mathbf{k})}
$$

$\tilde{f}_{j}(\mathbf{k})=f_{j}(\mathbf{k}) Z_{j-1}\left[\tilde{Z}_{j-1}(\mathbf{k})\right]^{-1}$ and $Q_{\omega}^{(j)}(\mathbf{k})$ defined inductively by the relations

$$
\hat{Q}_{\omega}^{(j)}(\mathbf{k})=\hat{Q}_{\omega}^{(j+1)}(\mathbf{k})-z_{j} Z_{j} D_{\omega}(\mathbf{k}) \sum_{i=j+1}^{0} \hat{g}_{\omega}^{Q,(i)}(\mathbf{k}), \quad \hat{Q}_{\omega}^{(0)}(\mathbf{k})=1 .
$$


Note that $\hat{g}_{\omega}^{(j)}(\mathbf{k})$ does not depend on the infrared cutoff for $j>h$ and that (even for $\left.j=h\right) \hat{g}^{(j)}(\mathbf{k})$ is of size $Z_{j-1}^{-1} \gamma^{-j}$, see discussion in $§ 3$ of [BM2], after eq. (60). Moreover the propagator $\hat{g}_{\omega}^{Q,(i)}(\mathbf{k})$ is equivalent to $\hat{g}_{\omega}^{(i)}(\mathbf{k})$, as concerns the dimensional bounds.

The $\mathcal{L}$ operation for $\mathcal{B}_{\phi}^{(j)}$ is defined by decomposing $\mathcal{V}^{(j)}$ in the r.h.s. of $(2.18)$ as $\mathcal{L} \mathcal{V}^{(j)}+\mathcal{R} \mathcal{V}^{(j)}$, $\mathcal{L} \mathcal{V}^{(j)}$ being defined by $(2.11)$.

After writing $\mathcal{V}^{(j)}=\mathcal{L} \mathcal{V}^{(j)}+\mathcal{R} \mathcal{V}^{(j)}$ and $\mathcal{B}^{(j)}=\mathcal{L} \mathcal{B}^{(j)}+\mathcal{R} \mathcal{B}^{(j)}$, the next step is to renormalize the free measure $P_{\tilde{Z}_{j}, C_{h, j}}\left(d \psi^{[h, j]}\right)$, by adding to it part of the r.h.s. of (2.11). We get

$$
\begin{aligned}
& \int P_{\tilde{Z}_{j}, C_{h, j}}\left(d \psi^{[h, j]}\right) e^{-\mathcal{V}^{(j)}\left(\sqrt{Z_{j}} \psi^{[h, j]}\right)+\mathcal{B}^{(j)}\left(\sqrt{Z_{j}} \psi^{[h, j]}\right)}= \\
& =e^{-L \beta t_{j}} \int P_{\tilde{Z}_{j-1}, C_{h, j}}\left(d \psi^{[h, j]}\right) e^{-\tilde{\mathcal{V}}^{(j)}\left(\sqrt{Z_{j}} \psi^{[h, j]}\right)+\tilde{\mathcal{B}}^{(j)}\left(\sqrt{Z_{j}} \psi^{[h, j]}\right),}
\end{aligned}
$$

where

$$
\begin{gathered}
\tilde{Z}_{j-1}(\mathbf{k})=Z_{j}(\mathbf{k})\left[1+\chi_{h, j}(\mathbf{k}) z_{j}\right], \\
\tilde{\mathcal{V}}^{(j)}\left(\sqrt{Z_{j}} \psi^{[h, j]}\right)=\mathcal{V}^{(j)}\left(\sqrt{Z_{j}} \psi^{[h, j]}\right)-z_{j} Z_{j}\left[F_{\zeta}^{[h, j]}+F_{\alpha}^{[h, j]}\right],
\end{gathered}
$$

and the factor $\exp \left(-L \beta t_{j}\right)$ in $(2.21)$ takes into account the different normalization of the two measures. Moreover

$$
\tilde{\mathcal{B}}^{(j)}\left(\sqrt{Z_{j}} \psi^{[h, j]}\right)=\tilde{\mathcal{B}}_{\phi}^{(j)}\left(\sqrt{Z_{j}} \psi^{[h, j]}\right)+\mathcal{B}_{J}^{(j)}\left(\sqrt{Z_{j}} \psi^{[h, j]}\right)+W_{R}^{(j)},
$$

where $\tilde{\mathcal{B}}_{\phi}^{(j)}$ is obtained from $\mathcal{B}_{\phi}^{(j)}$ by inserting (2.23) in the second line of (2.18) and by absorbing the terms proportional to $z_{j}$ in the terms in the third line of (2.18).

If $j>h$, the r.h.s of $(2.21)$ can be written as

$$
\begin{gathered}
e^{-L \beta t_{j}} \int P_{\tilde{Z}_{j-1}, C_{h, j-1}}\left(d \psi^{[h, j-1]}\right) \int P_{Z_{j-1}, \tilde{f}_{j}^{-1}}\left(d \psi^{(j)}\right) \\
e^{-\tilde{\mathcal{V}}^{(j)}\left(\sqrt{Z_{j}}\left[\psi^{[h, j-1]}+\psi^{(j)}\right]\right)+\tilde{\mathcal{B}}^{(j)}}\left(\sqrt{Z_{j}}\left[\psi^{[h, j-1]}+\psi^{(j)}\right]\right),
\end{gathered}
$$

where $P_{Z_{j-1}, \tilde{f}_{j}^{-1}}\left(d \psi^{(j)}\right)$ is the integration with propagator $\hat{g}_{\omega}^{(j)}(\mathbf{k})$.

We now rescale the field so that

$$
\tilde{\mathcal{V}}^{(j)}\left(\sqrt{Z_{j}} \psi^{[h, j]}\right)=\hat{\mathcal{V}}^{(j)}\left(\sqrt{Z_{j-1}} \psi^{[h, j]}\right) \quad, \quad \tilde{\mathcal{B}}^{(j)}\left(\sqrt{Z_{j}} \psi^{[h, j]}\right)=\hat{\mathcal{B}}^{(j)}\left(\sqrt{Z_{j-1}} \psi^{[h, j]}\right)
$$

it follows that (in the limit $L, \beta=\infty$, so that $a_{j}=z_{j}$, see above)

$$
\mathcal{L} \hat{\mathcal{V}}^{(j)}\left(\psi^{[h, j]}\right)=\lambda_{j} F_{\lambda}^{[h, j]},
$$

where $\lambda_{j}=\left(Z_{j} Z_{j-1}^{-1}\right)^{2} l_{j}$. If we now define

$$
\begin{aligned}
& e^{-\mathcal{V}^{(j-1)}\left(\sqrt{Z_{j-1}} \psi^{[h, j-1]}\right)+\mathcal{B}^{(j-1)}\left(\sqrt{Z_{j-1}} \psi^{[h, j-1]}\right)-L \beta E_{j}}= \\
& =\int P_{Z_{j-1}, \tilde{f}_{j}^{-1}}\left(d \psi^{(j)}\right) e^{-\hat{\mathcal{V}}^{(j)}\left(\sqrt{Z_{j-1}}\left[\psi^{[h, j-1]}+\psi^{(j)}\right]\right)+\hat{\mathcal{B}}^{(j)}\left(\sqrt{Z_{j-1}}\left[\psi^{[h, j-1]}+\psi^{(j)}\right]\right),}
\end{aligned}
$$

it is easy to see that $\mathcal{V}^{(j-1)}$ and $\mathcal{B}^{(j-1)}$ are of the same form of $\mathcal{V}^{(j)}$ and $\mathcal{B}^{(j)}$ and that the procedure can be iterated. Note that the above procedure allows, in particular, to write the running coupling constant $\lambda_{j}, 0<j \leq h$, in terms of $\lambda_{j^{\prime}}, 0 \geq j^{\prime} \geq j+1$ :

$$
\lambda_{j}=\beta_{j}^{(h)}\left(\lambda_{j+1}, \ldots, \lambda_{0}\right) \quad, \quad \lambda_{0}=\lambda,
$$


The function $\beta_{j}^{(h)}\left(\lambda_{j+1}, \ldots, \lambda_{0}\right)$ is called the Beta function. By the remark above on the independence of scale $j$ propagators of $h$ for $j>h$, it is independent of $h$, for $j>h$.

2.2 Tree expansion.

At the end of the iterative integration procedure, we get

$$
\mathcal{W}(\varphi, J)=-L \beta E_{L, \beta}+\sum_{m^{\phi}+n^{J} \geq 1} S_{2 m^{\phi}, n^{J}}^{(h)}(\phi, J)
$$

where $E_{L, \beta}$ is the free energy and $S_{2 m^{\phi}, n^{J}}^{(h)}(\phi, J)$ are suitable functional, which can be expanded, as well as $E_{L, \beta}$, the effective potentials and the various terms in the r.h.s. of (2.6) and (2.5), in terms of trees (for an updated introduction to trees formalism see also [GM]). This expansion, which is indeed a finite sum for finite values of $N, L, \beta$, is explained in detail in [BGPS] and [BM1], which we shall refer to often in the following.

Let us consider the family of all trees which can be constructed by joining a point $r$, the root, with an ordered set of $n \geq 1$ points, the endpoints of the unlabelled tree, so that $r$ is not a branching point. Two unlabelled trees are identified if they can be superposed by a suitable continuous deformation, so that the endpoints with the same index coincide.

$n$ will be called the order of the unlabelled tree and the branching points will be called the non trivial vertices. The unlabelled trees are partially ordered from the root to the endpoints in the natural way; we shall use the symbol $<$ to denote the partial order.

We shall consider also the labelled trees (to be called simply trees in the following), see Fig. 11; they are defined by associating some labels with the unlabelled trees, as explained in the following items.

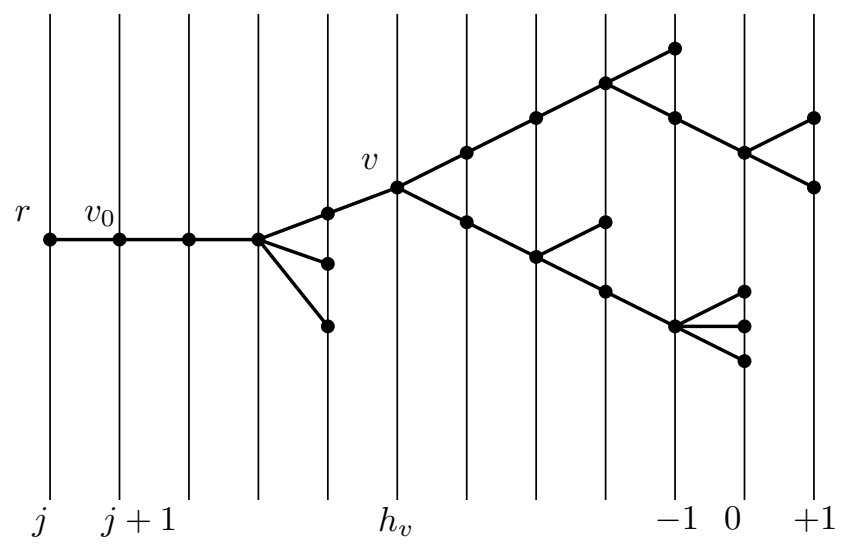

Fig. 11: A tree $\tau$ and its labels.

1) We associate a label $j \leq 0$ with the root and we denote $\mathcal{T}_{j, n}$ the corresponding set of labelled trees with $n$ endpoints. Moreover, we introduce a family of vertical lines, labelled by an integer taking values in $[j, 1]$, and we represent any tree $\tau \in \mathcal{T}_{j, n}$ so that, if $v$ is an endpoint or a non trivial vertex, it is contained in a vertical line with index $h_{v}>j$, to be called the scale of $v$, while the root is on the line with index $j$. There is the constraint that, if $v$ is an endpoint, $h_{v}>j+1$.

The tree will intersect in general the vertical lines in set of points different from the root, the endpoints and the non trivial vertices; these points will be called trivial vertices. The set of the vertices of $\tau$ will be the union of the endpoints, the trivial vertices and the non trivial vertices. The definition of $h_{v}$ is extended in an obvious way to the trivial vertices and the endpoints.

Note that, if $v_{1}$ and $v_{2}$ are two vertices and $v_{1}<v_{2}$, then $h_{v_{1}}<h_{v_{2}}$. Moreover, there is only one vertex immediately following the root, which will be denoted $v_{0}$ and can not be an endpoint; its scale is $j+1$.

2) There are two kind of endpoints, normal and special.

$11 /$ ottobre/2018; $1: 16$ 
With each normal endpoint $v$ of scale $h_{v}$ we associate the local term $\mathcal{L} \hat{\mathcal{V}}^{\left(h_{v}\right)}\left(\psi^{\left[h, h_{v}-1\right]}\right)$ of $(2.27)$ and one space-time point $\mathbf{x}_{v}$. We shall say that the endpoint is of type $\lambda$.

There are two types of special endpoints, to be called of type $\phi$ and $J$; the first one is associated with the terms in the third line of (2.18), the second one with the terms in the r.h.s. of (2.17). Given $v \in \tau$, we shall call $n_{v}^{\phi}$ and $n_{v}^{J}$ the number of endpoints of type $\phi$ and $J$ following $v$ in the tree, while $n_{v}$ will denote the number of normal endpoints following $v$. Analogously, given $\tau$, we shall call $n_{\tau}^{\phi}$ and $n_{\tau}^{J}$ the number of endpoint of type $\phi$ and $J$, while $n_{\tau}$ will denote the number of normal endpoints. Finally, $\mathcal{T}_{j, n, n^{\phi}, n^{J}}$ will denote the set of trees belonging to $\mathcal{T}_{j, n}$ with $n$ normal endpoints, $n^{\phi}$ endpoints of type $\phi$ and $n^{J}$ endpoints of type $J$. Given a vertex $v$, which is not an endpoint, $\mathbf{x}_{v}$ will denote the family of all space-time points associated with one of the endpoints following $v$.

3) There is an important constraint on the scale indices of the endpoints. In fact, if $v$ is an endpoint normal or of type $J, h_{v}=h_{v^{\prime}}+1$, if $v^{\prime}$ is the non trivial vertex immediately preceding $v$. This constraint takes into account the fact that at least one of the $\psi$ fields associated with an endpoint normal or of type $J$ has to be contracted in a propagator of scale $h_{v^{\prime}}$, as a consequence of our definitions.

On the contrary, if $v$ is an endpoint of type $\phi$, we shall only impose the condition that $h_{v} \geq h_{v^{\prime}}+1$. In this case the only $\psi$ field associated with $v$ is contracted in a propagator of scale $h_{v}-1$, instead of $h_{v^{\prime}}$.

4) If $v$ is not an endpoint, the cluster $L_{v}$ with frequency $h_{v}$ is the set of endpoints following the vertex $v$; if $v$ is an endpoint, it is itself a (trivial) cluster. The tree provides an organization of endpoints into a hierarchy of clusters.

5) We associate with any vertex $v$ of the tree a set $P_{v}$, the external fields of $v$. The set $P_{v}$ includes both the field variables of type $\psi$ which belong to one of the endpoints following $v$ and are not yet contracted at scale $h_{v}$ (in the iterative integration procedure), to be called normal external fields, and those which belong to an endpoint normal or of type $J$ and are contracted with a field variable belonging to an endpoint $\tilde{v}$ of type $\phi$ through a propagator $g^{Q,\left(h_{\tilde{v}}-1\right)}$, to be called special external fields of $v$.

These subsets must satisfy various constraints. First of all, if $v$ is not an endpoint and $v_{1}, \ldots, v_{s_{v}}$ are the $s_{v}$ vertices immediately following it, then $P_{v} \subset \cup_{i} P_{v_{i}}$. We shall denote $Q_{v_{i}}$ the intersection of $P_{v}$ and $P_{v_{i}}$; this definition implies that $P_{v}=\cup_{i} Q_{v_{i}}$. The subsets $P_{v_{i}} \backslash Q_{v_{i}}$, whose union will be made, by definition, of the internal fields of $v$, have to be non empty, if $s_{v}>1$, that is if $v$ is a non trivial vertex.

Moreover, if the set $P_{v_{0}}$ contains only special external fields, that is if $\left|P_{v_{0}}\right|=n^{\phi}$, and $\tilde{v}_{0}$ is the vertex immediately following $v_{0}$, then $\left|P_{v_{0}}\right|<\left|P_{\tilde{v}_{0}}\right|$.

\subsection{Dimensional bounds.}

We can write

$$
\begin{gathered}
S_{2 m^{\phi}, n^{J}}^{(h)}(\phi, J)= \\
=\sum_{n=0}^{\infty} \sum_{j_{0}=h-1}^{-1} \sum_{\substack{\tau \in \mathcal{T} \\
j_{0}, n, 2 m^{\phi}, n^{J} \\
\left|P_{v_{0}}\right|=2 m \phi}} \sum_{\underline{\omega}} \int d \underline{\mathbf{x}} \prod_{i=1}^{2 m^{\phi}} \phi_{\mathbf{x}_{i}, \omega_{i}}^{\sigma_{i}} \prod_{r=1}^{n^{J}} J_{\mathbf{x}_{2 m^{\phi}+r}, \omega_{2 m} \phi+r} S_{2 m^{\phi}, n^{J}, \tau, \underline{\omega}}(\underline{\mathbf{x}}),
\end{gathered}
$$

where $\underline{\omega}=\underline{\omega}=\left\{\omega_{1}, \ldots, \omega_{2 m^{\phi}+n^{J}}\right\}, \underline{\mathbf{x}}=\left\{\mathbf{x}_{1}, \ldots, \mathbf{x}_{2 m^{\phi}+n^{J}}\right\}$ and $\sigma_{i}=+$ if $i$ is odd, $\sigma_{i}=-$ if $i$ is even.

Let us define $\bar{\lambda}_{j}=\max _{k \geq j}\left|\lambda_{k}\right|$; in $\S 3$ of [BM1] it is proved that the kernels satisfy the following 
bound:

$$
\begin{gathered}
\int d \underline{\mathbf{x}}\left|S_{2 m^{\phi}, n^{J}, \tau, \underline{\omega}}(\underline{\mathbf{x}})\right| \leq L \beta\left(C \bar{\lambda}_{j_{0}}\right)^{n} \gamma^{-j_{0}\left(-2+m^{\phi}+n^{J}\right)} \prod_{i=1}^{2 m^{\phi}} \frac{\gamma^{-h_{i}}}{\left(Z_{h_{i}}\right)^{1 / 2}} . \\
\cdot \prod_{r=1}^{n^{J}} \frac{Z_{\bar{h}_{r}}^{(2)}}{Z_{\bar{h}_{r}}} \prod_{\text {v not e.p }}\left(\frac{Z_{h_{v}}}{Z_{h_{v}-1}}\right)^{\left|P_{v}\right| / 2} \gamma^{-d_{v}}
\end{gathered}
$$

where $h_{i}$ is the scale of the propagator linking the $i$-th endpoint of type $\phi$ to the tree, $\bar{h}_{r}$ is the scale of the $r$-th endpoint of type $J$ and

$$
d_{v}=-2+\left|P_{v}\right| / 2+n_{v}^{J}+\tilde{z}\left(P_{v}\right)
$$

with

$$
\tilde{z}\left(P_{v}\right)= \begin{cases}z\left(P_{v}\right) & \text { if } n_{v}^{\phi} \leq 1, n_{v}^{J}=0, \\ 1 & \text { if } n_{v}^{\phi}=0, n_{v}^{J}=1,\left|P_{v}\right|=2, \\ 0 & \text { otherwise }\end{cases}
$$

and $z\left(P_{v}\right)=1$ if $\left|P_{v}\right|=4, z\left(P_{v}\right)=2$ if $\left|P_{v}\right|=2$ and zero otherwise.

As explained in $\S 5$ of [BM1], one can sum over the trees $\tau$ only if $d_{v}>0$. While it is not true in general that $d_{v}>0$ in $(2.32)$, it is true for the trees contributing to $\hat{G}_{\omega}^{2,1}, \hat{G}_{\omega}^{2}, \hat{G}_{+}^{4}$ with external momenta computed at the cutoff scale; hence by using the bound (2.32), one can prove, see [BM2] $\S 3.5$, the following theorem.

Theorem 2.1 There exists $\varepsilon_{0}$ such that, if $\bar{\lambda}_{h} \leq \varepsilon_{0}$ and $|\overline{\mathbf{k}}|=\gamma^{h}$, then

$$
\begin{gathered}
\hat{G}_{\omega}^{2,1}(2 \overline{\mathbf{k}}, \overline{\mathbf{k}})=-\frac{Z_{h}^{(2)}}{Z_{h}^{2} D_{\omega}(\overline{\mathbf{k}})^{2}}\left[1+O\left(\bar{\lambda}_{h}^{2}\right)\right], \\
\hat{G}_{\omega}^{2}(\overline{\mathbf{k}})=\frac{1}{Z_{h} D_{\omega}(\overline{\mathbf{k}})}\left[1+O\left(\bar{\lambda}_{h}^{2}\right)\right], \\
\hat{G}_{+}^{4}(\overline{\mathbf{k}},-\overline{\mathbf{k}},-\overline{\mathbf{k}})=Z_{h}^{-2}|\overline{\mathbf{k}}|^{-4}\left[-\lambda_{h}+O\left(\bar{\lambda}_{h}^{2}\right)\right] .
\end{gathered}
$$

The expansion for (2.35), (2.36), (2.37) in terms of the running coupling constant $\lambda_{j}$ is convergent if $\lambda_{j}$ is small enough for all $j \geq h$. This property is surely true if $|h|$ is at most of order $|\lambda|^{-1}$, but to prove that it is true for any $|h|$ is quite nontrivial, as it is consequence of intricate cancellations which are present in the Beta function. In the following section we will show, by using Ward identities and a Dyson equation, that indeed $\lambda_{j}$ is small enough for all $j \geq h$ for any $h$, that is uniformly in the infrared cutoff, so that the above theorem can be applied.

\section{Vanishing of Beta function}

\subsection{The main theorem}

The main result of this paper is the following theorem.

Theorem 3.1 The model (1.5) is well defined in the limit $h \rightarrow-\infty$. In fact there are constants $\varepsilon_{1}$ and $c_{2}$ such that $|\lambda| \leq \varepsilon_{1}$ implies $\bar{\lambda}_{j} \leq c_{2} \varepsilon_{1}$, for any $j<0$.

Proof - The proof of Theorem 3.1 is done by contradiction. Assume that there exists a $h \leq 0$ such that

$$
\bar{\lambda}_{h+1} \leq c_{2} \varepsilon_{1}<\left|\lambda_{h}\right| \leq 2 c_{2} \varepsilon_{1} \leq \varepsilon_{0}
$$


where $\varepsilon_{0}$ is the same as in Theorem 2.1. We show that this is not possible, if $\varepsilon_{1}, c_{2}$ are suitably chosen.

Let us consider the model with cutoff $\gamma^{h}$. In the following sections we shall prove that, in this model,

$$
\left|\lambda_{h}-\lambda\right| \leq c_{3} \bar{\lambda}_{h+1}^{2}
$$

However, as a consequence of the remark after (2.19), $\lambda_{j}$ is the same, for any $j \geq h$, in the model with or without cutoff; in fact $g^{(j)}(\mathbf{k})$ does not depend on $h$ for $j>h$, and $\lambda_{h}$ only depends on the propagators $g^{(j)}(\mathbf{k})$ with $j>h$ by definition. Hence, from (3.2) we get the bound $\left|\lambda_{h}\right| \leq \varepsilon_{1}+c_{3} c_{2}^{2} \varepsilon_{1}^{2}$, which is in contradiction with (3.1) if, for instance, $c_{2}=2$ and $\varepsilon_{1} \leq 1 /\left(4 c_{3}\right)$.

Theorem 3.1 implies, as proved in [BM3], that

$$
\left|\beta_{j, \lambda}\left(\lambda_{h}, . ., \lambda_{h}\right)\right| \leq C\left|\lambda_{h}\right|^{2} \gamma^{\tau j}
$$

a property called vanishing of Beta function, which implies that there exists $\lim _{j \rightarrow-\infty} \lambda_{j}$ and that this limit is an analytic function of $\lambda$. In its turn, the existence of this limit implies that there exist the limits

$$
\lim _{j \rightarrow-\infty} \log \frac{Z_{j-1}^{(2)}}{Z_{j}^{(2)}}=\eta_{2}(\lambda) \quad, \quad \lim _{k \rightarrow-\infty} \log \frac{Z_{j-1}}{Z_{j}}=\eta(\lambda),
$$

with $\eta(\lambda)=a_{2} \lambda^{2}+O\left(\lambda^{3}\right)$ and $\eta_{1}(\lambda)=a_{2} \lambda^{2}+O\left(\lambda^{3}\right), a_{2}>0$.

\subsection{The Dyson equation.}

Let us now prove the bound (3.2). We define

$$
\begin{gathered}
G_{\omega}^{4,1}\left(\mathbf{z}, \mathbf{x}_{1}, \mathbf{x}_{2}, \mathbf{x}_{3}, \mathbf{x}_{4}\right)=<\rho_{\mathbf{z}, \omega} ; \psi_{\mathbf{x}_{1},+}^{-} ; \psi_{\mathbf{x}_{2},+}^{+} ; \psi_{\mathbf{x}_{3},-}^{-} ; \psi_{\mathbf{x}_{4},-}^{+}>^{T} \\
G_{+}^{4}\left(\mathbf{x}_{1}, \mathbf{x}_{2}, \mathbf{x}_{3}, \mathbf{x}_{4}\right)=<\psi_{\mathbf{x}_{1},+}^{-} \psi_{\mathbf{x}_{2},+}^{+} ; \psi_{\mathbf{x}_{3},-}^{-} \psi_{\mathbf{x}_{4},-}^{+}>^{T}
\end{gathered}
$$

where

$$
\rho_{\mathbf{x}, \omega}=\psi_{\mathbf{x}, \omega}^{+} \psi_{\mathbf{x}, \omega}^{-}
$$

Moreover, we shall denote by $\hat{G}_{+\omega}^{4,1}\left(\mathbf{p} ; \mathbf{k}_{1}, \mathbf{k}_{2}, \mathbf{k}_{3}, \mathbf{k}_{4}\right)$ and $\hat{G}_{+}^{4}\left(\mathbf{k}_{1}, \mathbf{k}_{2}, \mathbf{k}_{3}, \mathbf{k}_{4}\right)$ the corresponding Fourier transforms, deprived of the momentum conservation delta. Note that, as a consequence of (1.1), if the $\psi^{+}$momenta are interpreted as "ingoing momenta" in the usual graph pictures, then the $\psi^{-}$momenta are "outgoing momenta"; our definition of Fourier transform is such that even $\mathbf{p}$, the momentum associated with the $\rho$ field, is an ingoing momentum. Hence, the momentum conservation implies that $\mathbf{k}_{1}+\mathbf{k}_{3}=\mathbf{k}_{2}+\mathbf{k}_{4}+\mathbf{p}$, in the case of $\hat{G}_{\omega}^{4,1}\left(\mathbf{p} ; \mathbf{k}_{1}, \mathbf{k}_{2}, \mathbf{k}_{3}, \mathbf{k}_{4}\right)$ and $\mathbf{k}_{1}+\mathbf{k}_{3}=$ $\mathbf{k}_{2}+\mathbf{k}_{4}$ in the case of $\hat{G}_{+}^{4}\left(\mathbf{k}_{1}, \mathbf{k}_{2}, \mathbf{k}_{3}, \mathbf{k}_{4}\right)$.

It is possible to derive a Dyson equation which, combined with the Ward identity (4.9) of ref. [BM3], gives a relation between $G^{4}, G^{2}$ and $G^{2,1}$.

If $Z=\int P(d \psi) \exp \{-V(\psi)\}$ and $<\cdot>$ denotes the expectation with respect to $Z^{-1} \int P(d \psi)$ $\exp \{-V(\psi)\}$

$$
G_{+}^{4}\left(\mathbf{x}_{1}, \mathbf{x}_{2}, \mathbf{x}_{3}, \mathbf{x}_{4}\right)=<\psi_{\mathbf{x}_{1},+}^{-} \psi_{\mathbf{x}_{2},+}^{+} \psi_{\mathbf{x}_{3},-}^{-} \psi_{\mathbf{x}_{4},-}^{+}>-G_{+}^{2}\left(\mathbf{x}_{1}, \mathbf{x}_{2}\right) G_{-}^{2}\left(\mathbf{x}_{3}, \mathbf{x}_{4}\right),
$$

where we used the fact that $\left\langle\psi_{\mathbf{x}, \omega}^{-} \psi_{\mathbf{y},-\omega}^{+}\right\rangle=0$.

Let $g_{\omega}(\mathbf{x})$ be the free propagator, whose Fourier transform is $g_{\omega}(\mathbf{k})=\chi_{h, 0}(\mathbf{k}) /\left(-i k_{0}+\omega k\right)$. Then, we can write the above equation as

$$
\begin{aligned}
& G_{+}^{4}\left(\mathbf{x}_{1}, \mathbf{x}_{2}, \mathbf{x}_{3}, \mathbf{x}_{4}\right)=-\lambda \int d \mathbf{z} g_{-}\left(\mathbf{z}-\mathbf{x}_{4}\right)<\psi_{\mathbf{x}_{1},+}^{-} \psi_{\mathbf{x}_{2},+}^{+} \psi_{\mathbf{x}_{3},-}^{-} \psi_{\mathbf{z},-}^{+} \psi_{\mathbf{z},+}^{+} \psi_{\mathbf{z},+}^{-}>+ \\
& +\lambda G_{+}^{2}\left(\mathbf{x}_{1}, \mathbf{x}_{2}\right) \int d \mathbf{z} g_{-}\left(\mathbf{z}-\mathbf{x}_{4}\right)<\psi_{\mathbf{x}_{3},-}^{-} \psi_{\mathbf{z},-}^{+} \psi_{\mathbf{z},+}^{+} \psi_{\mathbf{z},+}^{-}>= \\
& =-\lambda \int d \mathbf{z} g_{-1}\left(\mathbf{z}-\mathbf{x}_{4}\right)<\left[\psi_{\mathbf{x}_{1},+}^{-} \psi_{\mathbf{x}_{2},+}^{+}\right] ;\left[\psi_{\mathbf{x}_{3},-}^{-} \psi_{\mathbf{z},-}^{+} \psi_{\mathbf{z},+}^{+} \psi_{\mathbf{z},+}^{-}\right]>^{T} .
\end{aligned}
$$


From (3.9) we get

$$
\begin{aligned}
& -G_{+}^{4}\left(\mathbf{x}_{1}, \mathbf{x}_{2}, \mathbf{x}_{3}, \mathbf{x}_{4}\right)=\lambda \int d \mathbf{z} g_{-}\left(\mathbf{z}-\mathbf{x}_{4}\right)<\psi_{\mathbf{x}_{1},+}^{-} ; \psi_{\mathbf{x}_{2},+}^{+} ; \rho_{\mathbf{z},+}>^{T}<\psi_{\mathbf{x}_{3},-}^{-} \psi_{\mathbf{z},-}^{+}>+ \\
& +\lambda \int d \mathbf{z} g_{-}\left(\mathbf{z}-\mathbf{x}_{4}\right)<\rho_{\mathbf{z},+} ; \psi_{\mathbf{x}_{1},+}^{-} ; \psi_{\mathbf{x}_{2},+}^{+} ; \psi_{\mathbf{x}_{3},-}^{-} ; \psi_{\mathbf{z},-}^{+}>^{T}+ \\
& +\lambda \int d \mathbf{z} g_{-}\left(\mathbf{z}-\mathbf{x}_{4}\right)<\psi_{\mathbf{x}_{1},+}^{-} ; \psi_{\mathbf{x}_{2},+}^{+} ; \psi_{\mathbf{x}_{3},-}^{-} ; \psi_{\mathbf{z},-}^{+}>^{T}<\rho_{\mathbf{z},+}>
\end{aligned}
$$

The last addend is vanishing, since $\left\langle\rho_{\mathbf{z}, \omega}\right\rangle=0$ by the propagator parity properties. In terms of Fourier transforms, we get the Dyson equation

$$
\begin{aligned}
-\hat{G}_{+}^{4}\left(\mathbf{k}_{1}, \mathbf{k}_{2}, \mathbf{k}_{3}, \mathbf{k}_{4}\right) & =\lambda \hat{g}_{-}\left(\mathbf{k}_{4}\right)\left[\hat{G}_{-}^{2}\left(\mathbf{k}_{3}\right) \hat{G}_{+}^{2,1}\left(\mathbf{k}_{1}-\mathbf{k}_{2}, \mathbf{k}_{1}, \mathbf{k}_{2}\right)+\right. \\
& \left.+\frac{1}{L \beta} \sum_{\mathbf{p}} G_{+}^{4,1}\left(\mathbf{p} ; \mathbf{k}_{1}, \mathbf{k}_{2}, \mathbf{k}_{3}, \mathbf{k}_{4}-\mathbf{p}\right)\right]
\end{aligned}
$$

see Fig. 3.

Let us now suppose that $\left|\mathbf{k}_{4}\right| \leq \gamma^{h}$; then the support properties of the propagators imply that $|\mathbf{p}| \leq \gamma+\gamma^{h} \leq 2 \gamma$, hence we can freely multiply $G_{+}^{4,1}$ in the r.h.s. of (3.11) by the compact support function $\chi_{0}\left(\gamma^{-j_{m}}|\mathbf{p}|\right)$, with $j_{m}=\left[1+\log _{\gamma} 2\right]+1, \chi_{0}$ being defined as in (1.4). It follows that (3.11) can be written as

$$
\begin{aligned}
& -\hat{G}_{+}^{4}\left(\mathbf{k}_{1}, \mathbf{k}_{2}, \mathbf{k}_{3}, \mathbf{k}_{4}\right)=\lambda \hat{g}_{-}\left(\mathbf{k}_{4}\right)\left[\hat{G}_{-}^{2}\left(\mathbf{k}_{3}\right) \hat{G}_{+}^{2,1}\left(\mathbf{k}_{1}-\mathbf{k}_{2}, \mathbf{k}_{1}, \mathbf{k}_{2}\right)+\right. \\
& \left.+\frac{1}{L \beta} \sum_{\mathbf{p}} \chi_{M}(\mathbf{p}) G_{+}^{4,1}\left(\mathbf{p} ; \mathbf{k}_{1}, \mathbf{k}_{2}, \mathbf{k}_{3}, \mathbf{k}_{4}-\mathbf{p}\right)+\frac{1}{L \beta} \sum_{\mathbf{p}} \tilde{\chi}_{M}(\mathbf{p}) G_{+}^{4,1}\left(\mathbf{p} ; \mathbf{k}_{1}, \mathbf{k}_{2}, \mathbf{k}_{3}, \mathbf{k}_{4}-\mathbf{p}\right)\right],
\end{aligned}
$$

where $\chi_{M}(\mathbf{p})$ is a compact support function vanishing for $|\mathbf{p}| \geq \gamma^{h+j_{m}-1}$ and

$$
\tilde{\chi}_{M}(\mathbf{p})=\sum_{h_{p}=h+j_{m}}^{j_{m}} f_{h_{p}}(\mathbf{p}) .
$$

Note that the decomposition of the $\mathbf{p}$ sum is done so that $\tilde{\chi}_{M}(\mathbf{p})=0$ if $|\mathbf{p}| \leq 2 \gamma^{h}$.

Remark. The l.h.s. of the identity (3.12) is, by (2.37), of order $\lambda_{h} \gamma^{-4 h} Z_{h}^{-2}$; if we can prove that the l.h.s. is proportional to $\lambda$ with essentially the same proportionality constants, we get that $\lambda_{h} \simeq \lambda$. This cannot be achieved if we simply use (2.35), (2.36) and the analogous bound for $\hat{G}^{4,1}$, given in Lemma A1.2 of [BM3]; for instance, by using (2.35) and (2.36), we see that the first addend in the r.h.s. of (3.12) is of size $\gamma^{-2 h} Z_{h}^{(2)} Z_{h}^{-2} \lambda\left[1+O\left(\bar{\lambda}_{h}^{2}\right)\right]$. We have to take into account some crucial cancellations in the perturbative expansion, and this will be done by expressing $\hat{G}^{2,1}$ and $\hat{G}^{4,1}$ in terms of other functional integrals by suitable Ward identities which at the end will allow us to prove (3.2).

\subsection{Ward identities.}

By doing in (1.5) the chiral Gauge transformation $\psi_{\mathbf{x},+}^{\sigma} \rightarrow e^{i \sigma \alpha_{\mathbf{x},+}} \psi_{\mathbf{x},+}^{\sigma}, \psi_{\mathbf{x},-}^{\sigma} \rightarrow \psi_{\mathbf{x},-}^{\sigma}$, one obtains, see [BM2], the Ward identity, see Fig. 4

$$
D_{+}(\mathbf{p}) G_{+}^{2,1}(\mathbf{p}, \mathbf{k}, \mathbf{q})=G_{+}^{2}(\mathbf{q})-G_{+}^{2}(\mathbf{k})+\Delta_{+}^{2,1}(\mathbf{p}, \mathbf{k}, \mathbf{q})
$$

with

$$
\Delta_{+}^{2,1}(\mathbf{p}, \mathbf{k}, \mathbf{q})=\frac{1}{\beta L} \sum_{\mathbf{k}} C_{+}(\mathbf{k}, \mathbf{k}-\mathbf{p})<\hat{\psi}_{\mathbf{k},+}^{+} \hat{\psi}_{\mathbf{k}-\mathbf{p},+}^{-} ; \hat{\psi}_{\mathbf{k},+}^{-} \hat{\psi}_{\mathbf{q},+}^{+}>^{T}
$$


and

$$
C_{\omega}\left(\mathbf{k}^{+}, \mathbf{k}^{-}\right)=\left[C_{h, 0}\left(\mathbf{k}^{-}\right)-1\right] D_{\omega}\left(\mathbf{k}^{-}\right)-\left[C_{h, 0}\left(\mathbf{k}^{+}\right)-1\right] D_{\omega}\left(\mathbf{k}^{+}\right) .
$$

In the same way, we get two other Ward identities

$$
\begin{aligned}
& D_{+}(\mathbf{p}) G_{+}^{4,1}\left(\mathbf{p}, \mathbf{k}_{1}, \mathbf{k}_{2}, \mathbf{k}_{3}, \mathbf{k}_{4}-\mathbf{p}\right)=G_{+}^{4}\left(\mathbf{k}_{1}-\mathbf{p}, \mathbf{k}_{2}, \mathbf{k}_{3}, \mathbf{k}_{4}-\mathbf{p}\right)- \\
& \quad-G_{+}^{4}\left(\mathbf{k}_{1}, \mathbf{k}_{2}+\mathbf{p}, \mathbf{k}_{3}, \mathbf{k}_{4}-\mathbf{p}\right)+\Delta_{+}^{4,1} \\
& \quad-(\mathbf{p}) G_{-}^{4,1}\left(\mathbf{p}, \mathbf{k}_{1}, \mathbf{k}_{2}, \mathbf{k}_{3}, \mathbf{k}_{4}-\mathbf{p}\right)=G_{+}^{4}\left(\mathbf{k}_{1}, \mathbf{k}_{2}, \mathbf{k}_{3}-\mathbf{p}, \mathbf{k}_{4}-\mathbf{p}\right)- \\
& \quad-G_{+}^{4}\left(\mathbf{k}_{1}, \mathbf{k}_{2}, \mathbf{k}_{3}, \mathbf{k}_{4}\right)+\Delta_{-}^{4,1},
\end{aligned}
$$

where $\Delta_{ \pm}^{4,1}$ is the "correction term"

$$
\Delta_{ \pm}^{4,1}\left(\mathbf{p}, \mathbf{k}_{1}, \mathbf{k}_{2}, \mathbf{k}_{3}\right)=\frac{1}{\beta L} \sum_{\mathbf{k}} C_{ \pm}(\mathbf{k}, \mathbf{k}-\mathbf{p})<\hat{\psi}_{\mathbf{k}, \pm}^{+} \hat{\psi}_{\mathbf{k}-\mathbf{p}, \pm}^{-} ; \hat{\psi}_{\mathbf{k}_{1},+}^{-} ; \hat{\psi}_{\mathbf{k}_{2},+}^{+} ; \hat{\psi}_{\mathbf{k}_{3},-}^{-} ; \hat{\psi}_{\mathbf{k}_{4}-\mathbf{p},-}^{+}>^{T}
$$

\subsection{Counterterms}

Eq. (3.17) can be written, by adding and subtracting suitable counterterms $\nu_{ \pm}$, to be fixed properly later, see Fig. 6

$$
\begin{aligned}
& \left(1-\nu_{+}\right) D_{+}(\mathbf{p}) \hat{G}_{+}^{4,1}\left(\mathbf{p}, \mathbf{k}_{1}, \mathbf{k}_{2}, \mathbf{k}_{3}, \mathbf{k}_{4}-\mathbf{p}\right)-\nu_{-} D_{-}(\mathbf{p}) \hat{G}_{-}^{4,1}\left(\mathbf{p}, \mathbf{k}_{1}, \mathbf{k}_{2}, \mathbf{k}_{3}, \mathbf{k}_{4}-\mathbf{p}\right) \\
& =\hat{G}_{+}^{4}\left(\mathbf{k}_{1}-\mathbf{p}, \mathbf{k}_{2}, \mathbf{k}_{3}, \mathbf{k}_{4}-\mathbf{p}\right)-\hat{G}_{+}^{4}\left(\mathbf{k}_{1}, \mathbf{k}_{2}+\mathbf{p}, \mathbf{k}_{3}, \mathbf{k}_{4}-\mathbf{p}\right)+H_{+}^{4,1}\left(\mathbf{p}, \mathbf{k}_{1}, \mathbf{k}_{2}, \mathbf{k}_{3}, \mathbf{k}_{4}-\mathbf{p}\right),
\end{aligned}
$$

where by definition

$$
\begin{gathered}
H_{+}^{4,1}\left(\mathbf{p}, \mathbf{k}_{1}, \mathbf{k}_{2}, \mathbf{k}_{3}, \mathbf{k}_{4}-\mathbf{p}\right)=\frac{1}{\beta L} \sum_{\mathbf{k}} C_{+}(\mathbf{k}, \mathbf{k}-\mathbf{p})<\hat{\psi}_{\mathbf{k},+}^{+} \hat{\psi}_{\mathbf{k}-\mathbf{p},+}^{-} ; \hat{\psi}_{\mathbf{k}_{1},+}^{-} ; \hat{\psi}_{\mathbf{k}_{2},+}^{+} ; \hat{\psi}_{\mathbf{k}_{3},-}^{-} ; \hat{\psi}_{\mathbf{k}_{4}-\mathbf{p},-}^{+}>^{T}- \\
-\frac{1}{\beta L} \sum_{\mathbf{k}} \sum_{\omega} \nu_{\omega} D_{\omega}(\mathbf{p})<\hat{\psi}_{\mathbf{k}, \omega}^{+} \hat{\psi}_{\mathbf{k}-\mathbf{p}, \omega}^{-} ; \hat{\psi}_{\mathbf{k}_{1},+}^{-} ; \hat{\psi}_{\mathbf{k}_{2},+}^{+} ; \hat{\psi}_{\mathbf{k}_{3},-}^{-} ; \hat{\psi}_{\mathbf{k}_{4}-\mathbf{p},-}^{+}>^{T}
\end{gathered}
$$

In the same way, eq. (3.18) can be written as

$$
\begin{aligned}
& \left(1-\nu_{-}^{\prime}\right) D_{-}(\mathbf{p}) \hat{G}_{-}^{4,1}\left(\mathbf{p}, \mathbf{k}_{1}, \mathbf{k}_{2}, \mathbf{k}_{3}, \mathbf{k}_{4}-\mathbf{p}\right)-\nu_{+}^{\prime} D_{+}(\mathbf{p}) \hat{G}_{+}^{4,1}\left(\mathbf{p}, \mathbf{k}_{1}, \mathbf{k}_{2}, \mathbf{k}_{3}, \mathbf{k}_{4}-\mathbf{p}\right)= \\
& =\hat{G}_{+}^{4}\left(\mathbf{k}_{1}, \mathbf{k}_{2} \mathbf{k}_{3}-\mathbf{p}, \mathbf{k}_{4}-\mathbf{p}\right)-\hat{G}_{+}^{4}\left(\mathbf{k}_{1}, \mathbf{k}_{2}, \mathbf{k}_{3}, \mathbf{k}_{4}\right)+\hat{H}_{-}^{4,1}\left(\mathbf{p}, \mathbf{k}_{1}, \mathbf{k}_{2}, \mathbf{k}_{3}, \mathbf{k}_{4}-\mathbf{p}\right)
\end{aligned}
$$

where

$$
\begin{gathered}
H_{-}^{4,1}\left(\mathbf{p}, \mathbf{k}_{1}, \mathbf{k}_{2}, \mathbf{k}_{3}, \mathbf{k}_{4}-\mathbf{p}\right)=\frac{1}{\beta L} \sum_{\mathbf{k}} C_{-}(\mathbf{k}, \mathbf{k}-\mathbf{p})<\hat{\psi}_{\mathbf{k},-}^{+} \hat{\psi}_{\mathbf{k}-\mathbf{p},-}^{-} ; \hat{\psi}_{\mathbf{k}_{1},+}^{-} ; \hat{\psi}_{\mathbf{k}_{2},+}^{+} ; \hat{\psi}_{\mathbf{k}_{3},-}^{-} ; \hat{\psi}_{\mathbf{k}_{4}-\mathbf{p},-}^{+}>^{T}- \\
-\frac{1}{\beta L} \sum_{\mathbf{k}} \sum_{\omega} \nu_{\omega}^{\prime} D_{\omega}(\mathbf{p})<\hat{\psi}_{\mathbf{k}, \omega}^{+} \hat{\psi}_{\mathbf{k}-\mathbf{p}, \omega}^{-} ; \hat{\psi}_{\mathbf{k}_{1},+}^{-} ; \hat{\psi}_{\mathbf{k}_{2},+}^{+} ; \hat{\psi}_{\mathbf{k}_{3},-}^{-} ; \hat{\psi}_{\mathbf{k}_{4}-\mathbf{p},-}^{+}>^{T}
\end{gathered}
$$

If we insert in the r.h.s. of (3.20) the value of $\hat{G}_{-}^{4,1}$ taken from (3.22), we get

$$
\begin{aligned}
& (1+A) D_{+}(\mathbf{p}) \hat{G}_{+}^{4,1}\left(\mathbf{p}, \mathbf{k}_{1}, \mathbf{k}_{2}, \mathbf{k}_{3}, \mathbf{k}_{4}-\mathbf{p}\right)=\left[\hat{G}_{+}^{4}\left(\mathbf{k}_{1}-\mathbf{p}, \mathbf{k}_{2}, \mathbf{k}_{3}, \mathbf{k}_{4}-\mathbf{p}\right)-\right. \\
& \left.\hat{G}_{+}^{4}\left(\mathbf{k}_{1}, \mathbf{k}_{2}+\mathbf{p}, \mathbf{k}_{3}, \mathbf{k}_{4}-\mathbf{p}\right)\right]+B\left[\hat{G}_{+}^{4}\left(\mathbf{k}_{1}, \mathbf{k}_{3}-\mathbf{p}, \mathbf{k}_{4}-\mathbf{p}\right)-\hat{G}_{+}^{4}\left(\mathbf{k}_{1}, \mathbf{k}_{2}, \mathbf{k}_{3}, \mathbf{k}_{4}\right)\right]+\hat{H}_{+}^{4,1}+B \hat{H}_{-}^{4,1}
\end{aligned}
$$

where

$$
A=-\nu_{+}-\frac{\nu_{-} \nu_{+}^{\prime}}{1-\nu_{-}^{\prime}} \quad, \quad B=\frac{\nu_{-}}{1-\nu_{-}^{\prime}}
$$


If we insert in the last term of the r.h.s. of (3.12) the value of $\hat{G}_{+}^{4,1}$ taken from (3.24), we get

$$
\begin{aligned}
& -\hat{G}_{+}^{4}\left(\mathbf{k}_{1}, \mathbf{k}_{2}, \mathbf{k}_{3}, \mathbf{k}_{4}\right)= \\
& =\lambda \hat{g}_{-}\left(\mathbf{k}_{4}\right)\left[\hat{G}_{-}^{2}\left(\mathbf{k}_{3}\right) \hat{G}_{+}^{2,1}\left(\mathbf{k}_{1}-\mathbf{k}_{2}, \mathbf{k}_{1}, \mathbf{k}_{2}\right)+\frac{1}{L \beta} \sum_{\mathbf{p}} \chi_{M}(\mathbf{p}) G_{+}^{4,1}\left(\mathbf{p} ; \mathbf{k}_{1}, \mathbf{k}_{2}, \mathbf{k}_{3}, \mathbf{k}_{4}-\mathbf{p}\right)\right]+ \\
& +\frac{\lambda \hat{g}_{-}\left(\mathbf{k}_{4}\right)}{(1+A)} \frac{1}{L \beta} \sum_{\mathbf{p}} \tilde{\chi}_{M}(\mathbf{p}) \frac{\hat{G}_{+}^{4}\left(\mathbf{k}_{1}-\mathbf{p}, \mathbf{k}_{2}, \mathbf{k}_{3}, \mathbf{k}_{4}-\mathbf{p}\right)-\hat{G}_{+}^{4}\left(\mathbf{k}_{1}, \mathbf{k}_{2}+\mathbf{p}, \mathbf{k}_{3}, \mathbf{k}_{4}-\mathbf{p}\right)}{D_{+}(\mathbf{p})}+ \\
& +\frac{\lambda \hat{g}_{-}\left(\mathbf{k}_{4}\right)}{(1+A)} \frac{1}{L \beta} \sum_{\mathbf{p}} \tilde{\chi}_{M}(\mathbf{p}) \frac{\hat{G}_{+}^{4}\left(\mathbf{k}_{1}, \mathbf{k}_{2}, \mathbf{k}_{3}-\mathbf{p}, \mathbf{k}_{4}-\mathbf{p}\right)-\hat{G}_{+}^{4}\left(\mathbf{k}_{1}, \mathbf{k}_{2}, \mathbf{k}_{3}, \mathbf{k}_{4}\right)}{D_{+}(\mathbf{p})}+ \\
& +\frac{\lambda \hat{g}_{-}\left(\mathbf{k}_{4}\right)}{(1+A)} \frac{1}{L \beta} \sum_{\mathbf{p}} \tilde{\chi}_{M}(\mathbf{p}) \frac{\hat{H}_{+}^{4,1}\left(\mathbf{p} ; \mathbf{k}_{1}, \mathbf{k}_{2}, \mathbf{k}_{3}, \mathbf{k}_{4}-\mathbf{p}\right)+B \hat{H}_{-}^{4,1}\left(\mathbf{p} ; \mathbf{k}_{1}, \mathbf{k}_{2}, \mathbf{k}_{3}, \mathbf{k}_{4}-\mathbf{p}\right)}{D_{+}(\mathbf{p})}
\end{aligned}
$$

Note that

$$
\frac{1}{L \beta} \sum_{\mathbf{p}} \tilde{\chi}_{M}(\mathbf{p}) \frac{\hat{G}_{+}^{4}\left(\mathbf{k}_{1}, \mathbf{k}_{2}, \mathbf{k}_{3}, \mathbf{k}_{4}\right)}{D_{+}(\mathbf{p})}=0,
$$

since $D_{+}(\mathbf{p})$ is odd. Then, by using also the Ward identity (3.14), we get, if

$$
\mathbf{k}_{i}=\overline{\mathbf{k}}_{i} \quad, \quad \overline{\mathbf{k}}_{1}=\overline{\mathbf{k}}_{4}=-\overline{\mathbf{k}}_{2}=-\overline{\mathbf{k}}_{3}=\overline{\mathbf{k}} \quad, \quad|\overline{\mathbf{k}}|=\gamma^{h},
$$

the identity

$$
\begin{aligned}
& -\hat{G}_{+}^{4}\left(\overline{\mathbf{k}}_{1}, \overline{\mathbf{k}}_{2}, \overline{\mathbf{k}}_{3}, \overline{\mathbf{k}}_{4}\right)=\lambda \hat{g}_{-}\left(\overline{\mathbf{k}}_{4}\right) \hat{G}_{-}^{2}\left(\overline{\mathbf{k}}_{3}\right) \frac{G_{+}^{2}\left(\overline{\mathbf{k}}_{2}\right)-G_{+}^{2}\left(\overline{\mathbf{k}}_{1}\right)}{D_{+}(2 \overline{\mathbf{k}})}+ \\
& +\lambda \hat{g}_{-}\left(\overline{\mathbf{k}}_{4}\right) \hat{G}_{-}^{2}\left(\overline{\mathbf{k}}_{3}\right) \frac{\Delta_{+}^{2,1}\left(2 \overline{\mathbf{k}}, \overline{\mathbf{k}}_{1}, \overline{\mathbf{k}}_{2}\right)}{D_{+}(2 \overline{\mathbf{k}})}+\lambda \hat{g}_{-}\left(\overline{\mathbf{k}}_{4}\right) \frac{1}{L \beta} \sum_{\mathbf{p}} \chi_{M}(\mathbf{p}) G_{+}^{4,1}\left(\mathbf{p} ; \overline{\mathbf{k}}_{1}, \overline{\mathbf{k}}_{2}, \overline{\mathbf{k}}_{3}, \overline{\mathbf{k}}_{4}-\mathbf{p}\right)+ \\
& +\frac{\lambda \hat{g}_{-}\left(\overline{\mathbf{k}}_{4}\right)}{(1+A)} \frac{1}{L \beta} \sum_{\mathbf{p}} \tilde{\chi}_{M}(\mathbf{p}) \frac{\hat{G}_{+}^{4}\left(\overline{\mathbf{k}}_{1}-\mathbf{p}, \overline{\mathbf{k}}_{2}, \overline{\mathbf{k}}_{3}, \overline{\mathbf{k}}_{4}-\mathbf{p}\right)-\hat{G}_{+}^{4}\left(\overline{\mathbf{k}}_{1}, \overline{\mathbf{k}}_{2}+\mathbf{p}, \overline{\mathbf{k}}_{3}, \overline{\mathbf{k}}_{4}-\mathbf{p}\right)}{D_{+}(\mathbf{p})}+ \\
& +\frac{\lambda \hat{g}_{-}\left(\overline{\mathbf{k}}_{4}\right)}{(1+A)} \frac{1}{L \beta} \sum_{\mathbf{p}} \tilde{\chi}_{M}(\mathbf{p}) \frac{\hat{G}_{+}^{4}\left(\overline{\mathbf{k}}_{1}, \overline{\mathbf{k}}_{2}, \overline{\mathbf{k}}_{3}-\mathbf{p}, \overline{\mathbf{k}}_{4}-\mathbf{p}\right)}{D_{+}(\mathbf{p})}+ \\
& +\frac{\lambda \hat{g}_{-}\left(\overline{\mathbf{k}}_{4}\right)}{(1+A)} \frac{1}{L \beta} \sum_{\mathbf{p}} \tilde{\chi}_{M}(\mathbf{p}) \frac{\hat{H}_{+}^{4,1}\left(\mathbf{p} ; \overline{\mathbf{k}}_{1}, \overline{\mathbf{k}}_{2}, \overline{\mathbf{k}}_{3}, \overline{\mathbf{k}}_{4}-\mathbf{p}\right)+B \hat{H}_{-}^{4,1}\left(\mathbf{p} ; \overline{\mathbf{k}}_{1}, \overline{\mathbf{k}}_{2}, \overline{\mathbf{k}}_{3}, \overline{\mathbf{k}}_{4}-\mathbf{p}\right)}{D_{+}(\mathbf{p})}
\end{aligned}
$$

All the terms appearing in the above equation can be expressed in terms of convergent tree expansions. The term in the l.h.s. of (3.29) is given, by (2.37), by

$$
\hat{G}_{+}^{4}\left(\overline{\mathbf{k}}_{1}, \overline{\mathbf{k}}_{2}, \overline{\mathbf{k}}_{3}, \overline{\mathbf{k}}_{4}\right)=\gamma^{-4 h} Z_{h}^{-2}\left[-\lambda_{h}+O\left(\bar{\lambda}_{h}^{2}\right)\right] .
$$

The two terms in the r.h.s., first line, are equal, by $(2.36)$, to $\gamma^{-4 h} Z_{h}^{-2}\left(\lambda+O\left(\bar{\lambda}_{h}^{2}\right)\right)$. The first term in the second line, by (2.36) and eq. (177) of [BM2], can be bounded as

$$
\left|\lambda \hat{g}_{-}\left(\overline{\mathbf{k}}_{4}\right) \hat{G}_{-}^{2}\left(\overline{\mathbf{k}}_{3}\right) \frac{\Delta_{+}^{2,1}\left(2 \overline{\mathbf{k}}, \overline{\mathbf{k}}_{1}, \overline{\mathbf{k}}_{2}\right)}{D_{+}(2 \overline{\mathbf{k}})}\right| \leq C \bar{\lambda}_{h}^{2} \frac{\gamma^{-4 h}}{Z_{h}^{2}},
$$

while the second term in the second line, by eq. (A1.11) of ref. [BM3], can be bounded as

$$
\left|\lambda \hat{g}_{-}\left(\overline{\mathbf{k}}_{4}\right) \frac{1}{L \beta} \sum_{\mathbf{p}} \chi_{M}(\mathbf{p}) G_{+}^{4,1}\left(\mathbf{p} ; \overline{\mathbf{k}}_{1}, \overline{\mathbf{k}}_{2}, \overline{\mathbf{k}}_{3}, \overline{\mathbf{k}}_{4}-\mathbf{p}\right)\right| \leq C \bar{\lambda}_{h}^{3} \frac{\gamma^{-4 h}}{Z_{h}^{2}}
$$


Moreover, by using Lemma A1.1 of [BM3], one sees that

$$
\begin{aligned}
& \mid \frac{1}{L \beta} \sum_{\mathbf{p}} \tilde{\chi}_{M}(\mathbf{p}) \frac{\hat{G}_{+}^{4}\left(\overline{\mathbf{k}}_{1}-\mathbf{p}, \overline{\mathbf{k}}_{2}, \overline{\mathbf{k}}_{3}, \overline{\mathbf{k}}_{4}-\mathbf{p}\right)-\hat{G}_{+}^{4}\left(\overline{\mathbf{k}}_{1}, \overline{\mathbf{k}}_{2}+\mathbf{p}, \overline{\mathbf{k}}_{3}, \overline{\mathbf{k}}_{4}-\mathbf{p}\right)}{D_{+}(\mathbf{p})}+ \\
& +\frac{1}{L \beta} \sum_{\mathbf{p}} \tilde{\chi}_{M}(\mathbf{p}) \frac{\hat{G}_{+}^{4}\left(\overline{\mathbf{k}}_{1}, \overline{\mathbf{k}}_{2}, \overline{\mathbf{k}}_{3}-\mathbf{p}, \overline{\mathbf{k}}_{4}-\mathbf{p}\right)}{D_{+}(\mathbf{p})} \mid \leq C \bar{\lambda}_{h} \frac{\gamma^{-3 h}}{Z_{h}^{2}} .
\end{aligned}
$$

In the following sections we will prove the following Lemma.

Lemma 3.1 There exists $\varepsilon_{1} \leq \varepsilon_{0}$ and four $\lambda$-functions $\nu_{+}, \nu_{-}, \nu_{+}^{\prime}, \nu_{-}^{\prime}$ of order $\lambda$ (uniformly in h), such that, if $\bar{\lambda}_{h} \leq \varepsilon_{1}$,

$$
\left|\lambda \hat{g}_{-}\left(\overline{\mathbf{k}}_{4}\right) \frac{1}{L \beta} \sum_{\mathbf{p}} \tilde{\chi}_{M}(\mathbf{p}) \frac{\hat{H}_{ \pm}^{4,1}\left(\mathbf{p} ; \overline{\mathbf{k}}_{1}, \overline{\mathbf{k}}_{2}, \overline{\mathbf{k}}_{3}, \overline{\mathbf{k}}_{4}-\mathbf{p}\right)}{D_{+}(\mathbf{p})}\right| \leq C \bar{\lambda}_{h}^{2} \frac{\gamma^{-4 h}}{Z_{h}^{2}}
$$

The above Lemma, together with the identity (3.29), following from the Dyson equation and the Ward identities, and the previous bounds, proved in refs. [BM2] and [BM3] and following from the tree expansion, implies (3.2); this concludes the proof of Theorem 3.1.

\section{Proof of Lemma 3.1}

4.1 The corrections.

We shall prove first the bound (3.34) for $H_{+}^{4,1}$; the bound for $H_{-}^{4,1}$ is done essentially in the same way and will be briefly discussed later. By using (3.21), we get

$$
\begin{gathered}
\hat{g}_{-}\left(\mathbf{k}_{4}\right) \frac{1}{L \beta} \sum_{\mathbf{p}} \tilde{\chi}_{M}(\mathbf{p}) D_{+}^{-1}(\mathbf{p}) \hat{H}_{+}^{4,1}\left(\mathbf{p} ; \mathbf{k}_{1}, \mathbf{k}_{2}, \mathbf{k}_{3}, \mathbf{k}_{4}-\mathbf{p}\right)= \\
=\hat{g}_{-}\left(\mathbf{k}_{4}\right) \frac{1}{L \beta} \sum_{\mathbf{p}} \tilde{\chi}_{M}(\mathbf{p}) \frac{1}{L \beta} \sum_{\mathbf{k}} \frac{C_{+}(\mathbf{k}, \mathbf{k}-\mathbf{p})}{D_{+}(\mathbf{p})}<\hat{\psi}_{\mathbf{k},+}^{+} \hat{\psi}_{\mathbf{k}-\mathbf{p},+}^{-} ; \hat{\psi}_{\mathbf{k}_{1},+}^{-} ; \hat{\psi}_{\mathbf{k}_{2},+}^{+} ; \hat{\psi}_{\mathbf{k}_{3},-}^{-} ; \hat{\psi}_{\mathbf{k}_{4}-\mathbf{p},-}^{+}>^{T}+ \\
-\nu_{-} \hat{g}_{-}\left(\mathbf{k}_{4}\right) \frac{1}{L \beta} \sum_{\mathbf{p}} \tilde{\chi}_{M}(\mathbf{p}) \frac{1}{L \beta} \sum_{\mathbf{k}} \frac{D_{-}(\mathbf{p})}{D_{+}(\mathbf{p})}<\hat{\psi}_{\mathbf{k},-}^{+} \hat{\psi}_{\mathbf{k}-\mathbf{p},-}^{-} ; \hat{\psi}_{\mathbf{k}_{1},+}^{-} ; \hat{\psi}_{\mathbf{k}_{2},+}^{+} ; \hat{\psi}_{\mathbf{k}_{3},+}^{-} ; \hat{\psi}_{\mathbf{k}_{4}-\mathbf{p},-}^{+}>^{T}- \\
-\nu_{+} \hat{g}_{-}\left(\mathbf{k}_{4}\right) \frac{1}{L \beta} \sum_{\mathbf{p}} \tilde{\chi}_{M}(\mathbf{p}) \frac{1}{L \beta} \sum_{\mathbf{k}}<\hat{\psi}_{\mathbf{k},+}^{+} \hat{\psi}_{\mathbf{k}-\mathbf{p},+}^{-} ; \hat{\psi}_{\mathbf{k}_{1},+}^{-} ; \hat{\psi}_{\mathbf{k}_{2},+}^{+} ; \hat{\psi}_{\mathbf{k}_{3},-}^{-} ; \hat{\psi}_{\mathbf{k}_{4}-\mathbf{p},-}^{+}>^{T} .
\end{gathered}
$$

Let us define

$$
\tilde{G}_{+}^{4}\left(\mathbf{k}_{1}, \mathbf{k}_{2}, \mathbf{k}_{3}, \mathbf{k}_{4}\right)=\left.\frac{\partial^{4}}{\partial \phi_{\mathbf{k}_{1},+}^{+} \partial \phi_{\mathbf{k}_{2},+}^{-} \partial \phi_{\mathbf{k}_{3},-}^{+} \partial J_{\mathbf{k}_{4}}} \tilde{W}\right|_{\phi=0},
$$

where

$$
\begin{gathered}
\tilde{W}=\log \int P(d \hat{\psi}) e^{-T_{1}(\psi)+\nu_{+} T_{+}(\psi)+\nu_{-} T_{-}(\psi)} e^{-V(\hat{\psi})+\sum_{\omega} \int d \mathbf{x}\left[\phi_{\mathbf{x}, \omega}^{+} \hat{\psi}_{\mathbf{x}, \omega}^{-}+\hat{\psi}_{\mathbf{x}, \omega}^{+} \phi_{\mathbf{x}, \omega}^{-}\right]} \\
T_{1}(\psi)=\frac{1}{L \beta} \sum_{\mathbf{p}} \tilde{\chi}_{M}(\mathbf{p}) \frac{1}{L \beta} \sum_{\mathbf{k}} \frac{C_{+}(\mathbf{k}, \mathbf{k}-\mathbf{p})}{D_{+}(\mathbf{p})}\left(\hat{\psi}_{\mathbf{k},+}^{+} \hat{\psi}_{\mathbf{k}-\mathbf{p},+}^{-}\right) \hat{\psi}_{\mathbf{k}_{4}-\mathbf{p},-}^{+} \hat{J}_{\mathbf{k}_{4}} \hat{g}_{-}\left(\mathbf{k}_{4}\right) \\
T_{+}(\psi)=\frac{1}{L \beta} \sum_{\mathbf{p}} \tilde{\chi}_{M}(\mathbf{p}) \frac{1}{L \beta} \sum_{\mathbf{k}}\left(\hat{\psi}_{\mathbf{k},+}^{+} \hat{\psi}_{\mathbf{k}-\mathbf{p},+}^{-}\right) \hat{\psi}_{\mathbf{k}_{4}-\mathbf{p},-}^{+} \hat{J}_{\mathbf{k}_{4}} \hat{g}_{-}\left(\mathbf{k}_{4}\right)
\end{gathered}
$$


$T_{-}(\psi)=\frac{1}{L \beta} \sum_{\mathbf{p}} \tilde{\chi}_{M}(\mathbf{p}) \frac{1}{L \beta} \sum_{\mathbf{k}} \frac{D_{-}(\mathbf{p})}{D_{+}(\mathbf{p})}\left(\hat{\psi}_{\mathbf{k},-}^{+} \hat{\psi}_{\mathbf{k}-\mathbf{p},-}^{-}\right) \hat{\psi}_{\mathbf{k}_{4}-\mathbf{p},-}^{+} \hat{J}_{\mathbf{k}_{4}} \hat{g}_{-}\left(\mathbf{k}_{4}\right)$

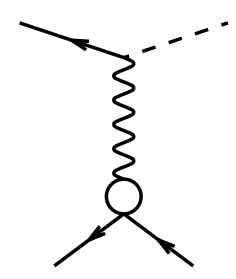

$T_{1}$

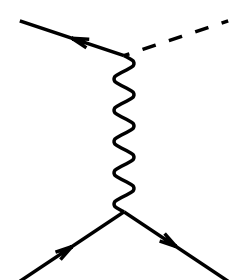

$T_{+}$

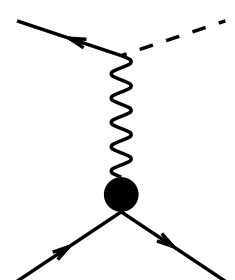

$T_{-}$

Fig. 12: Graphical representation of $T_{1}, T_{+}, T_{-}$; the dotted line carries momentum $\overline{\mathbf{k}}_{4}$, the empty circle represents $C_{+}$, the filled one $D_{-}(\mathbf{p}) / D_{+}(\mathbf{p})$

It is easy to see that $\tilde{G}_{+}^{4}$ is related to (4.1) by an identity similar to (3.9). In fact we can write

$$
\begin{aligned}
& -\tilde{G}_{+}^{4}\left(\mathbf{k}_{1}, \mathbf{k}_{2}, \mathbf{k}_{3}, \mathbf{k}_{4}\right)= \\
& =\hat{g}_{-}\left(\mathbf{k}_{4}\right) \frac{1}{L \beta} \sum_{\mathbf{p}} \tilde{\chi}_{M}(\mathbf{p}) \frac{1}{L \beta} \sum_{\mathbf{k}} \frac{C_{+}(\mathbf{k}, \mathbf{k}-\mathbf{p})}{D_{+}(\mathbf{p})}<\left[\hat{\psi}_{\mathbf{k}_{1},+}^{-} \hat{\psi}_{\mathbf{k}_{2},+}^{+}\right] ;\left[\hat{\psi}_{\mathbf{k},+}^{+} \hat{\psi}_{\mathbf{k}-\mathbf{p},+}^{-} \hat{\psi}_{\mathbf{k}_{3},-}^{-} \hat{\psi}_{\mathbf{k}_{4}-\mathbf{p},-}^{+}\right]>^{T}+ \\
& -\nu_{-} \hat{g}_{-}\left(\mathbf{k}_{4}\right) \frac{1}{L \beta} \sum_{\mathbf{p}} \tilde{\chi}_{M}(\mathbf{p}) \frac{1}{L \beta} \sum_{\mathbf{k}} \frac{D_{-}(\mathbf{p})}{D_{+}(\mathbf{p})}<\left[\hat{\psi}_{\mathbf{k}_{1},+}^{-} \hat{\psi}_{\mathbf{k}_{2},+}^{+}\right] ;\left[\hat{\psi}_{\mathbf{k},-}^{+} \hat{\psi}_{\mathbf{k}-\mathbf{p},-}^{-} \hat{\psi}_{\mathbf{k}_{3},+}^{-} \hat{\psi}_{\mathbf{k}_{4}-\mathbf{p},-}^{+}\right]>^{T}- \\
& -\nu_{+} \hat{g}_{-}\left(\mathbf{k}_{4}\right) \frac{1}{L \beta} \sum_{\mathbf{p}} \tilde{\chi}_{M}(\mathbf{p}) \frac{1}{L \beta} \sum_{\mathbf{k}}<\left[\hat{\psi}_{\mathbf{k}_{1},+}^{-} \hat{\psi}_{\mathbf{k}_{2},+}^{+}\right] ;\left[\hat{\psi}_{\mathbf{k},+}^{+} \hat{\psi}_{\mathbf{k}-\mathbf{p},+}^{-} \hat{\psi}_{\mathbf{k}_{3},-}^{-} \hat{\psi}_{\mathbf{k}_{4}-\mathbf{p},-}^{+}\right]>^{T} ;
\end{aligned}
$$

moreover, if we introduce the definition

$$
\delta \rho_{\mathbf{p},+}=\frac{1}{\beta L} \sum_{\mathbf{k}} \frac{C_{+}(\mathbf{p}, \mathbf{k})}{D_{+}(\mathbf{p})}\left(\hat{\psi}_{\mathbf{k},+}^{+} \hat{\psi}_{\mathbf{k}-\mathbf{p},+}^{-}\right),
$$

the term in the second line of (4.7) can be rewritten as

$$
\begin{aligned}
& \hat{g}_{-}\left(\mathbf{k}_{4}\right) \frac{1}{L \beta} \sum_{\mathbf{p}} \tilde{\chi}_{M}(\mathbf{p})<\left[\hat{\psi}_{\mathbf{k}_{1},+}^{-} \hat{\psi}_{\mathbf{k}_{2},+}^{+}\right] ;\left[\delta \rho_{\mathbf{p},+} \hat{\psi}_{\mathbf{k}_{3},-}^{-} \hat{\psi}_{\mathbf{k}_{4}-\mathbf{p},-}^{+}\right]>^{T}= \\
& =g_{-}\left(\mathbf{k}_{4}\right) \frac{1}{L \beta} \sum_{\mathbf{p}} \tilde{\chi}_{M}(\mathbf{p})\left[<\hat{\psi}_{\mathbf{k}_{1},+}^{-} ; \hat{\psi}_{\mathbf{k}_{2},+}^{+} ; \delta \rho_{\mathbf{p},+}>^{T}<\hat{\psi}_{\mathbf{k}_{3},-}^{-} \hat{\psi}_{\mathbf{k}_{4}-\mathbf{p},-}^{+}>+\right. \\
& \left.+<\delta \rho_{\mathbf{p},+} ; \hat{\psi}_{\mathbf{k}_{1,+}}^{-} ; \hat{\psi}_{\mathbf{k}_{2},+}^{+} ; \hat{\psi}_{\mathbf{k}_{3},-}^{-} ; \hat{\psi}_{\mathbf{k}_{4}-\mathbf{p},-}^{+}>^{T}\right],
\end{aligned}
$$

where we used the fact that $\mathbf{p} \neq 0$ in the support of $\tilde{\chi}_{M}(\mathbf{p})$ and $\left\langle\delta \rho_{\mathbf{p},+}>=0\right.$ for $\mathbf{p} \neq 0$. A similar decomposition can be done for the other two terms in the r.h.s. of (4.7); hence, by using (3.21), we get

$$
\begin{aligned}
& -\tilde{G}_{+}^{4}\left(\mathbf{k}_{1}, \mathbf{k}_{2}, \mathbf{k}_{3}, \mathbf{k}_{4}\right)=g_{-}\left(\mathbf{k}_{4}\right) \frac{1}{L \beta} \sum_{\mathbf{p}} \tilde{\chi}_{M}(\mathbf{p}) \frac{H_{+}^{4,1}\left(\mathbf{p} ; \mathbf{k}_{1}, \mathbf{k}_{2}, \mathbf{k}_{3}, \mathbf{k}_{4}-\mathbf{p}\right)}{D_{+}(\mathbf{p})}+ \\
& +\tilde{\chi}_{M}\left(\mathbf{k}_{1}-\mathbf{k}_{2}\right) g_{-}\left(\mathbf{k}_{4}\right) G_{-}^{2}\left(\mathbf{k}_{3}\right)\left[<\hat{\psi}_{\mathbf{k}_{1},+}^{-} ; \hat{\psi}_{\mathbf{k}_{2},+}^{+} ; \delta \rho_{\mathbf{k}_{1}-\mathbf{k}_{2},+}>^{T}-\right. \\
& \left.-\nu_{+}<\hat{\psi}_{\mathbf{k}_{1},+}^{-} ; \hat{\psi}_{\mathbf{k}_{2},+}^{+} ; \rho_{\mathbf{k}_{1}-\mathbf{k}_{2},+}>^{T}-\nu_{-} \frac{D_{-}\left(\mathbf{k}_{1}-\mathbf{k}_{2}\right)}{D_{+}\left(\mathbf{k}_{1}-\mathbf{k}_{2}\right)}<\hat{\psi}_{\mathbf{k}_{1},+}^{-} ; \hat{\psi}_{\mathbf{k}_{2},+}^{+} ; \rho_{\mathbf{k}_{1}-\mathbf{k}_{2},-}>^{T}\right]
\end{aligned}
$$

We now put $\mathbf{k}_{i}=\overline{\mathbf{k}}_{i}$, see (3.28). Since $\left|\overline{\mathbf{k}}_{1}-\overline{\mathbf{k}}_{2}\right|=2 \gamma^{h}$, (3.13) implies that $\tilde{\chi}_{M}\left(\overline{\mathbf{k}}_{1}-\overline{\mathbf{k}}_{2}\right)=0$; hence we get

$$
-\tilde{G}_{+}^{4}\left(\mathbf{k}_{1}, \mathbf{k}_{2}, \mathbf{k}_{3}, \mathbf{k}_{4}\right)=g_{-}\left(\mathbf{k}_{4}\right) \frac{1}{L \beta} \sum_{\mathbf{p}} \tilde{\chi}_{M}(\mathbf{p}) \frac{H_{+}^{4,1}\left(\mathbf{p} ; \mathbf{k}_{1}, \mathbf{k}_{2}, \mathbf{k}_{3}, \mathbf{k}_{4}-\mathbf{p}\right)}{D_{+}(\mathbf{p})} .
$$


Remark. (4.11) says that the last line of the Dyson equation (3.29) can be written as a functional integral very similar to the one for $G_{+}^{4}$ (we are essentially proceding as in the derivation of the Dyson equation, in the opposite direction) except that the interaction $V$ (1.3) is replaced by $V+T_{1}-\nu_{+} T_{+}-\nu_{-} T_{-}$; we will evaluate it via a multiscale integration procedure similar to the one for $G_{+}^{4}$, and in the expansion additional running coupling constants will appear; the expansion is convergent again if such new running couplings will remain small uniformly in the infrared cutoff.

\subsection{The properties of $D_{\omega}(\mathbf{p})^{-1} C_{\omega}(\mathbf{k}, \mathbf{k}-\mathbf{p})$.}

We shall use some properties of the operator $D_{\omega}(\mathbf{p})^{-1} C_{\omega}(\mathbf{k}, \mathbf{k}-\mathbf{p})$, which were proved in [BM2]. Let us consider first the effect of contracting both $\hat{\psi}$ fields of $\delta \rho_{\mathbf{p},+}$ on the same or two different scales; in the second case, we also suppose that the regularization procedure (to be defined later, in agreement with this hypothesis) does not act on the propagator of higher scale. Hence, we have to study the quantity

$$
\Delta_{\omega}^{(i, j)}\left(\mathbf{k}^{+}, \mathbf{k}^{-}\right)=\frac{C_{\omega}\left(\mathbf{k}^{+}, \mathbf{k}^{-}\right)}{D_{\omega}(\mathbf{p})} \tilde{g}_{\omega}^{(i)}\left(\mathbf{k}^{+}\right) \tilde{g}_{\omega}^{(j)}\left(\mathbf{k}^{-}\right)
$$

where $\mathbf{p}=\mathbf{k}^{+}-\mathbf{k}^{-}$. The crucial observation is that

$$
\Delta_{\omega}^{(i, j)}\left(\mathbf{k}^{+}, \mathbf{k}^{-}\right)=0 \quad, \quad \text { if } h<i, j<0,
$$

since $\chi_{h, 0}\left(\mathbf{k}^{ \pm}\right)=1$, if $h<i, j<0$. Let us then consider the cases in which $\Delta_{\omega}^{(i, j)}\left(\mathbf{k}^{+}, \mathbf{k}^{-}\right)$is not identically equal to 0 . Since $\Delta_{\omega}^{(i, j)}\left(\mathbf{k}^{+}, \mathbf{k}^{-}\right)=\Delta_{\omega}^{(j, i)}\left(\mathbf{k}^{-}, \mathbf{k}^{+}\right)$, we can restrict the analysis to the case $i \geq j$. We define

$$
u_{0}(\mathbf{k})=\left\{\begin{array}{ll}
0 & \text { if }|\mathbf{k}| \leq 1 \\
1-f_{0}(\mathbf{k}) & \text { if } 1 \leq|\mathbf{k}|
\end{array} \quad, \quad u_{h}(\mathbf{k})=\left\{\begin{array}{ll}
0 & \text { if }|\mathbf{k}| \geq \gamma^{h} \\
1-f_{h}(\mathbf{k}) & \text { if }|\mathbf{k}| \leq \gamma^{h}
\end{array} .\right.\right.
$$

Then we get, by using (2.19), the fact that $Z_{-1}=Z_{0}=1$ and $\tilde{f}_{j}=f_{j}$ for $j=0, h$,

$$
\begin{gathered}
\Delta_{\omega}^{(0,0)}\left(\mathbf{k}^{+}, \mathbf{k}^{-}\right)=\frac{1}{D_{\omega}(\mathbf{p})}\left[\frac{f_{0}\left(\mathbf{k}^{+}\right)}{D_{\omega}\left(\mathbf{k}^{+}\right)} u_{0}\left(\mathbf{k}^{-}\right)-\frac{f_{0}\left(\mathbf{k}^{-}\right)}{D_{\omega}\left(\mathbf{k}^{-}\right)} u_{0}\left(\mathbf{k}^{+}\right)\right], \\
\Delta_{\omega}^{(h, h)}\left(\mathbf{k}^{+}, \mathbf{k}^{-}\right)=\frac{1}{D_{\omega}(\mathbf{p})} \frac{1}{\tilde{Z}_{h-1}\left(\mathbf{k}^{+}\right) \tilde{Z}_{h-1}\left(\mathbf{k}^{-}\right)}\left[\frac{f_{h}\left(\mathbf{k}^{+}\right) u_{h}\left(\mathbf{k}^{-}\right)}{D_{\omega}\left(\mathbf{k}^{+}\right)}-\frac{u_{h}\left(\mathbf{k}^{+}\right) f_{h}\left(\mathbf{k}^{-}\right)}{D_{\omega}\left(\mathbf{k}^{-}\right)}\right], \\
\Delta_{\omega}^{(0, h)}\left(\mathbf{k}^{+}, \mathbf{k}^{-}\right)=\frac{1}{D_{\omega}(\mathbf{p})} \frac{1}{\tilde{Z}_{h-1}\left(\mathbf{k}^{-}\right)}\left[\frac{f_{0}\left(\mathbf{k}^{+}\right) u_{h}\left(\mathbf{k}^{-}\right)}{D_{\omega}\left(\mathbf{k}^{+}\right)}-\frac{f_{h}\left(\mathbf{k}^{-}\right) u_{0}\left(\mathbf{k}^{+}\right)}{D_{\omega}\left(\mathbf{k}^{-}\right)}\right], \\
\Delta_{\omega}^{(0, j)}\left(\mathbf{k}^{+}, \mathbf{k}^{-}\right)=-\frac{1}{Z_{j-1}} \frac{\tilde{f}_{j}\left(\mathbf{k}^{-}\right) u_{0}\left(\mathbf{k}^{+}\right)}{D_{\omega}(\mathbf{p}) D_{\omega}\left(\mathbf{k}^{-}\right)} \quad, \quad h<j<0, \\
\Delta_{\omega}^{(i, h)}\left(\mathbf{k}^{+}, \mathbf{k}^{-}\right)=\frac{1}{Z_{h-1}\left(\mathbf{k}^{-}\right) Z_{i-1}} \frac{\tilde{f}_{i}\left(\mathbf{k}^{+}\right) u_{h}\left(\mathbf{k}^{-}\right)}{D_{\omega}(\mathbf{p}) D_{\omega}\left(\mathbf{k}^{+}\right)} \quad, \quad j=h<i \leq-1 .
\end{gathered}
$$

As an easy consequence of the above equations, as shown in $\S 4.2$ of [BM2], one can write, for $0 \geq j>h$,

$$
\Delta_{\omega}^{(0, j)}\left(\mathbf{k}^{+}, \mathbf{k}^{-}\right)=\frac{\mathbf{p}}{D_{\omega}(\mathbf{p})} \mathbf{S}_{\omega}^{(j)}\left(\mathbf{k}^{+}, \mathbf{k}^{-}\right),
$$

where $S_{\omega, i}^{(j)}\left(\mathbf{k}^{+}, \mathbf{k}^{-}\right)$are smooth functions such that

$$
\left|\partial_{\mathbf{k}^{+}}^{m_{+}} \partial_{\mathbf{k}^{-}}^{m_{j}} S_{\omega, i}^{(j)}\left(\mathbf{k}^{+}, \mathbf{k}^{-}\right)\right| \leq C_{m_{0}+m_{j}} \frac{\gamma^{-j\left(1+m_{j}\right)}}{Z_{j-1}}
$$


Finally, it is easy to see that, if $0>i \geq h$,

$$
\left|\Delta_{\omega}^{(i, h)}\left(\mathbf{k}^{+}, \mathbf{k}^{-}\right)\right| \leq C \gamma^{-(i-h)} \frac{\gamma^{-h-i}}{Z_{i-1}} .
$$

Note that, in the r.h.s. of (4.22), there is apparently a $Z_{h-1}^{-1}$ factor missing, but the bound can not be improved; this is a consequence of the fact that $\tilde{Z}_{h-1}(\mathbf{k})=0$ for $|\mathbf{k}| \leq \gamma^{h-1}$, see eq. (63) of [BM2], and the support properties of $u_{h}(\mathbf{k})$. In any case, this is not a problem, since the dimensional dependence of $\Delta^{(i, h)}$ on the field renormalization constants is exactly $Z^{-1}$. Note also the presence in the bound of the extra factor $\gamma^{-(i-h)}$, with respect to the dimensional bound; it will allow us to avoid renormalization of the marginal terms containing $\Delta^{(i, h)}$.

4.3 The multiscale expansion of $\tilde{G}_{+}^{4}\left(\overline{\mathbf{k}}_{1}, \overline{\mathbf{k}}_{2}, \overline{\mathbf{k}}_{3}, \overline{\mathbf{k}}_{4}\right)$ : the first integration step.

The calculation of $\tilde{G}_{+}^{4}$ is done via a multiscale expansion; we shall concentrate on the differences with respect to that described in $\S 2$, due to the presence in the potential of the terms $T_{1}(\psi)$ and $T_{ \pm}(\psi)$. Moreover we shall suppose that the momenta $\mathbf{k}_{i}$ are put equal to $\overline{\mathbf{k}}_{i}$, defined as in (3.28). Let us consider the first step of the iterative integration procedure, the integration of the field $\psi^{(0)}$; we shall describe only the terms linear in the external $J$ field, the only ones contributing to $\tilde{G}_{+}^{4}$ which were not already discussed. We call $\overline{\mathcal{V}}^{(-1)}\left(\psi^{[h,-1]}\right)$ the contribution to the effective potential of such terms and we write

$$
\overline{\mathcal{V}}^{(-1)}\left(\psi^{[h,-1]}\right)=\overline{\mathcal{V}}_{a, 1}^{(-1)}\left(\psi^{[h,-1]}\right)+\overline{\mathcal{V}}_{a, 2}^{(-1)}\left(\psi^{[h,-1]}\right)+\overline{\mathcal{V}}_{b, 1}^{(-1)}\left(\psi^{[h,-1]}\right)+\overline{\mathcal{V}}_{b, 2}^{(-1)}\left(\psi^{[h,-1]}\right),
$$

where $\overline{\mathcal{V}}_{a, 1}^{(-1)}+\overline{\mathcal{V}}_{a, 2}^{(-1)}$ is the sum of the terms in which the field $\hat{\psi}_{\mathbf{\mathbf { k }}_{4}-\mathbf{p},-}^{+}$appearing in the definition of $T_{1}(\psi)$ or $T_{ \pm}(\psi)$ is contracted, $\overline{\mathcal{V}}_{a, 1}^{(-1)}$ and $\overline{\mathcal{V}}_{a, 2}^{(-1)}$ denoting the sum over the terms of this type containing a $T_{1}$ or a $T_{ \pm}$vertex, respectively; $\overline{\mathcal{V}}_{b, 1}^{(-1)}+\overline{\mathcal{V}}_{b, 2}^{(-1)}$ is the sum of the other terms, that is those where the field $\hat{\psi}_{\overline{\mathbf{k}}_{4}-\mathbf{p},-}^{+}$is an external field, the index $i=1,2$ having the same meaning as before.

Note that the condition (3.28) on the external momenta $\mathbf{k}_{i}$ forbids the presence of vertices of type $\varphi$, if $h<0$, as we shall suppose. Hence, all graphs contributing to $\overline{\mathcal{V}}^{(-1)}$ have, besides the external field of type $J$, an odd number of external fields of type $\psi$.

Let us consider first $\overline{\mathcal{V}}_{a, 1}^{(-1)}$; we shall still distinguish different group of terms, those where both fields $\hat{\psi}_{\mathbf{k},+}^{+}$and $\hat{\psi}_{\mathbf{k}-\mathbf{p},+}^{-}$are contracted, those where only one among them is contracted and those where no one is contracted.

If no one of the fields $\hat{\psi}_{\mathbf{k},+}^{+}$and $\hat{\psi}_{\mathbf{k}-\mathbf{p},+}^{-}$is contracted, we can only have terms with at least four external lines; for the properties of $\Delta^{i j}$, at least one of the fields $\hat{\psi}_{\mathbf{k},+}^{+}$and $\hat{\psi}_{\mathbf{k}+\mathbf{p},+}^{-}$must be contracted at scale $h$. If one of these terms has four external lines, hence it is marginal, it has the following form

$$
\int d \mathbf{p} \tilde{\chi}_{M}(\mathbf{p}) \hat{\psi}_{-, \overline{\mathbf{k}}_{4}-\mathbf{p}}^{+} G_{2}^{(0)}\left(\overline{\mathbf{k}}_{4}-\mathbf{p}\right) \hat{g}_{-}^{(0)}\left(\overline{\mathbf{k}}_{4}-\mathbf{p}\right) \hat{g}_{-}\left(\overline{\mathbf{k}}_{4}\right) \hat{J}_{\overline{\mathbf{k}}_{4}} \int d \mathbf{k} \frac{C(\mathbf{k}, \mathbf{p})}{D_{+}(\mathbf{p})} \hat{\psi}_{\mathbf{k},+}^{+} \hat{\psi}_{\mathbf{k}+\mathbf{p},+}^{-},
$$

where $G_{2}^{(0)}(\mathbf{k})$ is a suitable function which can be expressed as a sum of graphs with an odd number of propagators, hence it vanishes at $\mathbf{k}=0$. This implies that $G_{2}^{(0)}(0)=0$, so that we can regularize it without introducing any running coupling.

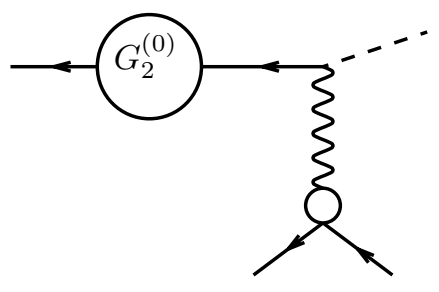


Fig. 13: Graphical representation of (4.24)

If both $\hat{\psi}_{\mathbf{k},+}^{+}$and $\hat{\psi}_{\mathbf{k}-\mathbf{p},+}^{-}$in $T_{1}(\psi)$ are contracted, we get terms of the form

$$
\tilde{W}_{n+1}^{(-1)}\left(\overline{\mathbf{k}}_{4}, \mathbf{k}_{1}, . ., \mathbf{k}_{n}\right) \hat{g}_{-}\left(\overline{\mathbf{k}}_{4}\right) \hat{J}_{\overline{\mathbf{k}}_{4}} \prod_{i=1}^{n} \hat{\psi}_{\mathbf{k}_{i}}^{\varepsilon_{i}},
$$

where $n$ is an odd integer. We want to define an $\mathcal{R}$ operation for such terms. There is apparently a problem, as the $\mathcal{R}$ operation involves derivatives and in $\tilde{W}^{(-1)}$ appears the function $\Delta^{(0,0)}$ of the form (4.20) and the cutoff function $\tilde{\chi}_{M}(\mathbf{p})$, with support on momenta of size $\gamma^{h}$. Hence one can worry about the derivatives of the factor $\tilde{\chi}_{M}(\mathbf{p}) \mathbf{p} D_{+}(\mathbf{p})^{-1}$. However, as the line of momentum $\overline{\mathbf{k}}_{4}-\mathbf{p}$ is necessarily at scale 0 (we are considering terms in which it is contracted), then $|\mathbf{p}| \geq \gamma^{-1}-\gamma^{h} \geq \gamma^{-1} / 2$ (for $|h|$ large enough), so that we can freely multiply by a smooth cutoff function $\bar{\chi}(\mathbf{p})$ restricting $\mathbf{p}$ to the allowed region; this allows us to pass to coordinate space and shows that the $\mathcal{R}$ operation can be defined in the usual way. We define

$$
\begin{gathered}
\mathcal{L} \tilde{W}_{4}^{(-1)}\left(\overline{\mathbf{k}}_{4}, \mathbf{k}_{1}, \mathbf{k}_{2}, \mathbf{k}_{3}\right)=\tilde{W}_{4}^{(-1)}(0, . ., 0) \\
\mathcal{L} \tilde{W}_{2}^{(-1)}\left(\overline{\mathbf{k}}_{4}\right)=\tilde{W}_{2}^{(-1)}(0)+\overline{\mathbf{k}}_{4} \partial_{\mathbf{k}} \tilde{W}_{2}^{(-1)}(0) .
\end{gathered}
$$

Note that by parity the first term in (4.27) is vanishing; this means that there are only marginal terms. Note also that the local term proportional to $\hat{J}_{\overline{\mathbf{k}}_{4}} \hat{\psi}_{\mathbf{\mathbf { k }}_{4},-}^{+}$is such that the field $\hat{\psi}_{\mathbf{k}_{4},-}^{+}$can be contracted only at the last scale $h$; hence it has any influence on the integrations of all the scales $>h$.
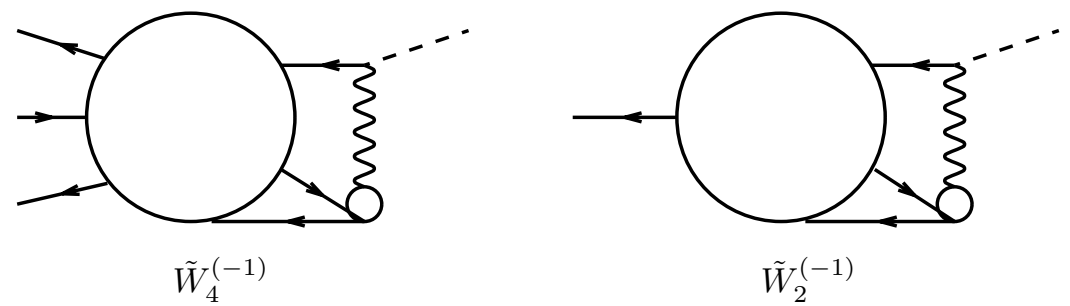

Fig. 14: Graphical representation of $\tilde{W}_{4}^{(-1)}$ and $\tilde{W}_{2}^{(-1)}$

If only one among the fields $\hat{\psi}_{\mathbf{k},+}^{+}$and $\hat{\psi}_{\mathbf{k}-\mathbf{p},+}^{-}$in $T_{1}(\psi)$ is contracted, we note first that we cannot have terms with two external lines (including $\hat{J}_{\mathbf{k}_{4}}$ ); in fact in such a case there is an external line with momentum $\overline{\mathbf{k}}_{4}$ with $\omega=-$ and the other has $\omega=+$; this is however forbidden by global gauge invariance. Moreover, for the same reasons as before, we do not have to worry about the derivatives of the factor $\tilde{\chi}_{M}(\mathbf{p}) \mathbf{p} D_{+}(\mathbf{p})^{-1}$, related with the regularization procedure of the terms with four external lines, which have the form

$$
\begin{aligned}
& \int d \mathbf{k}^{+} \hat{\psi}_{\mathbf{k}_{1},+}^{+} \hat{\psi}_{\mathbf{k}^{-},+}^{-} \hat{\psi}_{\mathbf{k}^{-}+\overline{\mathbf{k}}_{4}-\mathbf{k}_{1},-} \hat{g}_{-}\left(\overline{\mathbf{k}}_{4}\right) \hat{J}_{\overline{\mathbf{k}}_{4}} \tilde{\chi}_{M}\left(\mathbf{k}^{+}-\mathbf{k}^{-}\right) \hat{g}_{-}^{(0)}\left(\overline{\mathbf{k}}_{4}-\mathbf{k}^{+}+\mathbf{k}^{-}\right) \cdot \\
& \cdot G_{4}^{(0)}\left(\mathbf{k}^{+}, \overline{\mathbf{k}}_{4}, \mathbf{k}_{1}\right)\left\{\frac{\left[C_{h, 0}\left(\mathbf{k}^{-}\right)-1\right] D_{+}\left(\mathbf{k}^{-}\right) \hat{g}_{+}^{(0)}\left(\mathbf{k}^{+}\right)}{D_{+}\left(\mathbf{k}^{+}-\mathbf{k}^{-}\right)}-\frac{u_{0}\left(\mathbf{k}^{+}\right)}{D_{+}\left(\mathbf{k}^{+}-\mathbf{k}^{-}\right)}\right\},
\end{aligned}
$$

or the similar one with the roles of $\mathbf{k}^{+}$and $\mathbf{k}^{-}$exchanged.

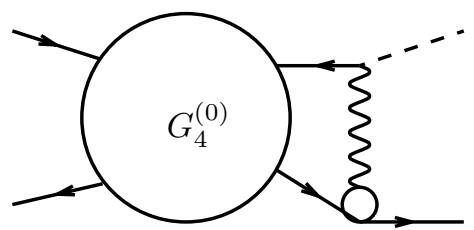


Fig. 15: Graphical representation of a single addend in (4.28)

The two terms in (4.28) must be treated differently, as concerns the regularization procedure. The first term is such that one of the external lines is associated with the operator $\left[C_{h, 0}\left(\mathbf{k}^{-}\right)-\right.$ $1] D_{+}\left(\mathbf{k}^{-}\right) D_{+}(\mathbf{p})^{-1}$. We define $\mathcal{R}=1$ for such terms; in fact, when such external line is contracted (and this can happen only at scale $h$ ), the factor $D_{+}\left(\mathbf{k}^{-}\right) D_{+}(\mathbf{p})^{-1}$ produces an extra factor $\gamma^{h}$ in the bound, with respect to the dimensional one. This claim simply follows by the observation that $\left|D_{+}(\mathbf{p})\right| \geq 1-\gamma^{-1}$ as $\mathbf{p}=\mathbf{k}^{+}-\mathbf{k}^{-}$and $\mathbf{k}^{+}$is at scale 0 , while $\mathbf{k}^{-}$, as we said, is at scale $h$. This factor has the effect that all the marginal terms in the tree path connecting $v_{0}$ with the end-point to which is associated the $T_{1}$ vertex acquires negative dimension.

The second term in (4.28) can be regularized as above, by subtracting the value of the kernel computed at zero external momenta, i.e. for $\mathbf{k}^{-}=\overline{\mathbf{k}}_{4}=\mathbf{k}_{1}=0$. Note that such local part is given by

$$
\int d \mathbf{k}^{+} \tilde{\chi}_{M}\left(\mathbf{k}^{+}\right) \hat{g}_{-}^{(0)}\left(\mathbf{k}^{+}\right) G_{2}^{(0)}\left(\mathbf{k}^{+}, 0,0\right) \frac{u_{0}\left(\mathbf{k}^{+}\right)}{D_{+}\left(\mathbf{k}^{+}\right)},
$$

and there is no singularity associated with the factor $D_{+}\left(\mathbf{k}^{+}\right)^{-1}$, thanks to the support on scale 0 of the propagator $\hat{g}_{-}^{(0)}\left(\mathbf{k}^{+}\right)$.

A similar (but simpler) analysis holds for the terms contributing to $\overline{\mathcal{V}}_{a, 2}^{(-1)}$, which contain a vertex of type $T_{+}$or $T_{-}$and are of order $\lambda \nu_{ \pm}$. Now, the only thing to analyze carefully is the possible singularities associated with the factors $\tilde{\chi}_{M}(\mathbf{p})$ and $\mathbf{p} D_{+}(\mathbf{p})^{-1}$. However, since in these terms the field $\hat{\psi}_{\overline{\mathbf{k}}_{4}-\mathbf{p},-}^{+}$is contracted, $|\mathbf{p}| \geq \gamma^{-1} / 2$, for $|h|$ large enough, a property already used before; hence the regularization procedure can not produce bad dimensional bounds.

We will define $\tilde{z}_{-1}$ and $\tilde{\lambda}_{-1}$, so that

$$
\mathcal{L}\left[\overline{\mathcal{V}}_{a, 1}^{(-1)}+\overline{\mathcal{V}}_{a, 2}^{(-1)}\right]\left(\psi^{[h,-1]}\right)=\left[\tilde{\lambda}_{-1} \bar{F}_{\lambda}^{[h,-1]}\left(\psi^{[h,-1]}\right)+\tilde{z}_{-1} \hat{\psi}_{\overline{\mathbf{k}}_{4},-}^{[h,-1]+} D_{-}\left(\overline{\mathbf{k}}_{4}\right)\right] \hat{g}_{-}\left(\overline{\mathbf{k}}_{4}\right) \hat{J}_{\overline{\mathbf{k}}_{4}},
$$

where we used the definition

$$
\bar{F}_{\lambda}^{[h, j]}\left(\psi^{[h, j]}\right)=\frac{1}{(L \beta)^{4}} \sum_{\mathbf{k}_{1}, \mathbf{k}_{2}, \mathbf{k}_{3}: C_{h, j}^{-1}\left(\mathbf{k}_{i}\right)>0} \hat{\psi}_{\mathbf{k}_{1},+}^{[h, j]+} \hat{\psi}_{\mathbf{k}_{2},+}^{[h, j]-} \hat{\psi}_{\mathbf{k}_{3},-}^{[h, j]+} \delta\left(\mathbf{k}_{1}-\mathbf{k}_{2}+\mathbf{k}_{3}-\overline{\mathbf{k}}_{4}\right) .
$$

Note that there is no first order contribution to $\tilde{\lambda}_{-1}$, as follows from a simple calculation, so that $\tilde{\lambda}_{-1}$ is of order $\lambda^{2}$ or lower. We expect indeed that it satisfies a non zero lower bound of order $\lambda^{2}$, but this will not play any role in the following.

Let us consider now the terms contributing to $\overline{\mathcal{V}}_{b, 1}^{(-1)}$, that is those where $\hat{\psi}_{\overline{\mathbf{k}}_{4}-\mathbf{p}}^{+}$is not contracted and there is a vertex of type $T_{1}$.

Besides the term of order 0 in $\lambda$ and $\nu_{ \pm}$, equal to $T_{1}\left(\psi^{[h,-1]}\right)$, there are the terms containing at least one vertex $\lambda$; among these terms, the only marginal ones (those requiring a regularization) have four external lines (including $\hat{J}_{\mathbf{k}_{4}}$ ), since the oddness of the propagator does not allow tadpoles. These terms are of the form

$$
\begin{aligned}
& \sum_{\tilde{\omega}} \int d \mathbf{p} \tilde{\chi}_{M}(\mathbf{p}) \hat{\psi}_{\mathbf{k}^{+}, \tilde{\omega}}^{+} \int d \mathbf{k}^{+} \hat{\psi}_{\mathbf{k}^{+}-\mathbf{p}, \tilde{\omega}}^{+} \hat{\psi}_{\mathbf{k}_{4}-\mathbf{p},-}^{+} \hat{g}_{-}\left(\overline{\mathbf{k}}_{4}\right) \hat{J}_{\overline{\mathbf{k}}_{4}} . \\
& {\left[F_{2,+, \tilde{\omega}}^{(-1)}\left(\mathbf{k}^{+}, \mathbf{k}^{+}-\mathbf{p}\right)+F_{1,+}^{(-1)}\left(\mathbf{k}^{+}, \mathbf{k}^{+}-\mathbf{p}\right) \delta_{+, \tilde{\omega}}\right]}
\end{aligned}
$$

where $F_{2,+, \tilde{\omega}}^{(-1)}$ and $F_{1,+}^{(-1)}$ are defined as in eq. (132) of [BM2]; they represent the terms in which both or only one of the fields in $\delta \rho_{\mathbf{p},+}$, respectively, are contracted. Both contributions to the r.h.s. of (4.32) are dimensionally marginal; however, the regularization of $F_{1,+}^{(-1)}$ is trivial, as it is of the form

$$
F_{1,+}^{(-1)}\left(\mathbf{k}^{+}, \mathbf{k}^{-}\right)=\left[\frac{\left[C_{h, 0}\left(\mathbf{k}^{-}\right)-1\right] D_{+}\left(\mathbf{k}^{-}\right) \hat{g}_{+}^{(0)}\left(\mathbf{k}^{+}\right)-u_{0}\left(\mathbf{k}^{+}\right)}{D_{+}\left(\mathbf{k}^{+}-\mathbf{k}^{-}\right)} G^{(2)}\left(\mathbf{k}^{+}\right)\right.
$$


or the similar one, obtained exchanging $\mathbf{k}^{+}$with $\mathbf{k}^{-}$.

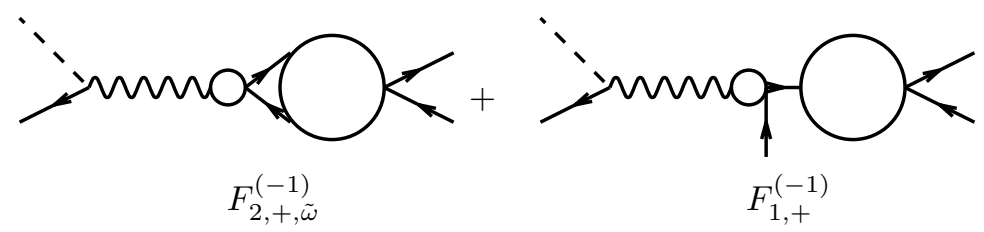

Fig. 16: Graphical representation of (4.33)

By the oddness of the propagator in the momentum, $G^{(2)}(0)=0$, hence we can regularize such term without introducing any local term, by simply rewriting it as

$$
F_{1,+}^{(-1)}\left(\mathbf{k}^{+}, \mathbf{k}^{-}\right)=\left[\frac{\left[C_{h, 0}\left(\mathbf{k}^{-}\right)-1\right] D_{+}\left(\mathbf{k}^{-}\right) \hat{g}_{+}^{(0)}\left(\mathbf{k}^{+}\right)-u_{0}\left(\mathbf{k}^{+}\right)}{D_{+}\left(\mathbf{k}^{+}-\mathbf{k}^{-}\right)}\left[G^{(2)}\left(\mathbf{k}^{+}\right)-G^{(2)}(0)\right]\right.
$$

As shown in [BM2], by using the symmetry property

$$
\hat{g}_{\omega}^{(j)}(\mathbf{k})=-i \omega \hat{g}_{\omega}^{(j)}\left(\mathbf{k}^{*}\right) \quad, \quad \mathbf{k}=\left(k, k_{0}\right), \quad \mathbf{k}^{*}=\left(-k_{0}, k\right),
$$

$F_{2,+, \tilde{\omega}}^{(-1)}$ can be written as

$$
F_{2,+, \tilde{\omega}}^{-1}\left(\mathbf{k}^{+}, \mathbf{k}^{-}\right)=\frac{1}{D_{+}(\mathbf{p})}\left[p_{0} A_{0,+, \tilde{\omega}}\left(\mathbf{k}^{+}, \mathbf{k}^{-}\right)+p_{1} A_{1,+, \tilde{\omega}}\left(\mathbf{k}^{+}, \mathbf{k}^{-}\right)\right]
$$

where $A_{i,+, \tilde{\omega}}\left(\mathbf{k}^{+}, \mathbf{k}^{-}\right)$are functions such that, if we define

$$
\mathcal{L} F_{2,+, \tilde{\omega}}^{-1}=\frac{1}{D_{+}(\mathbf{p})}\left[p_{0} A_{0,+, \tilde{\omega}}(0,0)+p_{1} A_{1,+, \tilde{\omega}}(0,0)\right],
$$

then

$$
\mathcal{L} F_{2,+,+}^{-1}=Z_{-1}^{3,+} \quad, \quad \mathcal{L} F_{2,+,-}^{-1}=\frac{D_{-}(\mathbf{p})}{D_{+}(\mathbf{p})} Z_{-1}^{3,-},
$$

where $Z_{-1}^{3,+}$ and $Z_{-1}^{3,-}$ are suitable real constants. Hence the local part of the marginal term (4.32) is, by definition, equal to

$$
Z_{-1}^{3,+} T_{+}\left(\psi^{[h,-1]}\right)+Z_{-1}^{3,-} T_{-}\left(\psi^{[h,-1]}\right)
$$

Let us finally consider the terms contributing to $\overline{\mathcal{V}}_{b, 2}^{(-1)}$, that is those where $\hat{\psi}_{\mathbf{\mathbf { k }}_{4}-\mathbf{p}}^{+}$is not contracted and there is a vertex of type $T_{+}$or $T_{-}$. If even this vertex is not contracted, we get a contribution similar to (4.39), with $\nu_{ \pm}$in place of $Z_{-1}^{3, \pm}$. Among the terms with at least one vertex $\lambda$, there is, as before, no term with two external lines; hence the only marginal terms have four external lines and can be written in the form

$$
\begin{aligned}
& \int d \mathbf{p} \tilde{\chi}_{M}(\mathbf{p}) \hat{J}_{\mathbf{k}_{4}} \hat{g}_{-}\left(\mathbf{k}_{4}\right) \int d \mathbf{k}^{+} \sum_{\tilde{\omega}} \hat{\psi}_{\mathbf{k}^{+}, \tilde{\omega}}^{+} \hat{\psi}_{\mathbf{k}^{+}-\mathbf{p}, \tilde{\omega}}^{-} \cdot \\
& \cdot\left[\nu_{+} G_{+, \tilde{\omega}}^{(0)}\left(\mathbf{k}^{+}, \mathbf{k}^{+}-\mathbf{p}\right)+\nu_{-} \frac{D_{-}(\mathbf{p})}{D_{+}(\mathbf{p})} G_{-, \tilde{\omega}}^{(0)}\left(\mathbf{k}^{+}, \mathbf{k}^{+}-\mathbf{p}\right)\right] .
\end{aligned}
$$

By using the symmetry property (4.35) of the propagators, it is easy to show that $G_{\omega,-\omega}^{(0)}(0,0)=0$. Hence, if we regularize (4.40) by subtracting $G_{\omega, \tilde{\omega}}^{(0)}(0,0)$ to $G_{\omega, \tilde{\omega}}^{(0)}\left(\mathbf{k}^{+}, \mathbf{k}^{+}-\mathbf{p}\right)$, we still get a local term of the form (4.39). 
By collecting all the local term, we can write

$$
\mathcal{L}\left[\overline{\mathcal{V}}_{b, 1}^{(-1)}+\overline{\mathcal{V}}_{b, 2}^{(-1)}\right]\left(\psi^{[h,-1]}\right)=\nu_{-1,+} T_{+}\left(\psi^{[h,-1]}\right)+\nu_{-1,-} T_{-}\left(\psi^{[h,-1]}\right)
$$

where $\nu_{-1, \omega}=\nu_{\omega}+Z_{-1}^{3, \omega}+G_{\omega, \omega}^{(0)}(0,0)$. Hence

$$
\begin{aligned}
& \overline{\mathcal{V}}^{(-1)}\left(\psi^{[h,-1]}\right)=T_{1}\left(\psi^{[h,-1]}\right)+\nu_{-1,+} T_{+}\left(\psi^{[h,-1]}\right)+\nu_{-1,-} T_{-}\left(\psi^{[h,-1]}\right)+ \\
& +\left[\tilde{\lambda}_{-1} \bar{F}_{\lambda}^{[h,-1]}\left(\psi^{[h,-1]}\right)+\tilde{z}_{-1} \hat{\psi}_{\overline{\mathbf{k}}_{4},-}^{[h,-1]+} D_{-}\left(\overline{\mathbf{k}}_{4}\right)\right] \hat{g}_{-}\left(\overline{\mathbf{k}}_{4}\right) \hat{J}_{\overline{\mathbf{k}}_{4}}+\overline{\mathcal{V}}_{R}^{(-1)}\left(\psi^{[h,-1]}\right),
\end{aligned}
$$

where $\overline{\mathcal{V}}_{R}^{(-1)}\left(\psi^{[h,-1]}\right)$ is the sum of all irrelevant terms linear in the external field $J$.

4.4 The multiscale expansion of $\tilde{G}_{+}^{4}\left(\overline{\mathbf{k}}_{1}, \overline{\mathbf{k}}_{2}, \overline{\mathbf{k}}_{3}, \overline{\mathbf{k}}_{4}\right)$ : the higher scales integration.

The integration of the field $\psi^{(-1)}$ is done in a similar way; we shall call $\overline{\mathcal{V}}^{(-2)}\left(\psi^{[h,-2]}\right)$ the sum over all terms linear in $J$. As before, the condition (3.28) on the external momenta $\mathbf{k}_{i}$ forbids the presence of vertices of type $\varphi$, if $h<-1$, as we shall suppose.

The main difference is that there is no contribution obtained by contracting both field variables belonging to $\delta \rho$ in $T_{1}(\psi)$ at scale -1 , because of (4.13). It is instead possible to get marginal terms with four external lines (two is impossible), such that one of these fields is contracted at scale -1 . However, in this case, the second field variable will be necessarily contracted at scale $h$, so that we can put $\mathcal{R}=1$ for such terms; in fact, the extra factor $\gamma^{-(-1-h)}$ coming from the bound (4.22) after the integration of the last scale field, has the effect of automatically regularize them, and even the terms containing them as subgraphs.

The terms with a $T_{1}$ vertex, such that the field variables belonging to $\delta \rho$ are not contracted, can be treated as in $\S 4.3$, hence do not need a regularization.

It follows that, if the irrelevant part $\overline{\mathcal{V}}_{R}^{(-1)}$ were absent in the r.h.s. of (4.42), then the regularization procedure would not produce any local term proportional to $\bar{F}_{\lambda}^{[h,-1]}\left(\psi^{[h,-2]}\right)$, starting from a graph containing a $T_{1}$ vertex.

It is easy to see that all other terms containing a vertex of type $T_{1}$ or $T_{ \pm}$can be treated as in $\S 4.3$. Moreover, the support properties of $\hat{g}_{-}\left(\overline{\mathbf{k}}_{4}\right)$ immediately implies that it is not possible to produce a graph contributing to $\overline{\mathcal{V}}^{(-2)}$, containing the $\tilde{z}_{-1}$ vertex. Hence, in order to complete the analysis of $\overline{\mathcal{V}}^{(-2)}$, we still have to consider the marginal terms containing the $\tilde{\lambda}_{-1}$ vertex, for which we simply apply the localization procedure defined in (4.26), (4.27). We shall define two new constants $\tilde{\lambda}_{-2}$ and $\tilde{z}_{-2}$, so that $\tilde{\lambda}_{-2}\left(Z_{-2}\right)^{2}$ is the coefficient of the local term proportional to $\bar{F}_{\lambda}^{[h,-1]}\left(\psi^{[h,-2]}\right)$, while $\tilde{z}_{-2} Z_{-2} \hat{\psi}_{\overline{\mathbf{k}}_{4},-}^{[h,-2]+} D_{-}\left(\overline{\mathbf{k}}_{4}\right) \hat{g}_{-}\left(\overline{\mathbf{k}}_{4}\right) \hat{J}_{\mathbf{k}_{4}}$ denotes the sum of all local terms with two external lines produced in the second integration step.

The above procedure can be iterated up to scale $h+1$, without any important difference. In particular, for all marginal terms (necessarily with four external lines) such that one of the field variables belonging to $\delta \rho$ in $T_{1}(\psi)$ is contracted at scale $i \geq j$, we put $\mathcal{R}=1$. We can do that, because, in this case, the second field variable belonging to $\delta \rho$ has to be contracted at scale $h$, so that the extra factor $\gamma^{-(i-h)}$ of (4.22) has the effect of automatically regularize their contribution to the tree expansion of $\tilde{G}_{+}^{4}\left(\overline{\mathbf{k}}_{1}, \overline{\mathbf{k}}_{2}, \overline{\mathbf{k}}_{3}, \overline{\mathbf{k}}_{4}\right)$, to be described later.

Note that, as in the case $j=-1$, there is no problem connected with the presence of the factors $\tilde{\chi}(\mathbf{p})$ and $D_{-}(\mathbf{p}) D_{+}(\mathbf{p})^{-1}$. In fact, if the field $\hat{\psi}_{\mathbf{k}_{4}-\mathbf{p},-}^{+}$appearing in the definition of $T_{1}(\psi)$ or $T_{ \pm}(\psi)$ is contracted on scale $j$, each momentum derivative related with the regularization procedure produces the right $\gamma^{-j}$ dimensional factor, since $\mathbf{p}$ is of order $\gamma^{j}$ and the derivatives of $\tilde{\chi}(\mathbf{p})$ are different from 0 only for momenta of order $\gamma^{h}$. If, on the contrary, the field $\hat{\psi}_{\mathbf{k}_{4}-\mathbf{p},-}^{+}$is not contracted, then the renormalization procedure is tuned so that $\tilde{\chi}(\mathbf{p})$ and $D_{-}(\mathbf{p}) D_{+}(\mathbf{p})^{-1}$ are not affected by the regularization procedure. 
At step $-j$, we get an expression of the form

$$
\begin{aligned}
& \overline{\mathcal{V}}^{(j)}\left(\psi^{[h, j]}\right)=T_{1}\left(\psi^{[h, j]}\right)+\nu_{j,+} T_{+}\left(\psi^{[h, j]}\right)+\nu_{j,-} T_{-}\left(\psi^{[h, j]}\right)+ \\
& +\left[\tilde{\lambda}_{j} Z_{j}^{2} \bar{F}_{\lambda}^{[h, j]}\left(\psi^{[h, j]}\right)+\sum_{i=j}^{-1} \tilde{z}_{i} Z_{i} \hat{\psi}_{\overline{\mathbf{k}}_{4},-}^{[h, j]+} D_{-}\left(\overline{\mathbf{k}}_{4}\right)\right] \hat{g}_{-}\left(\overline{\mathbf{k}}_{4}\right) \hat{J}_{\overline{\mathbf{k}}_{4}}+\overline{\mathcal{V}}_{R}^{j}\left(\psi^{[h,-1]}\right),
\end{aligned}
$$

where $\overline{\mathcal{V}}_{R}^{j}\left(\psi^{[h,-1]}\right)$ is thought as a convergent tree expansion (under the hypothesis that $\bar{\lambda}_{h}$ is small enough), to be described in $\S 4.5$. Since $Z_{-1}=1$, this expression is in agreement with (4.42).

The expansion of $\tilde{G}_{+}^{4}\left(\overline{\mathbf{k}}_{1}, \overline{\mathbf{k}}_{2}, \overline{\mathbf{k}}_{3}, \overline{\mathbf{k}}_{4}\right)$ is obtained by building all possible graphs with four external lines, which contain one term taken from the expansion of $\overline{\mathcal{V}}^{(h)}\left(\psi^{(h)}\right)$ and an arbitrary number of terms taken from the effective potential $\mathcal{V}^{(h)}\left(\psi^{(h)}\right)$. One of the external lines is associated with the free propagator $g_{-}\left(\overline{\mathbf{k}}_{4}\right)$, the other three are associated with propagators of scale $h$ and momenta $\overline{\mathbf{k}}_{i}, i=1,2,3$.

Remark. With respect to the expansion for $G_{+}^{4}$, there are three additional quartic running coupling constants, $\nu_{j,+}, \nu_{j,-}$ and $\tilde{\lambda}_{j}$. Note that they are all $O(\lambda)$, despite of the fact that the interaction $T_{1}$ has a coupling $O(1)$; this is a crucial property, which follows from the properties in $\S 4.2$, implying that either $T_{1}$ is contracted at scale 0 , or it gives no contribution to the running coupling constants. At a first sight, it seems that now we have a problem more difficult than the initial one; we started from the expansion for $G_{+}^{4}$, which is convergent if the running coupling $\lambda_{j}$ is small, and we have reduced the problem to that of controlling the flow of four running coupling constants, $\nu_{+, j}, \nu_{j,-}$, $\lambda_{j}, \tilde{\lambda}_{j}$. However, we will see that, under the hypothesis $\bar{\lambda}_{h} \leq \varepsilon_{1}$, also the flow of $\nu_{j,+}, \nu_{j,-}, \tilde{\lambda}_{j}$ is bounded; one uses the counterterms $\nu_{+}, \nu_{-}$(this is the reason why we introduced them in $\S 3$ ) to impose that $\nu_{+, j}, \nu_{j,-}$ are decreasing and vanishing at $j=h$, and then that the beta functions for $\tilde{\lambda}_{j}$ and $\lambda_{j}$ are identical up to exponentially decaying $O\left(\gamma^{\tau j}\right)$ terms.

4.5 The tree structure of the expansion.

In order to describe the tree expansion of $\overline{\mathcal{V}}^{(j)}\left(\psi^{[h, j]}\right), j \in[h+1,-1]$, and $\tilde{G}_{+}^{4}\left(\overline{\mathbf{k}}_{1}, \overline{\mathbf{k}}_{2}, \overline{\mathbf{k}}_{3}, \overline{\mathbf{k}}_{4}\right)$, we have to modify the tree definition given in $\S 2$, after Fig. 11, in the following way.

1) Instead of two, there are six types of special endpoints. There are still the endpoints of type $\varphi$, defined exactly as before, but there is no endpoint of type $J$. In addition, we have special endpoints of type $T_{1}, T_{+}, T_{-}, \tilde{\lambda}$ and $\tilde{z}$, associated in an obvious way with the five local terms of (4.43).

2) There are only trees with one and only one special endpoint of type different from $\varphi$.

3) The scale index is $\leq+1$ for the endpoints of type $T_{1}, T_{+}$or $T_{-}$, while it is $\leq 0$ for the endpoints of type $\tilde{\lambda}$ or $\tilde{z}$. Moreover, the scale index of an endpoint $v$ of type $T_{1}, T_{+}, T_{-}$or $\tilde{\lambda}$ is equal to $h_{v^{\prime}}+1$, if $v^{\prime}$ is the non trivial vertex immediately preceding $v$.

4 ) If the tree has more than one endpoint and one of them is of type $\tilde{z}$, the vertex $v_{0}$ of the tree must have scale $h$ and its scale index is equal to any value between $h+1$ and 0 .

5 ) Given a tree with one endpoint $v_{1}$ of type $T_{1}$ and scale index $h_{v_{1}}=+1$, the $\mathcal{R}$ operation in the non trivial vertices of the path $\mathcal{C}$ connecting $v_{1}$ to $v_{0}$ depends on the set $P_{1}$ of external lines in the vertex $v_{1}^{\prime}$ (of scale 0 ) immediately preceding $v_{1}$.

If $P_{1}$ contains no one of the two external lines of $v_{1}$ belonging to the $\delta \rho$ part of the corresponding $T_{1}(\psi)$ term, then $\mathcal{R}$ is defined in agreement with the localization procedure bringing to (4.38) for all vertices $v \in \mathcal{C}$, such that $v>v_{2}, v_{2}$ being the higher vertex, possibly coinciding with $v_{1}^{\prime}$, whose set of external lines does not contain the field $\hat{\psi}_{\mathbf{k}_{4}-\mathbf{p}}^{+}$of $T_{1}(\psi)$. For the remaining vertices of $\mathcal{C}, \mathcal{R}$ is defined in the usual way.

If $P_{1}$ contains both the two external lines of $v_{1}$ belonging to $\delta \rho$ (hence the line of momentum $\overline{\mathbf{k}}_{4}-\mathbf{p}$ can not belong to $P_{1}$ ), we define $\mathcal{R}$ in agreement with the remark following (4.24), up to the higher vertex $v_{2}<v_{1}$, where at least one of the lines of $\delta \rho$ does not belong to $P_{1}$ anymore. For $v \leq v_{2}, \mathcal{R}$ is defined in the usual way.

$11 /$ ottobre $/ 2018 ; 1: 16$ 
If $P_{1}$ contains only one of the two external lines of $v_{1}$ belonging to $\delta \rho$ and one defines $v_{2}$ as before, $\mathcal{R}$ is defined along $\mathcal{C}$ in agreement with the obvious generalization of (4.34), for $v>v_{2}$. In $v_{2}$ one has to introduce a new label to distinguish two cases, related with the two different terms in the braces of the r.h.s. of (4.28). In the first case, $\mathcal{R}=1$ for all $v \leq v_{2}$, in the second case $\mathcal{R}$ is defined in the usual way.

If $h_{v_{1}} \leq 0$ and we define $P_{1}$ and $v_{2}$ as before, the set $P_{1}$, as well as the set $P_{v}$ for all $v<v_{1}$, must contain at least one of the two external lines of $v_{1}$ belonging to $\delta \rho$. Moreover, if $P_{1}$ contains one of these lines, then $\mathcal{R}=1$ for all $v \leq v_{2}$.

6) A similar, but simpler, discussion can be done for the trees containing an endpoint of type $T_{ \pm}$. We do not give the details, but only stress that there is now no vertex $v$ with $\left|P_{v}\right|=2$ or $\left|P_{v}\right|=4$, for which $\mathcal{R}=1$.

\subsection{The flow of $\nu_{j, \pm}$.}

The definitions of the previous sections imply that there is no contribution to $\nu_{j, \pm}$, coming from trees with a special endpoint of type $\tilde{\lambda}$ or $\tilde{z}$. Moreover, because of the symmetry (4.35) of the propagators (see remark after (4.40)), $\nu_{j,+}$ gets no contribution from trees with a special endpoint of type $\nu_{j,-}$, and viceversa. Finally, and very important, if a tree contributing to $\nu_{j, \pm}$ has an endpoint of type $T_{1}$, this endpoint must have scale index +1 .

The following Lemma has an important role in the following.

LEMma 4.1 If $\bar{\lambda}_{h}$ is small enough ( uniformly in $h$ ), it is possible to choose $\nu_{+}$and $\nu_{-}$so that $\nu_{h, \omega}=0$ and

$$
\left|\nu_{j, \omega}\right| \leq c_{0} \bar{\lambda}_{h} \gamma^{\theta j} \quad, \quad h+1 \leq j \leq 0,
$$

where $0<\theta<1 / 4, c_{0}$ is a suitable constant, independent of $h$, and $\nu_{0, \omega}=\nu_{\omega}$.

Proof - The previous remarks imply that there exists $\bar{\varepsilon}_{1} \leq \bar{\varepsilon}_{0}$, such that, if $\bar{\lambda}_{h} \leq \bar{\varepsilon}_{1}$, we can write

$$
\nu_{j-1, \omega}=\nu_{j, \omega}+\beta_{\nu, \omega}^{(j)}\left(\lambda_{j}, \nu_{j, \omega} ; \ldots ; \lambda_{0}, \nu_{0, \omega}\right) \quad, \quad h+1 \leq j \leq 0,
$$

with $\lambda_{0}=\lambda, \nu_{0, \omega}=\nu_{\omega}$ and

$$
\beta_{\nu, \omega}^{(j)}\left(\lambda_{j}, \nu_{j, \omega} ; \ldots ; \lambda_{0}, \nu_{0, \omega}\right)=\beta_{\nu, \omega}^{(j, 1)}\left(\lambda_{j} ; \ldots ; \lambda_{0}\right)+\sum_{j^{\prime}=j}^{0} \nu_{j^{\prime}, \omega} \tilde{\beta}_{\nu, \omega}^{\left(j, j^{\prime}\right)}\left(\lambda_{j} ; \ldots ; \lambda_{0}\right) .
$$

Moreover given a positive $\theta<1 / 4$, there are constants $c_{1}$ and $c_{2}$ such that

$$
\left|\beta_{\nu, \omega}^{(j, 1)}\left(\lambda_{j} ; \ldots ; \lambda_{0}\right)\right| \leq c_{1} \bar{\lambda}_{h} \gamma^{2 \theta j} \quad, \quad\left|\tilde{\beta}_{\nu, \omega}^{\left(j, j^{\prime}\right)}\left(\lambda_{j} ; \ldots ; \lambda_{0}\right)\right| \leq c_{2} \bar{\lambda}_{h}^{2} \gamma^{2 \theta\left(j-j^{\prime}\right)} .
$$

This follows from the fact that $\beta_{\nu, \omega}^{(j, 1)}$ and $\tilde{\beta}_{\nu, \omega}^{\left(j, j^{\prime}\right)}$ are given by a sum of trees verifying the bound (2.32) with $d_{v}>0$, with at least an end-point respectively at scale 0 and at scale $j^{\prime}$, hence one can improve the bound respectively by a factor $\gamma^{2 \theta j}$ and $\gamma^{2 \theta\left(j-j^{\prime}\right)}$. In the following we shall call this property the short memory property. Note that the bound of $\tilde{\beta}_{\nu, \omega}^{\left(j, j^{\prime}\right)}$ is of order $\bar{\lambda}_{h}^{2}$, instead of $\bar{\lambda}_{h}$, because of the symmetry (4.35), but a bound of order $\bar{\lambda}_{h}$ would be sufficient.

By a simple iteration, (4.45) can also be written in the form

$$
\nu_{j-1, \omega}=\nu_{0, \omega}+\sum_{j^{\prime}=j}^{0} \beta_{\nu, \omega}^{\left(j^{\prime}\right)}\left(\lambda_{j^{\prime}}, \nu_{j^{\prime}, \omega} ; \ldots ; \lambda_{0}, \nu_{0, \omega}\right) .
$$

We want to show that it is possible to choose $\nu_{0, \omega}$, so that $\nu_{0, \omega}$ is of order $\bar{\lambda}_{h}$ and $\nu_{h, \omega}=0$. Since this last condition, by (4.48), is equivalent to

$$
\nu_{0, \omega}=-\sum_{j=h+1}^{0} \beta_{\nu, \omega}^{(j)}\left(\lambda_{j}, \nu_{j, \omega} ; \ldots ; \lambda_{0}, \nu_{0, \omega}\right)
$$


we see, by inserting (4.49) in the r.h.s. of (4.48), that we have to show that there is a sequence $\underline{\nu}=\left\{\nu_{j}, h+1 \leq j \leq 0\right\}$, such that $\nu_{0, \omega}$ is of order $\bar{\lambda}_{h}$ and

$$
\nu_{j}=-\sum_{j^{\prime}=h+1}^{j} \beta_{\nu, \omega}^{\left(j^{\prime}\right)}\left(\lambda_{j^{\prime}}, \nu_{j^{\prime}}, . ., \lambda_{0}, \nu_{0}\right)
$$

In order to prove that, we introduce the space $\mathfrak{M}_{\theta}$ of the sequences $\underline{\nu}=\left\{\nu_{j}, h+1 \leq j \leq 0\right\}$ such that $\left|\nu_{j}\right| \leq c \bar{\lambda}_{h} \gamma^{\theta j}$, for some $c$; we shall think $\mathfrak{M}_{\theta}$ as a Banach space with norm $\|\underline{\nu}\|_{\theta}=$ $\sup _{h+1 \leq j \leq 0}\left|\nu_{j}\right| \gamma^{-\theta j} \bar{\lambda}_{h}^{-1}$. We then look for a fixed point of the operator $\mathbf{T}: \mathfrak{M}_{\theta} \rightarrow \mathfrak{M}_{\theta}$ defined as:

$$
(\mathbf{T} \underline{\nu})_{j}=-\sum_{j^{\prime}=h+1}^{j} \beta_{\nu, \omega}^{\left(j^{\prime}\right)}\left(\lambda_{j^{\prime}}, \nu_{j^{\prime}}, . ., \lambda_{0}, \nu_{0}\right)
$$

Note that, if $\bar{\lambda}_{h}$ is sufficiently small, then $\mathbf{T}$ leaves invariant the ball $\mathfrak{B}_{\theta}$ of radius $c_{0}=2 c_{1}$ $\sum_{n=0}^{\infty} \gamma^{-n}$ of $\mathfrak{M}_{\theta}, c_{1}$ being the constant in (4.47). In fact, by (4.46) and (4.47), if $\|\underline{\nu}\|_{\theta} \leq c_{0}$, then

$$
\left|(\mathbf{T} \underline{\nu})_{j}\right| \leq \sum_{j^{\prime}=h+1}^{j} c_{1} \bar{\lambda}_{h} \gamma^{2 \theta j^{\prime}}+\sum_{j^{\prime}=h+1}^{j} \sum_{i=j^{\prime}}^{0} c_{0} \bar{\lambda}_{h} \gamma^{\theta i} c_{2} \bar{\lambda}_{h}^{2} \gamma^{2 \theta\left(j^{\prime}-i\right)} \leq c_{0} \bar{\lambda}_{h} \gamma^{\theta j}
$$

if $2 c_{2} \bar{\lambda}_{h}^{2}\left(\sum_{n=0}^{\infty} \gamma^{-n}\right)^{2} \leq 1$.

$\mathbf{T}$ is a also a contraction on $\mathfrak{B}_{\theta}$, if $\bar{\lambda}_{h}$ is sufficiently small; in fact, if $\underline{\nu}, \underline{\nu^{\prime}} \in \mathfrak{M}_{\theta}$,

$$
\begin{aligned}
& \left|(\mathbf{T} \nu)_{j}-\left(\mathbf{T} \underline{\nu}^{\prime}\right)_{j}\right| \leq \sum_{j^{\prime}=h+1}^{j}\left|\beta_{\nu, \omega}^{\left(j^{\prime}\right)}\left(\lambda_{j^{\prime}}, \nu_{j^{\prime}}, . ., \lambda_{0}, \nu_{0}\right)-\beta_{\nu, \omega}^{\left(j^{\prime}\right)}\left(\lambda_{j^{\prime}}, \nu_{j^{\prime}}^{\prime}, ., \lambda_{0}, \nu_{0}^{\prime}\right)\right| \\
& \leq \sum_{j^{\prime}=h+1}^{j} \sum_{i=j^{\prime}}^{0}\left\|\underline{\nu}-\underline{\nu}^{\prime}\right\|_{\theta} \bar{\lambda}_{h} \gamma^{\theta i} c_{2} \bar{\lambda}_{h}^{2} \gamma^{2 \theta\left(j^{\prime}-i\right)} \leq \frac{1}{2}\left\|\underline{\nu}-\underline{\nu}^{\prime}\right\|_{\theta} \bar{\lambda}_{h} \gamma^{\theta j},
\end{aligned}
$$

if $c_{2} \bar{\lambda}_{h}^{2}\left(\sum_{n=0}^{\infty} \gamma^{-n}\right)^{2} \leq 1 / 2$. Hence, by the contraction principle, there is a unique fixed point $\underline{\nu}^{*}$ of $\mathbf{T}$ on $\mathfrak{B}_{\theta}$.

4.7 The constants $\tilde{\lambda}_{j}$ and $\tilde{z}_{j}$.

We shall now analyze the constants $\tilde{\lambda}_{j}$ and $\tilde{z}_{j}, h \leq j \leq-1$, appearing in the expansion of $\tilde{G}_{+}^{4}\left(\overline{\mathbf{k}}_{1}, \overline{\mathbf{k}}_{2}, \overline{\mathbf{k}}_{3}, \overline{\mathbf{k}}_{4}\right)$. We shall do that by comparing their values with the values of $\lambda_{j}$ and $z_{j}$. We start noting that the beta function equation for $\lambda_{j}$ can be written as

$$
\lambda_{j-1}=\left(\frac{Z_{j}}{Z_{j-1}}\right)^{2} \lambda_{j}+\beta_{j}+\beta_{j}^{(1)},
$$

where $\beta_{j}$ is the sum over the local parts of the trees with at least two endpoints and no endpoint of scale index +1 , while $\beta_{j}^{(1)}$ is the similar sum over the trees with at least one endpoint of scale index +1 .

On the other hand we can write

$$
\tilde{\lambda}_{j-1}=\left(\frac{Z_{j}}{Z_{j-1}}\right)^{2} \tilde{\lambda}_{j}+\tilde{\beta}_{j}+\tilde{\beta}_{j}^{(1)}+\tilde{\beta}_{j}^{(T)}+\tilde{\beta}_{j}^{(\nu)}
$$

where

1) $\tilde{\beta}_{j}$ is the sum over the local parts of the trees with at least two endpoints, no endpoint of scale index +1 and one special endpoint of type $\tilde{\lambda}$. 
2) $\tilde{\beta}_{j}^{(1)}+\tilde{\beta}_{j}^{(T)}$ is the sum over the trees with at least one endpoint of scale index +1 ; in this case, the special endpoint can be of type $\tilde{\lambda}$ or $T_{1}$ and, if it is of type $T_{1}$, its scale index must be equal to $+1 . \tilde{\beta}_{j}^{(1)}$ and $\tilde{\beta}_{j}^{(T)}$ are, respectively, the sum over the trees with the endpoint of type $\tilde{\lambda}$ or $T_{1}$.

3) $\tilde{\beta}_{j}^{(\nu)}$ is the sum over the trees with at least two endpoints, whose special endpoint is of type $T_{ \pm}$.

A crucial role in this paper has the following Lemma.

LEMmA 4.2 Let $\alpha=\tilde{\lambda}_{h} / \lambda_{h}$; then if $\bar{\lambda}_{h}$ is small enough, there exists a constant $c$, independent of $\lambda$, such that $|\alpha| \leq c$ and

$$
\left|\tilde{\lambda}_{j}-\alpha \lambda_{j}\right| \leq c \bar{\lambda}_{h} \gamma^{\theta j} \quad, \quad h+1 \leq j \leq-1
$$

Proof - The main point is the remark that there is a one to one correspondence between the trees contributing to $\beta_{j}$ and the trees contributing to $\tilde{\beta}_{j}$. In fact the trees contributing to $\tilde{\beta}_{j}$ have only endpoints of type $\lambda$, besides the special endpoint $v^{*}$, and the external field with $\omega=-$ and $\sigma=-$ has to belong to $P_{v^{*}}$. It follows that we can associate uniquely with any tree contributing to $\tilde{\beta}_{j}$ a tree contributing to $\beta_{j}$, by simply substituting the special endpoint with a normal endpoint, without changing any label. This correspondence is surjective, since we have imposed the condition that the trees contributing to $\tilde{\beta}_{j}$ and $\beta_{j}$ do not have endpoints of scale index +1 . Hence, we can write

$$
\left[\left(\frac{Z_{j}}{Z_{j-1}}\right)^{2}-1\right]\left(\tilde{\lambda}_{j}-\alpha \lambda_{j}\right)+\tilde{\beta}_{j}-\alpha \beta_{j}=\sum_{i=j}^{-1} \beta_{j, i}\left(\tilde{\lambda}_{i}-\alpha \lambda_{i}\right),
$$

where, thanks to the "short memory property" and the fact that $Z_{j} / Z_{j-1}=1+O\left(\bar{\lambda}_{j}^{2}\right)$, the constants $\beta_{j, i}$ satisfy the bound $\left|\beta_{j, i}\right| \leq C \bar{\lambda}_{j} \gamma^{2 \theta(j-i)}$, with $\theta$ defined as in Lemma 4.1.

Among the four last terms in the r.h.s. of (4.55), the only one depending on the $\tilde{\lambda}_{j}$ is $\tilde{\beta}_{j}^{(1)}$, which can be written in the form

$$
\tilde{\beta}_{j}^{(1)}=\sum_{i=j}^{-1} \beta_{j, i}^{\prime} \tilde{\lambda}_{i}
$$

the $\beta_{j, i}^{\prime}$ being constants which satisfy the bound $\left|\beta_{j, i}^{\prime}\right| \leq C \bar{\lambda}_{j} \gamma^{2 \theta j}$, since they are related to trees with an endpoint of scale index +1 . For the same reasons, we have the bounds $\left|\tilde{\beta}_{j}^{(T)}\right| \leq C \bar{\lambda}_{j} \gamma^{2 \theta j}$, $\left|\beta_{j}^{(1)}\right| \leq C \bar{\lambda}_{j}^{2} \gamma^{2 \theta j}$. Finally, by using also Lemma 4.1, we see that $\left|\tilde{\beta}_{j}^{(\nu)}\right| \leq C \bar{\lambda}_{j} \bar{\lambda}_{h} \gamma^{2 \theta j}$.

We now choose $\alpha$ so that

$$
\tilde{\lambda}_{h}-\alpha \lambda_{h}=0
$$

and we put

$$
x_{j}=\tilde{\lambda}_{j}-\alpha \lambda_{j} \quad, \quad h+1 \leq j \leq-1 .
$$

We can write

$$
x_{j-1}=x_{-1}+\sum_{j^{\prime}=j}^{-1}\left[\sum_{i=j^{\prime}}^{-1} \beta_{j^{\prime}, i} x_{i}+\sum_{i=j^{\prime}}^{-1} \beta_{j^{\prime}, i}^{\prime}\left(x_{i}+\alpha \lambda_{i}\right)+\tilde{\beta}_{j^{\prime}}^{(T)}+\tilde{\beta}_{j^{\prime}}^{(\nu)}-\alpha \beta_{j}^{(1)}\right] .
$$

On the other hand, the condition (4.59) implies that

$$
x_{-1}=-\sum_{j^{\prime}=h+1}^{-1}\left[\sum_{i=j^{\prime}}^{-1} \beta_{j^{\prime}, i} x_{i}+\sum_{i=j^{\prime}}^{-1} \beta_{j^{\prime}, i}^{\prime}\left(x_{i}+\alpha \lambda_{i}\right)+\tilde{\beta}_{j^{\prime}}^{(T)}+\tilde{\beta}_{j^{\prime}}^{(\nu)}-\alpha \beta_{j}^{(1)}\right],
$$


so that, if $h+1 \leq j \leq-1$, the $x_{j}$ satisfy the equation

$$
x_{j}=-\sum_{j^{\prime}=h+1}^{j}\left[\sum_{i=j^{\prime}}^{-1} \beta_{j^{\prime}, i} x_{i}+\sum_{i=j^{\prime}}^{-1} \beta_{j^{\prime}, i}^{\prime}\left(x_{i}+\alpha \lambda_{i}\right)+\tilde{\beta}_{j^{\prime}}^{(T)}+\tilde{\beta}_{j^{\prime}}^{(\nu)}-\alpha \beta_{j}^{(1)}\right] .
$$

We want to show that equation (4.63) has a unique solution satisfying the bound

$$
\left|x_{j}\right| \leq c_{0}\left(1+|\alpha| \bar{\lambda}_{h}\right) \bar{\lambda}_{h} \gamma^{\theta j},
$$

for a suitable constant $c_{0}$, independent of $h$, if $\bar{\lambda}_{h}$ is small enough. Hence we introduce the Banach space $\mathfrak{M}_{\theta}$ of sequences $\underline{x}=\left\{x_{j}, h+1 \leq j \leq-1\right\}$ with norm $\|\underline{x}\|_{\theta} \stackrel{\text { def }}{=} \sup _{j}\left|x_{j}\right| \gamma^{-\theta j} \bar{\lambda}_{h}^{-1}$ and look for a fixed point of the operator $\mathbf{T}: \mathfrak{M}_{\theta} \rightarrow \mathfrak{M}_{\theta}$ defined by the r.h.s. of (4.63). By using the bounds on the various constants appearing in the definition of $\mathbf{T}$, we can easily prove that there are two constants $c_{1}$ and $c_{2}$, such that

$$
\left|(\mathbf{T} \underline{x})_{j}\right| \leq c_{1} \bar{\lambda}_{h}\left(1+|\alpha| \bar{\lambda}_{h}\right) \gamma^{\theta j}+c_{2} \bar{\lambda}_{h} \sum_{j^{\prime}=h+1}^{j} \sum_{i=j^{\prime}}^{-1} \gamma^{2 \theta\left(j^{\prime}-i\right)}\left|x_{i}\right| .
$$

Hence, if we take $c_{0}=M c_{1}, M \geq 2$, the ball $\mathfrak{B}_{M}$ of radius $c_{0}\left(1+|\alpha| \bar{\lambda}_{h}\right)$ in $\mathfrak{M}_{\theta}$ is invariant under the action of $\mathbf{T}$, if $c_{2} \bar{\lambda}_{h}\left(\sum_{n=0}^{\infty} \gamma^{-n}\right)^{2} \leq 1 / 2$, since $1 / 2 \leq(M-1) / M$. On the other hand, under the same condition, $\mathbf{T}$ is a contraction in all $\mathfrak{M}_{\theta}$; in fact, if $\underline{x}, \underline{x}^{\prime} \in \mathfrak{M}_{\theta}$, then

$$
\left|(\mathbf{T} \underline{x})_{j}-\left(\mathbf{T} \underline{x}^{\prime}\right)_{j}\right| \leq c_{2} \bar{\lambda}_{h}^{2}\left\|\underline{x}-\underline{x}^{\prime}\right\| \sum_{j^{\prime}=h+1}^{j} \sum_{i=j^{\prime}}^{-1} \gamma^{2 \theta\left(j^{\prime}-i\right)} \gamma^{\theta i} \leq \frac{1}{2}\left\|\underline{x}-\underline{x}^{\prime}\right\| \bar{\lambda}_{h} \gamma^{\theta j},
$$

if $c_{2} \bar{\lambda}_{h}\left(\sum_{n=0}^{\infty} \gamma^{-n}\right)^{2} \leq 1 / 2$. It follows, by the contraction principle, that there is a unique fixed point in the ball $\mathfrak{B}_{M}$, for any $M \geq 2$, hence a unique fixed point in $\mathfrak{M}_{\theta}$, satisfying the condition (4.64) with $c_{0}=2 c_{1}$.

To complete the proof, we have to show that $\alpha$ can be bounded uniformly in $h$. In order to do that, we insert in the l.h.s. of (4.62) the definition of $x_{-1}$ and we bound the r.h.s. by using (4.64) and (4.65); we get

$$
\left|\tilde{\lambda}_{-1}-\alpha \lambda_{-1}\right| \leq c_{3} \bar{\lambda}_{h}+c_{4}|\alpha| \bar{\lambda}_{h}^{2},
$$

for some constants $c_{3}$ and $c_{4}$. Since $\left|\lambda_{-1}\right| \geq c_{5}|\lambda|, \tilde{\lambda}_{-1} \leq c_{6}|\lambda|$ and $\bar{\lambda}_{h} \leq 2|\lambda|$ by the inductive hypothesis, we have

$$
\left|\alpha \lambda_{-1}\right| \leq\left|\tilde{\lambda}_{-1}\right|+c_{3} \bar{\lambda}_{h}+c_{4}|\alpha| \bar{\lambda}_{h}^{2} \Rightarrow|\alpha| \leq\left(c_{6}+2 c_{3}+2 c_{4}|\alpha| \bar{\lambda}_{h}\right) / c_{5}
$$

so that, $|\alpha| \leq 2\left(c_{6}+2 c_{3}\right) / c_{5}$, if $4 c_{4} \bar{\lambda}_{h} \leq c_{5}$.

Remark. The above Lemma is based on the fact that $\lambda_{j}$ and $\tilde{\lambda}_{j}$ have the same Beta function, up to $O\left(\gamma^{\theta j}\right)$ terms (note that this is true thanks to our choice of the counterterms $\nu_{ \pm}$, which implies that $\nu_{j, \pm}$ are $\left.O\left(\gamma^{\theta j}\right)\right)$. Hence if $\lambda_{j}$ is small, the same is true for $\tilde{\lambda}_{j}$.

We want now to discuss the properties of the constants $\tilde{z}_{j}, h \leq j \leq-1$, by comparing them with the constants $z_{j}$, which are involved in the renormalization of the free measure, see (2.23). There is a tree expansion for the $z_{j}$, which can be written as

$$
z_{j}=\beta_{j}+\beta_{j}^{(1)}
$$


where $\beta_{j}$ is the sum over the trees without endpoints of scale index +1 , while $\beta_{j}^{(1)}$ is the sum of the others, satisfying the bound $\left|\beta_{j}^{(1)}\right| \leq C \bar{\lambda}_{h}^{2} \gamma^{\theta j}$. The tree expansion of the $\tilde{z}_{j}$ can be written as

$$
\tilde{z}_{j}=\tilde{\beta}_{j}+\tilde{\beta}_{j}^{(\nu)}+\tilde{\beta}_{j}^{(1)}
$$

where $\tilde{\beta}_{j}$ is the sum over the trees without endpoints of scale index +1 , such that the special endpoint is of type $\tilde{\lambda}, \tilde{\beta}_{j}^{(\nu)}$ is the sum over the trees whose special endpoint is of type $T_{ \pm}$, and $\tilde{\beta}_{j}^{(1)}$ is the sum over the trees with at least an endpoint of scale index +1 (in this case, if the special endpoint is of type $T_{1}$, its scale index must be +1 , see discussion in $\S 4.4$ ).

Since there is no tree contributing to $\tilde{\beta}_{j}^{(1)}$ without at least one $\lambda$ or $\tilde{\lambda}$ endpoint and since all trees contributing to it satisfy the "short memory property", by using Lemma 4.2 (which implies that $\left|\tilde{\lambda}_{j}\right| \leq C \bar{\lambda}_{h}$ ), we get the bound $\left|\tilde{\beta}_{j}^{(1)}\right| \leq C \bar{\lambda}_{h} \gamma^{\theta j}$. In a similar manner, by using Lemma 4.1, we see that $\left|\tilde{\beta}_{j}^{(\nu)}\right| \leq C \bar{\lambda}_{h}^{2} \gamma^{\theta j}$.

Let us now consider $\beta_{j}$ and $\tilde{\beta}_{j}$. By an argument similar to that used in the proof of Lemma 4.2 , we can write

$$
\tilde{\beta}_{j}-\alpha \beta_{j}=\sum_{i=j+1}^{-1} \beta_{j, i}\left(\tilde{\lambda}_{i}-\alpha \lambda_{i}\right),
$$

where $\alpha$ is defined as in Lemma 4.2 and $\left|\beta_{j, i}\right| \leq C \bar{\lambda}_{h} \gamma^{2 \theta j}$. Hence, Lemma 4.2 implies that

$$
\left|\tilde{z}_{j}-\alpha z_{j}\right| \leq C \bar{\lambda}_{h} \gamma^{\theta j}
$$

4.8 The bound of $\tilde{G}_{+}^{4}\left(\overline{\mathbf{k}}_{1}, \overline{\mathbf{k}}_{2}, \overline{\mathbf{k}}_{3}, \overline{\mathbf{k}}_{4}\right)$.

There are various classes of trees contributing to the tree expansion of $\tilde{G}_{+}^{4}\left(\overline{\mathbf{k}}_{1}, \overline{\mathbf{k}}_{2}, \overline{\mathbf{k}}_{3}, \overline{\mathbf{k}}_{4}\right)$, depending on the type of the special endpoint. Let us consider first the family $\mathcal{T}_{\tilde{\lambda}}$ of the trees with an endpoint of type $\tilde{\lambda}$. These trees have the same structure of those appearing in the expansion of $G_{+}^{4}\left(\overline{\mathbf{k}}_{1}, \overline{\mathbf{k}}_{2}, \overline{\mathbf{k}}_{3}, \overline{\mathbf{k}}_{4}\right)$, except for the fact that the external (renormalized) propagator of scale $h$ and momentum $\overline{\mathbf{k}}_{4}$ is substituted with the free propagator $\hat{g}_{-}\left(\overline{\mathbf{k}}_{4}\right)$. It follows, by using the bound $\left|\tilde{\lambda}_{j}\right| \leq C \bar{\lambda}_{h}$, that a tree with $n$ endpoint is bounded by $\left(C \bar{\lambda}_{h}\right)^{n} Z_{h}^{-1} \gamma^{-4 h}$, larger for a factor $Z_{h}$ with respect to what we need.

Let us now consider the family $\mathcal{T}_{\tilde{z}}$ of the trees with a special endpoint of type $\tilde{z}$. Given a tree $\tau \in \mathcal{T}_{\tilde{\lambda}}$, we can associate with it the class $\mathcal{T}_{\tilde{z}, \tau}$ of all $\tau^{\prime} \in \mathcal{T}_{\tilde{\lambda}}$, obtained by $\tau$ in the following way:

1) we substitute the endpoint $v^{*}$ of type $\tilde{\lambda}$ of $\tau$ with an endpoint of type $\lambda$;

2) we link the endpoint $v^{*}$ to an endpoint of type $\tilde{z}$ trough a renormalized propagator of scale $h$. Note that $\mathcal{T}_{\tilde{z}}=\cup_{\tau \in \mathcal{T}_{\tilde{\lambda}}} \mathcal{T}_{\tilde{z}, \tau}$ and that, if $\tau$ has $n$ endpoints, any $\tau^{\prime} \in \mathcal{T}_{\tilde{z}, \tau}$ has $n+1$ endpoints. Moreover, since the value of $\overline{\mathbf{k}}_{4}$ has be chosen so that $f_{h}\left(\overline{\mathbf{k}}_{4}\right)=1, \hat{g}_{-}^{(h)}\left(\overline{\mathbf{k}}_{4}\right)=Z_{h-1}^{-1} \hat{g}_{-}\left(\overline{\mathbf{k}}_{4}\right)$; hence it is easy to show that the sum of the values of a tree $\tau \in \mathcal{T}_{\tilde{\lambda}}$, such the special endpoint has scale index $j^{*}+1$, and of all $\tau^{\prime} \in \mathcal{T}_{\tilde{z}, \tau}$ is obtained from the value of $\tau$, by substituting $\tilde{\lambda}_{j^{*}}$ with

$$
\Lambda_{j^{*}}=\tilde{\lambda}_{j^{*}}-\lambda_{j^{*}} \frac{\sum_{j=h}^{-1} \tilde{z}_{j} Z_{j}}{Z_{h-1}}
$$

see Fig. 10.

On the other hand, (4.72) and the bound $Z_{j} \leq \gamma^{-C \bar{\lambda}_{h}^{2} j}$, see [BM1], imply that, if $\bar{\lambda}_{h}$ is small enough

$$
\sum_{j=h}^{-1}\left|\tilde{z}_{j} Z_{j}-\alpha z_{j} Z_{j}\right| \leq \sum_{j=h}^{-1} C \bar{\lambda}_{h} \gamma^{\theta j} Z_{j} \leq C \bar{\lambda}_{h} .
$$


It follows, by using also the bound (4.56), that

$$
\Lambda_{j^{*}}=\alpha \lambda_{j^{*}}\left[1-\frac{\sum_{j=h}^{-1} z_{j} Z_{j}}{Z_{h-1}}\right]+\frac{O\left(\bar{\lambda}_{h}\right)}{Z_{h}} .
$$

Moreover, since $Z_{j-1}=Z_{j}\left(1+z_{j}\right)$, for $j \in[-1, h]$, and $Z_{-1}=1$, it is easy to check that

$$
Z_{h-1}-\sum_{j=h}^{-1} z_{j} Z_{j}=1 .
$$

This identity, Lemma 4.2 and (4.75) imply the bound

$$
\left|\Lambda_{j^{*}}\right| \leq C \frac{\bar{\lambda}_{h}}{Z_{h}}
$$

which gives us the "missing" $Z_{h}^{-1}$ factor for the sum over the trees whose special endpoint is of type $\tilde{\lambda}$ or $\tilde{z}$.

Let us now consider the family $\mathcal{T}_{\nu}$ of the trees with a special endpoint of type $T_{ \pm}$. It is easy to see, by using Lemma 4.1 and the "short memory property", that the sum over the trees of this class with $n \geq 0$ normal endpoints is bounded, for $\bar{\lambda}_{h}$ small enough, by $\left(C \bar{\lambda}_{h}\right)^{n+1} Z_{h}^{-1} \gamma^{-4 h} \sum_{j=h}^{-1} Z_{j}^{-2} \gamma^{2 \theta(h-j)}$ $\gamma^{\theta j} \leq\left(C \bar{\lambda}_{h}\right)^{n+1} Z_{h}^{-3} \gamma^{-(4-\theta) h}$, which is even better of our needs.

We still have to consider the family $\mathcal{T}_{1}$ of the trees with a special endpoint of type $T_{1}$. There is first of all the trivial tree, obtained by contracting all the $\psi$ lines of $T_{1}$ on scale $h$, but its value is 0 , because of the support properties of the function $\tilde{\chi}(\mathbf{p})$. Let us now consider a tree $\tau \in \mathcal{T}_{1}$ with $n \geq 1$ endpoints of type $\lambda$, whose structure is described in item 5) of $\S 4.5$, which we shall refer to for notation. If we put $h_{v_{1}}=j_{1}+1$ and $h_{v_{2}}=j_{2}$, then the dimensional bound of this tree differs from that of a tree with $n+1$ normal endpoints contributing to $G_{+}^{4}\left(\overline{\mathbf{k}}_{1}, \overline{\mathbf{k}}_{2}, \overline{\mathbf{k}}_{3}, \overline{\mathbf{k}}_{4}\right)$ for the following reasons:

1) there is a factor $Z_{h}^{-1}$ missing, because the external (renormalized) propagator of scale $h$ and momentum $\overline{\mathbf{k}}_{4}$ is substituted with the free propagator $\hat{g}_{-}\left(\overline{\mathbf{k}}_{4}\right)$;

2) there is a factor $\left|\lambda_{j_{1}}\right| Z_{j_{1}}^{2}$ missing, because there is no external field renormalization in the $T_{1}\left(\psi^{[h, j]}\right)$ contribution to $\overline{\mathcal{V}}^{(j)}\left(\psi^{[h, j]}\right)$, see (4.43);

3 ) if $P_{1}$ contains only one of the two external lines of $v_{1}$ belonging to $\delta \rho$, then there is a factor $\gamma^{-\left(j_{2}-h\right)}$ missing, because the absence of regularization in the vertices $v \leq v_{2}$, but this is compensated by the same factor arising because of the bound (4.22), see discussion after (4.28) and in $\S 4.4$, so that the "short memory property" is always satisfied;

4) there is a factor $Z_{h}^{-1}$ missing, because of the remark following (4.22).

It follows that the sum of the values of all trees $\tau \in \mathcal{T}_{1}$ with $n \geq 1$ normal endpoints, if $\bar{\lambda}_{h}$ is small enough, is bounded by $\left(C \bar{\lambda}_{h}\right)^{n} \gamma^{-4 h} \sum_{j_{1}=h}^{0} Z_{j_{1}}^{-2} \gamma^{2 \theta\left(h-j_{1}\right)} \leq\left(C \bar{\lambda}_{h}\right)^{n} \gamma^{-4 h} Z_{h}^{-2}$.

By collecting all the previous bounds, we prove that the bound (3.34) of Lemma 3.1 is satisfied in the case of $H_{+}^{4,1}$.

Remark. In $T_{1}$ and in the Grassmannian monomials multiplying $\nu_{j,+}, \nu_{j,-}$, an external line is always associated to a free propagator $\hat{g}_{-}\left(\overline{\mathbf{k}}_{4}\right)$; this is due to the fact that, in deriving the Dyson equation (3.10), one extracts a free propagator. Then in the bounds there is a $Z_{h}$ missing (such propagator is not "dressed" in the multiscale integration procedure), and at the end the crucial identity (4.76) has to be used to "dress" the extracted propagator carrying momentum $\overline{\mathbf{k}}_{4}$.

4.9 The bound in the case of $H_{-}^{4,1}$.

If we substitute, in the l.h.s. of (4.1) $H_{+}^{4,1}$ with $H_{-}^{4,1}$, we can proceed in a similar way. By using (3.23), we get

$$
\hat{g}_{-}\left(\mathbf{k}_{4}\right) \frac{1}{L \beta} \sum_{\mathbf{p}} \tilde{\chi}_{M}(\mathbf{p}) D_{+}^{-1}(\mathbf{p}) \hat{H}_{-}^{4,1}\left(\mathbf{p} ; \mathbf{k}_{1}, \mathbf{k}_{2}, \mathbf{k}_{3}, \mathbf{k}_{4}-\mathbf{p}\right)=
$$




$$
\begin{aligned}
= & \hat{g}_{-}\left(\mathbf{k}_{4}\right) \frac{1}{L \beta} \sum_{\mathbf{p}} \tilde{\chi}_{M}(\mathbf{p}) \frac{1}{L \beta} \sum_{\mathbf{k}} \frac{C_{-}(\mathbf{k}, \mathbf{k}-\mathbf{p})}{D_{+}(\mathbf{p})}<\hat{\psi}_{\mathbf{k},-}^{+} \hat{\psi}_{\mathbf{k}-\mathbf{p},-}^{-} ; \hat{\psi}_{\mathbf{k}_{1},+}^{-} ; \hat{\psi}_{\mathbf{k}_{2},+}^{+} ; \hat{\psi}_{\mathbf{k}_{3},-}^{-} ; \hat{\psi}_{\mathbf{k}_{4}-\mathbf{p},-}^{+}>^{T}+ \\
& -\nu_{-}^{\prime} \hat{g}_{-}\left(\mathbf{k}_{4}\right) \frac{1}{L \beta} \sum_{\mathbf{p}} \tilde{\chi}_{M}(\mathbf{p}) \frac{1}{L \beta} \sum_{\mathbf{k}} \frac{D_{-}(\mathbf{p})}{D_{+}(\mathbf{p})}<\hat{\psi}_{\mathbf{k},-}^{+} \hat{\psi}_{\mathbf{k}-\mathbf{p},-}^{-} ; \hat{\psi}_{\mathbf{k}_{1},+}^{-} ; \hat{\psi}_{\mathbf{k}_{2},+}^{+} ; \hat{\psi}_{\mathbf{k}_{3},+}^{-} ; \hat{\psi}_{\mathbf{k}_{4}-\mathbf{p},-}^{+}>^{T}- \\
& -\nu_{+}^{\prime} \hat{g}_{-}\left(\mathbf{k}_{4}\right) \frac{1}{L \beta} \sum_{\mathbf{p}} \tilde{\chi}_{M}(\mathbf{p}) \frac{1}{L \beta} \sum_{\mathbf{k}}<\hat{\psi}_{\mathbf{k},+}^{+} \hat{\psi}_{\mathbf{k}-\mathbf{p},+}^{-} ; \hat{\psi}_{\mathbf{k}_{1},+}^{-} ; \hat{\psi}_{\mathbf{k}_{2},+}^{+} ; \hat{\psi}_{\mathbf{k}_{3},-}^{-} ; \hat{\psi}_{\mathbf{k}_{4}-\mathbf{p},-}^{+}>^{T}
\end{aligned}
$$

We define $\tilde{G}_{-}^{4}\left(\mathbf{k}_{1}, \mathbf{k}_{2}, \mathbf{k}_{3}, \mathbf{k}_{4}\right)$ as in (4.2) with $\tilde{W}$ replaced by $\tilde{W}_{-}$given by

$$
\begin{gathered}
\tilde{W}_{-}=\log \int P(d \hat{\psi}) e^{-T_{2}(\psi)+\nu_{+}^{\prime} T_{+}(\psi)+\nu_{-}^{\prime} T_{-}(\psi)} e^{-V(\hat{\psi})+\sum_{\omega} \int d \mathbf{x}\left[\phi_{\mathbf{x}, \omega}^{+} \hat{\psi}_{\mathbf{x}, \omega}^{-}+\hat{\psi}_{\mathbf{x}, \omega}^{+} \phi_{\mathbf{x}, \omega}^{-}\right]} \\
T_{2}(\psi)=\frac{1}{L \beta} \sum_{\mathbf{p}} \tilde{\chi}_{M}(\mathbf{p}) \frac{1}{L \beta} \sum_{\mathbf{k}} \frac{C_{-}(\mathbf{k}, \mathbf{k}-\mathbf{p})}{D_{+}(\mathbf{p})}\left(\hat{\psi}_{\mathbf{k},-}^{+} \hat{\psi}_{\mathbf{k}-\mathbf{p},-}^{-}\right) \hat{\psi}_{\mathbf{k}_{4}-\mathbf{p},-}^{+} \hat{J}_{\mathbf{k}_{4}} \hat{g}\left(\mathbf{k}_{4}\right)
\end{gathered}
$$

$T_{+}, T_{-}$being defined as in (4.5), (4.6). By the analogues of (4.9), (4.10) we obtain

$$
-\tilde{G}_{-}^{4}\left(\mathbf{k}_{1}, \mathbf{k}_{2}, \mathbf{k}_{3}, \mathbf{k}_{4}\right)=g_{-}\left(\mathbf{k}_{4}\right) \frac{1}{L \beta} \sum_{\mathbf{p}} \tilde{\chi}_{M}(\mathbf{p}) \frac{H_{-}^{4,1}\left(\mathbf{p} ; \mathbf{k}_{1}, \mathbf{k}_{2}, \mathbf{k}_{3}, \mathbf{k}_{4}-\mathbf{p}\right)}{D_{+}(\mathbf{p})} .
$$

The calculation of $\tilde{G}_{-}^{4}\left(\mathbf{k}_{1}, \mathbf{k}_{2}, \mathbf{k}_{3}, \mathbf{k}_{4}\right)$ is done via a multiscale expansion essentially identical to the one of $\tilde{G}_{+}^{4}\left(\mathbf{k}_{1}, \mathbf{k}_{2}, \mathbf{k}_{3}, \mathbf{k}_{4}\right)$, by taking into account that $\delta \rho_{\mathbf{p},+}$ has to be substituted with

$$
\delta \rho_{\mathbf{p},-}=\frac{1}{\beta L} \sum_{\mathbf{k}} \frac{C_{-}(\mathbf{p}, \mathbf{k})}{D_{+}(\mathbf{p})}\left(\hat{\psi}_{\mathbf{k},-}^{+} \hat{\psi}_{\mathbf{k}-\mathbf{p},-}^{-}\right) .
$$

Let us consider the first step of the iterative integration procedure and let us call again $\overline{\mathcal{V}}^{(-1)}\left(\psi^{[h,-1]}\right)$ the contribution to the effective potential of the terms linear in $J$. Let us now decompose $\overline{\mathcal{V}}^{(-1)}\left(\psi^{[h,-1]}\right)$ as in (4.23) and let us consider the terms contributing to $\overline{\mathcal{V}}_{a, 1}^{(-1)}\left(\psi^{[h,-1]}\right)$. The analysis goes exactly as before when no one or both the fields $\hat{\psi}_{\mathbf{k},-}^{+}$and $\hat{\psi}_{\mathbf{k}-\mathbf{p},-}^{-}$of $\delta \rho_{\mathbf{p},-}$ are contracted. This is not true if only one among the fields $\hat{\psi}_{\mathbf{k},-}^{+}$and $\hat{\psi}_{\mathbf{k}-\mathbf{p},-}^{-}$in $T_{2}(\psi)$ is contracted, since in this case there are marginal terms with two external lines, which before were absent. The terms with four external lines can be treated as before; one has just to substitute $D_{+}\left(\mathbf{k}^{-}\right) \hat{g}_{+}^{(0)}\left(\mathbf{k}^{+}\right)$with $D_{-}\left(\mathbf{k}^{-}\right) g_{-}^{(0)}\left(\mathbf{k}^{+}\right)$in the r.h.s. of (4.28), but this has no relevant consequence. The terms with two external lines have the form

$$
\begin{aligned}
& \int d \mathbf{k}^{-} \hat{\psi}_{\overline{\mathbf{k}}_{4},-}^{+} \hat{g}_{-}\left(\overline{\mathbf{k}}_{4}\right) \hat{J}_{\overline{\mathbf{k}}_{4}} \tilde{\chi}_{M}\left(\overline{\mathbf{k}}^{4}-\mathbf{k}^{-}\right) G_{1}^{(0)}\left(\mathbf{k}_{-}\right) \cdot \\
& \cdot\left\{\frac{\left[C_{h, 0}^{\varepsilon}\left(\overline{\mathbf{k}}_{4}\right)-1\right] D_{-}\left(\overline{\mathbf{k}}_{4}\right) \hat{g}_{-}^{(0)}\left(\mathbf{k}^{-}\right)}{D_{+}\left(\overline{\mathbf{k}}_{4}-\mathbf{k}^{-}\right)}-\frac{u_{0}\left(\mathbf{k}^{-}\right)}{D_{+}\left(\overline{\mathbf{k}}^{4}-\mathbf{k}^{-}\right)}\right\},
\end{aligned}
$$

where $G_{1}^{(0)}\left(\mathbf{k}_{-}\right)$is a smooth function of order 1 in $\lambda$. However, the first term in the braces is equal to 0 , since $\left|\overline{\mathbf{k}}_{4}\right|=\gamma^{h}$ implies that $C_{h, 0}^{\varepsilon}\left(\overline{\mathbf{k}}_{4}\right)-1=0$. Hence the r.h.s. of (4.83) is indeed of the form

$$
\int d \mathbf{k}^{-} \hat{\psi}_{\mathbf{k}_{4},-}^{+} \hat{g}_{-}\left(\overline{\mathbf{k}}_{4}\right) \hat{J}_{\overline{\mathbf{k}}_{4}} \tilde{\chi}_{M}\left(\overline{\mathbf{k}}_{4}-\mathbf{k}^{-}\right) G_{1}^{(0)}\left(\mathbf{k}_{-}\right) \frac{u_{0}\left(\mathbf{k}^{-}\right)}{D_{+}\left(\overline{\mathbf{k}}^{4}-\mathbf{k}^{-}\right)}
$$

so that it can be regularized in the usual way.

The analysis of $\overline{\mathcal{V}}_{a, 2}^{(-1)}\left(\psi^{[h,-1]}\right)$ can be done exactly as before. Hence, we can define again $\tilde{\lambda}_{-1}$ and $\tilde{z}_{-1}$ as in (4.30), with $\tilde{\lambda}_{-1}=O(\lambda)$ and $\tilde{z}_{-1}=O(1)$.

$11 /$ ottobre/2018; $1: 16$ 
Let us consider now the terms contributing to $\overline{\mathcal{V}}_{b, 1}^{(-1)}$, that is those where $\hat{\psi}_{\overline{\mathbf{k}}_{4}-\mathbf{p}}^{+}$is not contracted and there is a vertex of type $T_{2}$. Again the only marginal terms have four external lines and have the form

$$
\begin{aligned}
& \sum_{\tilde{\omega}} \int d \mathbf{p} \tilde{\chi}_{M}(\mathbf{p}) \hat{\psi}_{\mathbf{k}^{+}, \tilde{\omega}}^{+} \int d \mathbf{k}^{+} \hat{\psi}_{\mathbf{k}^{+-}-\mathbf{p}, \tilde{\omega}}^{+} \hat{\psi}_{\overline{\mathbf{k}}_{4}-\mathbf{p},-}^{+} \hat{g}_{-}\left(\overline{\mathbf{k}}_{4}\right) \hat{J}_{\overline{\mathbf{k}}_{4}} . \\
& \frac{D_{-}(\mathbf{p})}{D_{+}(\mathbf{p})}\left[F_{2,-, \tilde{\omega}}^{(-1)}\left(\mathbf{k}^{+}, \mathbf{k}^{+}-\mathbf{p}\right)+F_{1,-}^{(-1)}\left(\mathbf{k}^{+}, \mathbf{k}^{+}-\mathbf{p}\right) \delta_{-, \tilde{\omega}}\right],
\end{aligned}
$$

where we are using again the definition (132) of [BM2] (hence we have to introduce in the r.h.s. of (4.85) the factor $\left.D_{-}(\mathbf{p}) / D_{+}(\mathbf{p})\right)$. The analysis of the terms $F_{1,-}^{(-1)}\left(\mathbf{k}^{+}, \mathbf{k}^{+}-\mathbf{p}\right)$ is identical to the one in $\S 4.3$, while, as shown in [BM2], the symmetry property (4.35) implies now that, if we define

$$
F_{2,-, \tilde{\omega}}^{-1}\left(\mathbf{k}^{+}, \mathbf{k}^{-}\right)=\frac{1}{D_{-}(\mathbf{p})}\left[p_{0} A_{0,-\tilde{\omega}}\left(\mathbf{k}^{+}, \mathbf{k}^{-}\right)+p_{1} A_{1,-, \tilde{\omega}}\left(\mathbf{k}^{+}, \mathbf{k}^{-}\right)\right]
$$

and

$$
\mathcal{L} F_{2,-, \tilde{\omega}}^{-1}=\frac{1}{D_{-}(\mathbf{p})}\left[p_{0} A_{0,-, \tilde{\omega}}(0,0)+p_{1} A_{1,-, \tilde{\omega}}(0,0)\right]
$$

then

$$
\mathcal{L} F_{2,-,+}^{-1}=Z_{-1}^{3,-} \frac{D_{-}(\mathbf{p})}{D_{+}(\mathbf{p})} \quad, \quad \mathcal{L} F_{2,-,-}^{-1}=Z_{-1}^{3,+}
$$

where $Z_{-1}^{3,+}$ and $Z_{-1}^{3,-}$ are the same real constants appearing in (4.38). Hence, the local part of the marginal term (4.85) is, by definition, equal to

$$
Z_{-1}^{3,+} T_{+}\left(\psi^{[h,-1]}\right)+Z_{-1}^{3,-} T_{-}\left(\psi^{[h,-1]}\right) .
$$

The analysis of $\overline{\mathcal{V}}_{b, 2}^{(-1)}$ can be done exactly as before, so that we can write for $\overline{\mathcal{V}}^{(-1)}$ an expression similar to $(4.42)$, with $T_{2}\left(\psi^{[h,-1]}\right)$ in place of $T_{1}\left(\psi^{[h,-1]}\right)$ and $\nu_{-1, \pm}^{\prime}$ in place of $\nu_{-1, \pm}$. One can prove that, for simple symmetry reasons, $\nu_{-1, \pm}^{\prime}=\nu_{-1, \mp}$, if $\nu_{ \pm}^{\prime}=\nu_{\mp}$, but this property will not play any role, hence we will not prove it.

The integration of higher scales proceed as in $\S 4.4$. In fact, the only real difference we found in the integration of the first scale was in the calculation of the $O(1)$ terms contributing to $\tilde{z}_{-1}$, but these terms are absent in the case of $\tilde{z}_{j}, j \leq-2$, because the second term in the expression analogous to $(4.83)$, obtained by contracting on scale $j<0$ only one of the fields of $\delta \rho_{\mathbf{p},-}$, is exactly zero. It follows that the tree structure of the expansion is the same as that described in $\S 4.5$ and the constants $\nu_{\omega}^{\prime}$ can be chosen again so that the bound (4.44) is satisfied even by the constants $\nu_{j, \omega}^{\prime}$. In the analysis of the constants $\tilde{\lambda}_{j}$ and $\tilde{z}_{j}$ there is only one difference, concerning the bound (4.72), which has to be substituted with $\tilde{z}_{-1}-\alpha z_{-1} \leq C$, in the case $j=-1$, but it is easy to see that this has no effect on the bound (4.77). It follows that the final considerations of $\S 4.8$ stay unchanged and we get for $\tilde{G}_{-}^{4}\left(\mathbf{k}_{1}, \mathbf{k}_{2}, \mathbf{k}_{3}, \mathbf{k}_{4}\right)$ a bound similar to that proved for $\tilde{G}_{+}^{4}\left(\mathbf{k}_{1}, \mathbf{k}_{2}, \mathbf{k}_{3}, \mathbf{k}_{4}\right)$, so ending the proof of Lemma 3.1 .

\section{Appendix A1. The ultraviolet problem and the Thirring model}

Thanks to the linearity of the propagator, the above analysis can be used with no essential modifications to construct the massless Thirring model (see for instance [Z]), by removing the ultraviolet cutoff. We shall sketch here the main ideas; the details will be published elsewhere.

The Thirring model describes Dirac fermions in $d=1+1$ interacting with a local current-current interaction; its action is

$$
\int d \mathbf{x}\left[-\bar{\psi}_{\mathbf{x}} \not \partial \psi_{\mathbf{x}}-\frac{\lambda}{4} J_{\mu}(\mathbf{x}) J^{\mu}(\mathbf{x})\right]
$$


where $\not \partial=\gamma_{0} \partial_{x_{0}}+\gamma_{1} \partial_{x}, \mathbf{x}=\left(x_{0}, x\right), \bar{\psi}_{\mathbf{x}}=\psi_{\mathbf{x}}^{+} \gamma_{0}, \psi_{\mathbf{x}}$ is a two component spinor field (to not be confused with a Grassmannian field), $J_{\mu}(\mathbf{x})=\bar{\psi}_{\mathbf{x}} \gamma_{\mu} \psi_{\mathbf{x}}$ and $\gamma_{0}=\sigma_{1}, \gamma_{1}=\sigma_{2}$ are Pauli matrices.

The generating functional of the Thirring model is the following Grassmannian integral with infrared cutoff $\gamma^{h}$ and ultraviolet cutoff $\gamma^{N}$, with $h, N$ integers and $N>0$

$$
\begin{aligned}
& \mathcal{W}(\phi, J)=\log \int P_{Z_{N}}\left(d \psi^{[h, N]}\right) \exp \left\{-V\left(\psi^{[h, N]}\right)+\right. \\
& \left.+\sum_{\omega} \int d \mathbf{x}\left[Z_{N}^{(2)} J_{\mathbf{x}, \omega} \psi_{\mathbf{x}, \omega}^{[h, N]+} \psi_{\mathbf{x}, \omega}^{[h, N]-}+\phi_{\mathbf{x}, \omega}^{+} \psi_{\mathbf{x}, \omega}^{[h, N]-}+\psi_{\mathbf{x}, \omega}^{[h, N]+} \phi_{\mathbf{x}, \omega}^{[h, N]-}\right]\right\},
\end{aligned}
$$

where $P_{Z_{N}}\left(d \psi^{[h, N]}\right)$ is given by $(1.3)$, with $C_{h, 0}(\mathbf{k})$ replaced by $C_{h, N}(\mathbf{k})=\sum_{j=h}^{N} f_{j}(\mathbf{k})$ and $\psi_{\mathbf{k}, \omega}^{[h, 0] \sigma}$ replaced by $\sqrt{Z_{N}} \psi_{\mathbf{k}, \omega}^{[h, N] \sigma}$, and $V\left(\psi^{[h, N]}\right)$ is given by

$$
V\left(\psi^{[h, N]}\right)=\tilde{\lambda}_{N} \int d \mathbf{x} \psi_{\mathbf{x},+}^{[h, N]+} \psi_{\mathbf{x},+}^{[h, N]-} \psi_{\mathbf{x},-}^{[h, N]+} \psi_{\mathbf{x},-}^{[h, N]-} ;
$$

$Z_{N}$ is the (bare )wave function renormalization, $Z_{N}^{(2)}$ is the (bare )density renormalization and $\tilde{\lambda}_{N}$ is the (bare) interaction. In order to get a nontrivial limit as $N \rightarrow \infty$, it is convenient to write $\tilde{\lambda}_{N}$ and $Z_{N}^{(2)}$ in terms of $Z_{N}$ and two new bare constants, $\lambda_{N}$ and $c_{N}$, in the following way:

$$
\tilde{\lambda}_{N}=\left(Z_{N}\right)^{2} \lambda_{N} \quad, \quad Z_{N}^{(2)}=c_{N} Z_{N}
$$

One expects that the model is well defined if $\lambda_{N}$ and $c_{N}$ converge to finite non zero limits and $Z_{N} \rightarrow 0$, as $N \rightarrow \infty$; moreover, in order to apply our perturbative procedure, $\lambda_{N}$ has to be small enough, uniformly in $N$.

The proof of this claim is essentially a corollary of the above analysis for the infrared problem. The RG analysis in $\S 2$ can be repeated by allowing the scale index $j$ to be positive or negative. The Ward identity (3.14) holds with a factor $Z_{N} / Z_{N}^{(2)}$ multiplying $G_{+}^{2,1}$ and $\Delta_{+}^{2,1}$, and we get the identity

$$
\left|\frac{Z_{h}^{(2)}}{c_{N} Z_{h}}-1\right| \leq C \bar{\lambda}_{h}^{2} .
$$

In the same way, from the Dyson equation (identical to (1.11), with $\lambda_{N} Z_{N} / Z_{N}^{(2)}$ in place of $\lambda$ in the r.h.s), and proceeding as in $\S 3$ and $\S 4$, we get that for any $h$ one has $\left|\lambda_{h}-\lambda_{N}\right| \leq c_{3} \bar{\lambda}_{h+1}^{2}$ (compare with (3.2)), so that the expansion is convergent, if $\lambda_{N}$ is small enough. By (3.4), $Z_{N}$ must be chosen so that $Z_{N} \gamma^{N \eta\left(\lambda_{N}\right)}$ is convergent for $N \rightarrow \infty$ to some constant, which can be fixed by requiring, for instance, that $Z_{0}=1$. In the same way we can fix $\lim _{N \rightarrow \infty} \lambda_{N}$ so that, for instance, $\lambda_{0}=\lambda$, with $\lambda$ small enough; of course $\lambda_{N}=\lambda+O\left(\lambda^{2}\right)$. The choice of $\lim _{N \rightarrow \infty} c_{N}$ is a free parameter, whose value has no special role.

Finally we shall discuss the form taken from Ward identities when the ultraviolet and the infrared cutoff are removed. The analogous of (3.20) for the model (A1.2) is

$$
\begin{aligned}
& \frac{Z_{N}}{Z_{N}^{(2)}}\left(1-\nu_{+}\right) D_{+}(\mathbf{p}) \hat{G}_{+}^{4,1}\left(\mathbf{p}, \mathbf{k}_{1}, \mathbf{k}_{2}, \mathbf{k}_{3}, \mathbf{k}_{4}-\mathbf{p}\right)-\frac{Z_{N}}{Z_{N}^{(2)}} \nu_{-} D_{-}(\mathbf{p}) \hat{G}_{-}^{4,1}\left(\mathbf{p}, \mathbf{k}_{1}, \mathbf{k}_{2}, \mathbf{k}_{3}, \mathbf{k}_{4}-\mathbf{p}\right)(A 1.6) \\
& =\hat{G}_{+}^{4}\left(\mathbf{k}_{1}-\mathbf{p}, \mathbf{k}_{2}, \mathbf{k}_{3}, \mathbf{k}_{4}-\mathbf{p}\right)-\hat{G}_{+}^{4}\left(\mathbf{k}_{1}, \mathbf{k}_{2}+\mathbf{p}, \mathbf{k}_{3}, \mathbf{k}_{4}-\mathbf{p}\right)+Z_{N} H_{+}^{4,1}\left(\mathbf{p}, \mathbf{k}_{1}, \mathbf{k}_{2}, \mathbf{k}_{3}, \mathbf{k}_{4}-\mathbf{p}\right)
\end{aligned}
$$

with $H_{+}^{4,1}$ given by (3.21) with the cutoff function $C_{h, 0}^{-1}$ replaced by $C_{h, N}^{-1}$. The counterterms $\nu_{ \pm}$are found by a fixed point method as in Lemma 4.1, with the only difference that the ultraviolet scale 0 is replaced by the scale $N ; \nu_{ \pm}, \nu_{ \pm}^{\prime}$ tend to a non vanishing well defined limit as $N \rightarrow \infty, h \rightarrow-\infty$. 
In the same way the analogous of $(3.22)$ is

$$
\begin{aligned}
& \frac{Z_{N}}{Z_{N}^{(2)}}\left(1-\nu_{-}^{\prime}\right) D_{-}(\mathbf{p}) \hat{G}_{-}^{4,1}\left(\mathbf{p}, \mathbf{k}_{1}, \mathbf{k}_{2}, \mathbf{k}_{3}, \mathbf{k}_{4}-\mathbf{p}\right)-\frac{Z_{N}}{Z_{N}^{(2)}} \nu_{+}^{\prime} D_{+}(\mathbf{p}) \hat{G}_{+}^{4,1}\left(\mathbf{p}, \mathbf{k}_{1}, \mathbf{k}_{2}, \mathbf{k}_{3}, \mathbf{k}_{4}-\mathbf{p}\right) \\
& =\hat{G}_{+}^{4}\left(\mathbf{k}_{1}, \mathbf{k}_{2}, \mathbf{k}_{3}-\mathbf{p}, \mathbf{k}_{4}-\mathbf{p}\right)-\hat{G}_{+}^{4}\left(\mathbf{k}_{1}, \mathbf{k}_{2}, \mathbf{k}_{3}, \mathbf{k}_{4}\right)+Z_{N} H_{-}^{4,1}\left(\mathbf{p}, \mathbf{k}_{1}, \mathbf{k}_{2}, \mathbf{k}_{3}, \mathbf{k}_{4}-\mathbf{p}\right),
\end{aligned}
$$

It is a straightforward consequence of our analysis (in particular of the short memory property we used extensively throughout the paper) that in (3.20) and (3.22) for the model (A1.2), if the external momenta have a fixed (i.e. independent from $h, N)$ value, then

$$
\lim _{\substack{N \rightarrow \infty \\ h \rightarrow-\infty}} Z_{N} \hat{H}_{ \pm}^{4,1}\left(\mathbf{p}, \mathbf{k}_{1}, \mathbf{k}_{2}, \mathbf{k}_{3}, \mathbf{k}_{4}-\mathbf{p}\right)=0 .
$$

Hence, if we sum the two Ward identities above and remember that $\nu_{+}=\nu_{-}^{\prime}$ and $\nu_{-}=\nu_{+}^{\prime}$, we get, in the limit $N \rightarrow \infty, h \rightarrow-\infty$

$$
\begin{aligned}
& \sum_{\omega= \pm} D_{\omega}(\mathbf{p}) \frac{1-\nu_{+}-\nu_{-}^{\prime}}{c_{N}} \hat{G}_{\omega}^{4,1}\left(\mathbf{p}, \mathbf{k}_{1}, \mathbf{k}_{2}, \mathbf{k}_{3}, \mathbf{k}_{4}-\mathbf{p}\right)=G_{+}^{4}\left(\mathbf{k}_{1}-\mathbf{p}, \mathbf{k}_{2}, \mathbf{k}_{3}, \mathbf{k}_{4}-\mathbf{p}\right)- \\
& -\hat{G}_{+}^{4}\left(\mathbf{k}_{1}, \mathbf{k}_{2}+\mathbf{p}, \mathbf{k}_{3}, \mathbf{k}_{4}-\mathbf{p}\right)+\hat{G}_{+}^{4}\left(\mathbf{k}_{1}, \mathbf{k}_{2}, \mathbf{k}_{3}-\mathbf{p}, \mathbf{k}_{4}-\mathbf{p}\right)-\hat{G}_{+}^{4}\left(\mathbf{k}_{1}, \mathbf{k}_{2}, \mathbf{k}_{3}, \mathbf{k}_{4}\right)
\end{aligned}
$$

The above Ward identity is identical to the formal one, obtained by a total gauge transformation, except for the factor $\left(1-\nu_{+}-\nu_{-}^{\prime}\right) / c_{N}$ multiplying $G^{4,1}$; in other words, the formal Ward identity holds when the cutoffs are removed, up to a finite interaction-dependent renormalization of the density operator. A similar phenomenon appears also in perturbative QED $[\mathrm{H}]$ and is called soft breaking of gauge invariance. Of course it is possible to choose $Z_{N}^{(2)}$ so that the formal Ward identity is verified, i.e. we can choose $c_{N}=\left(1-\nu_{+}-\nu_{-}^{\prime}\right)$.

On the contrary, the Ward identities (3.20) and (3.22), obtained by a chiral gauge transformation, do not tend in the limit to the formal Ward identities (obtained by (3.20) and (3.22) putting $\left.\nu_{ \pm}=\nu_{ \pm}^{\prime}=0\right)$; beside the renormalization of the density operator, an extra factor appears in the identity, namely $G_{-}^{4,1}$ in $(3.20)$ or $G_{+}^{4,1}$ in (3.22). This phenomenon is called chiral anomaly, see $[\mathrm{Z}]$, and is is present also in perturbative QED.

\section{References}

[A] P.W. Anderson. The theory superconductivity in high $T_{c}$ cuprates, Princeton University Press (1997)

[Af] I. Affleck: Field theory methods and quantum critical phenomena. Proc. of Les Houches summer school on Critical phenomena, Random Systems, Gauge theories, North Holland (1984).

[BG] G. Benfatto, G. Gallavotti: Perturbation Theory of the Fermi Surface in a Quantum Liquid. A General Quasiparticle Formalism and One-Dimensional Systems. J. Stat. Phys. 59, 541-664 (1990).

[BGPS] G. Benfatto, G. Gallavotti, A. Procacci, B. Scoppola: Beta Functions and Schwinger Functions for a Many Fermions System in One Dimension. Comm. Math. Phys. 160, 93-171 (1994).

[BM1] G. Benfatto, V. Mastropietro: Renormalization Group, hidden symmetries and approximate Ward identities in the XYZ model. Rev. Math. Phys. 13, 1323-1435 (2001).

[BM2] G. Benfatto, V. Mastropietro: On the density-density critical indices in interacting Fermi systems. Comm. Math. Phys. 231, 97-134 (2002).

[BM3] G. Benfatto, V. Mastropietro: Ward identities and vanishing of the Beta function for $d=1$ interacting Fermi systems. J. Stat. Phys. 115, 143-184 (2004).

[BoM] F. Bonetto, V. Mastropietro: Beta Function and Anomaly of the Fermi Surface for a $d=1$ System of Interacting Fermions in a Periodic Potential. Comm. Math. Phys. 172, 57-93 (1995). 
[DL] I.E. Dzyaloshinky, A.I. Larkin: Correlation functions for a one-dimensional Fermi system with long-range interaction (Tomonaga model). Soviet Phys. JETP 38, 202-208 (1974).

[G] T. Giamarchi.: Quantum Physics in one dimension. International Series of Monographs on Physics 121, Clarendon Press, Oxford (2004).

[GM] G. Gentile, V. Mastropietro: Renormalization Group for fermions: a review of mathematical results. Phys. Rep. 352, 273-437 (2001).

[H] T.R. Hurd: Soft breaking of Gauge invariance in regularized Quantum Electrodynamics. Comm. Math. Phys 125, 515-526 (1989).

[Le] A. Lesniewski: Effective action for the Yukawa 2 quantum field Theory. Commun. Math. Phys. 108, 437-467 (1987).

[M] V.Mastropietro, Small denominators and anomalous behaviour in the incommensurate Hubbard-Holstein model.Comm. Math. Phys. 201, 81-115 (1999)

[M1] V.Mastropietro, Coupled Ising models with quartic interaction at criticality, Comm. Math. Phys 244, 595-642 (2004)

[MD] W. Metzner, C. Di Castro:Conservation laws and correlation functions in the Luttinger liquid. Phys. Rev. B 47, 16107 (1993).

[ML] D. Mattis, E. Lieb: Exact solution of a many fermion system and its associated boson field. J. Math. Phys. 6, 304-312 (1965).

[S] J. Solyom: The Fermi gas model of one-dimensional conductors. Adv. in. Phys. 28, 201-303 (1979).

[T] W. Thirring: Ann. of Phys. 3, 91 (1958).

[Z] J. Zinn-Justin: Quantum field theory and critical phenomena, Oxford publications, (1989). 